Florida International University FIU Digital Commons

3-21-2012

\title{
Influence of Religious Coping on the Substance Use and HIV Risk Behaviors of Recent Latino Immigrants
}

Mariana Sanchez

Florida International University, msanc062@fiu.edu

DOI: $10.25148 /$ etd.FI12050209

Follow this and additional works at: https:// digitalcommons.fiu.edu/etd

\section{Recommended Citation}

Sanchez, Mariana, "Influence of Religious Coping on the Substance Use and HIV Risk Behaviors of Recent Latino Immigrants" (2012). FIU Electronic Theses and Dissertations. 603.

https://digitalcommons.fiu.edu/etd/603 


\section{FLORIDA INTERNATIONAL UNIVERSITY}

Miami, Florida

INFLUENCE OF RELIGIOUS COPING ON THE SUBSTANCE USE AND HIV RISK

BEHAVIORS OF RECENT LATINO IMMIGRANTS

A dissertation submitted in partial fulfillment of

the requirements for the degree of

DOCTOR OF PHILOSOPHY

in

SOCIAL WELFARE

by

Mariana Sanchez 
To: Interim Dean Michele Ciccazzo

R.Stempel College of Public Health and Social Work

This dissertation, written by Mariana Sanchez, and entitled Influence of Religious Coping on the Substance Use and HIV Risk Behaviors of Recent Latino Immigrants, having been approved in respect to style and intellectual content, is referred to you for judgment.

We have read this dissertation and recommend that it be approved.

Eric Wagner

Mark Macgowan

Frank Dillon

Elena Bastida

Mario De La Rosa, Major Professor

Date of Defense: March 21, 2012

The dissertation of Mariana Sanchez is approved.

Interim Dean Michele Ciccazzo

R.Stempel College of Public Health and Social Work

Dean Lakshmi N. Reddi

University Graduate School

Florida International University, 2012 


\section{DEDICATION}

This dissertation is dedicated to my family. To Amauri, my best friend, my life partner, my world. You are my pillar of strength. Your unwavering guidance and support makes this achievement just as much yours as it is mine. Without you none of this would have been possible. I love you today, tomorrow, and forever. To my mother, who has dedicated her life to being an amazing mom. Your unconditional love, support, and all the sacrifices you endured has made me who I am today. I love you Mami. To my grandmother, for her incredible strength and resiliency. And finally, to my daughter Emily who has given me a new perspective on life. I never knew just how much I could love someone until you came along. 


\section{ACKNOWLEDGMENTS}

I would like to first and foremost acknowledge my Major Professor, Mario De La Rosa, to whom I am continually grateful. Your guidance, support, and mentorship has lead me to the place I am today. Thank you to my committee members, Dr. Mark Macgowan, Dr. Eric Wagner, and Dr. Elena Bastida for their constructive feedback and contributions to my learning process. I would like to extend my particular gratitude to Dr. Frank Dillon for his many hours of guidance, skilled instruction, and assistance in shaping this project into one of which I can be proud of.

I'd like to acknowledge the CRUSADA staff for all of their hard work and dedication in collecting and entering the data that was used for this project. I would like to particularly acknowledge Samuel Martinez whose technical expertise was pivotal in putting this dissertation together.

I would also like to thank all of my friends for their time, effort, and support on this journey. Adi, for always being my sounding board and voice of reason in my times of despair. To Beverly, my partner in crime, thank you for sticking by me every step of the way. Jackie, for being so wonderfully patient and understanding with my lack of time and attention. I'd like to acknowledge my fellow doctoral students, and in particular Julieta, Sheila, and Bora for their kindness, encouragement, and support. You have all contributed in your own way to make this a reality.

Finally, thank you to the National Institute on Drug Abuse for their financial contributions to my doctoral studies and professional development. 


\title{
ABSTRACT OF THE DISSERTATION \\ INFLUENCE OF RELIGIOUS COPING ON THE SUBSTANCE USE AND HIV RISK \\ BEHAVIORS OF RECENT LATINO IMMIGRANTS
}

\author{
by \\ Mariana Sanchez \\ Florida International University, 2012 \\ Miami, Florida \\ Professor Mario De La Rosa, Major Professor
}

This study examines the influence of acculturative stress on substance use and HIV risk behaviors among recent Latino immigrants. The central hypothesis of the study is that specific religious coping mechanisms influence the relationship that acculturative stress has on the substance use and HIV-risk behaviors of recent Latino immigrants. Within the Latino culture religiosity is a pervasive force, guiding attitudes, behaviors, and even social interactions. When controlling for education and socioeconomic status, Latinos have been found to use religious coping mechanisms more frequently than their NonLatino White counterparts. In addition, less acculturated Latinos use religious coping strategies more frequently than those with higher levels of acculturation. Given its prominent role in Latino culture, it appears probable that this mechanism may prove to be influential during difficult life transitions, such as those experienced during the immigration process. This study examines the moderating influence of specific religious coping mechanisms on the relationship between acculturative stress and substance use/HIV risk behaviors of recent Latino immigrants. Analyses for the present study were conducted with wave 2 data from an ongoing longitudinal study investigating 
associations between pre-immigration factors and health behavior trajectories of recent Latino immigrants. Structural equation and zero-inflated Poisson modeling were implemented to test the specified models and examine the nature of the relationship among the variables. Moderating effects were found for negative religious coping. Higher levels of negative religious coping strengthened an inverse relationship between acculturative stress and substance use. Results also indicated direct relationships between religious coping mechanisms and substance use. External and positive religious coping were inversely related to substance use. Negative religious coping was positively related to substance use. This study aims to contribute knowledge of how religious coping influence's the adaptation process of recent Latino immigrants. Expanding scientific understanding as to the function and effect of these coping mechanisms could lead to enhanced culturally relevant approaches in service delivery among Latino populations. Furthermore this knowledge could inform research about specific cognitions and behaviors that need to be targeted in prevention and treatment programs with this population. 


\section{TABLE OF CONTENTS}

CHAPTER

PAGE

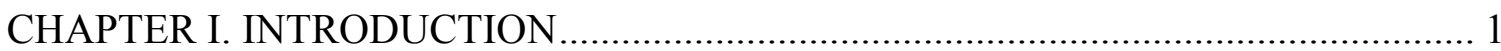

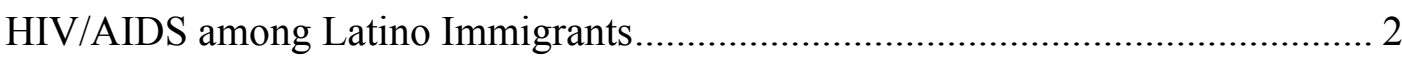

HIV/AIDS in South Florida ........................................................................... 3

Substance Use among Latinos......................................................................... 4

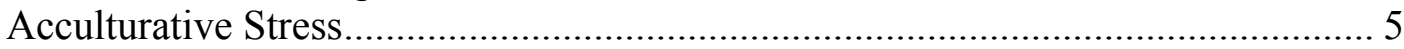

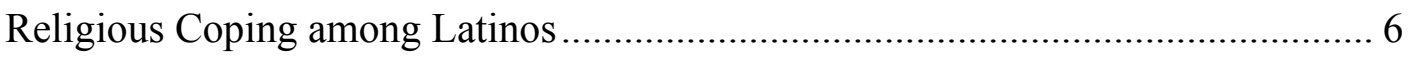

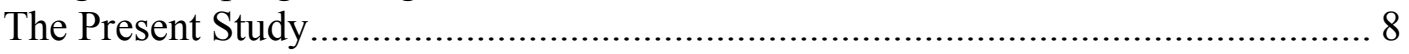

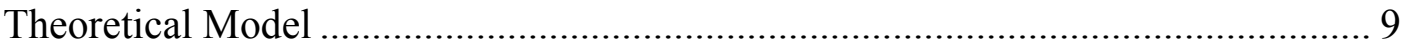

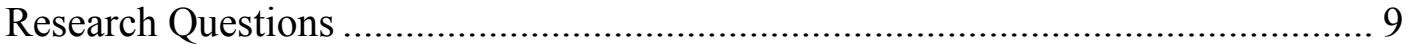

CHAPTER II. REVIEW OF RELEVANT CONSTRUCTS .......................................... 12

Religion in the Latino Culture......................................................................... 12

The Religion-Health Connection .......................................................................... 15

Religion and HIV-Risk Behaviors ................................................................... 16

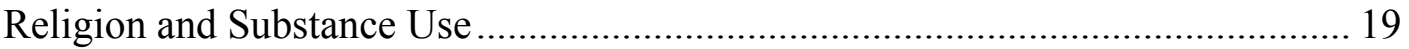

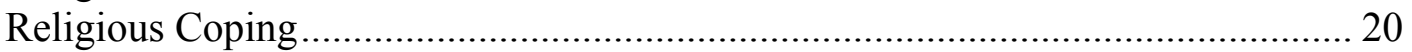

Latinos and religious coping. ............................................................................ 22

Conceptual models of religious coping. ............................................................. 24

Positive and negative religious coping. ........................................................... 27

Internal and external religious coping............................................................ 29

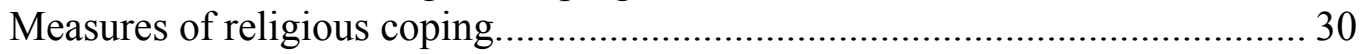

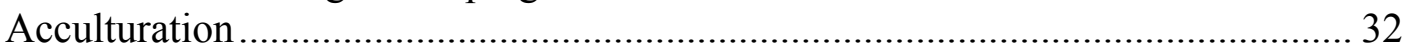

Conceptual models of acculturation. .................................................................. 33

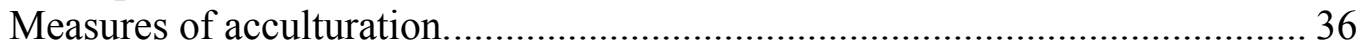

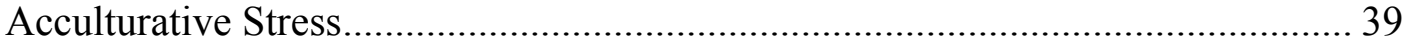

Conceptual model of acculturative stress......................................................... 42

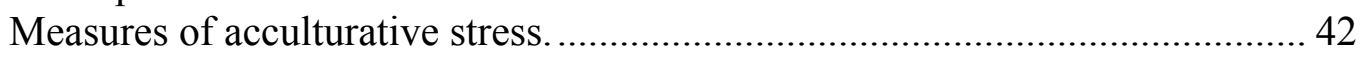

Acculturation and HIV Risk Behaviors among Latinos........................................... 43

Acculturation and Substance Use among Latinos.................................................... 45

Acculturative Stress and Religious Coping ....................................................... 50

Summary of Current Study ............................................................................... 51

Conceptual Framework for Current Study ……………......................................... 52

Research Aims and Hypotheses ......................................................................... 53

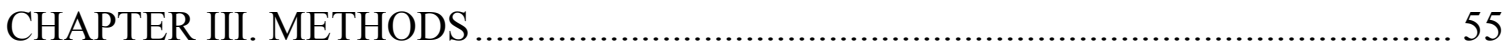

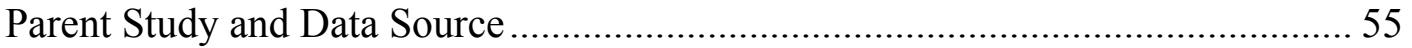

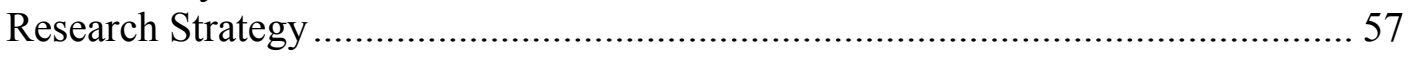

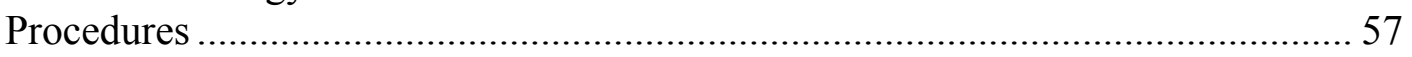

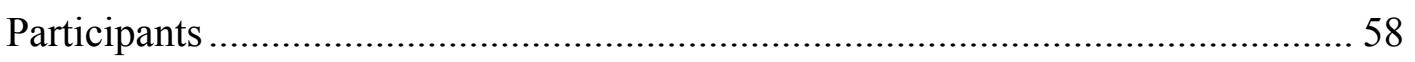

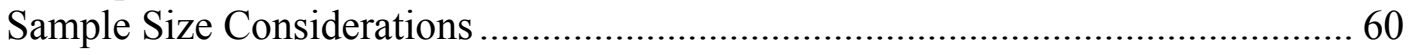

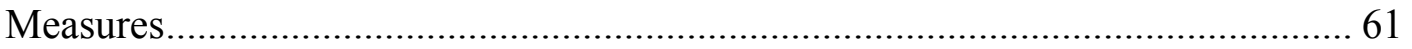

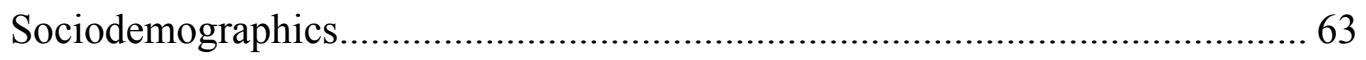




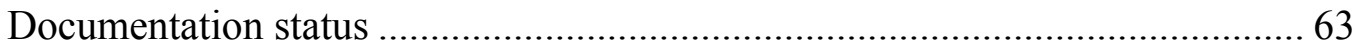

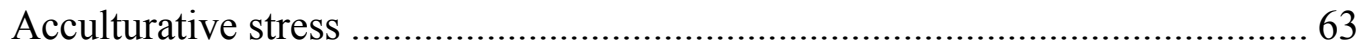

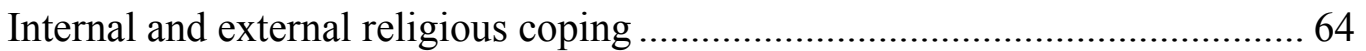

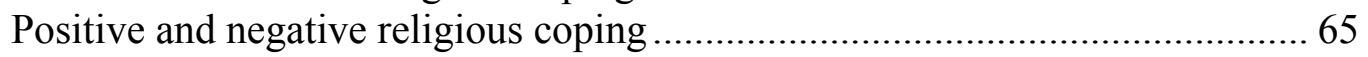

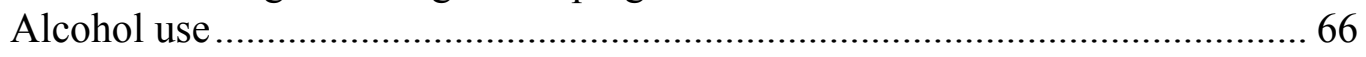

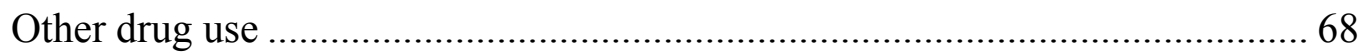

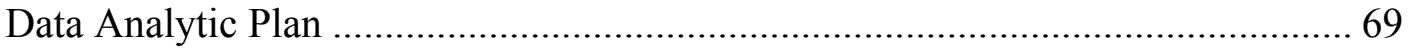

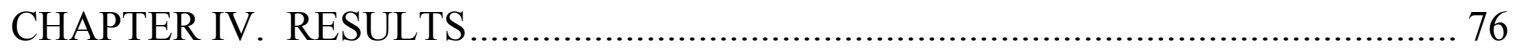

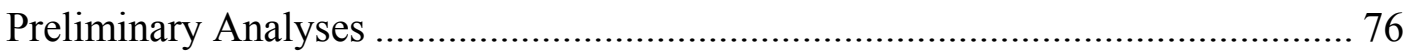

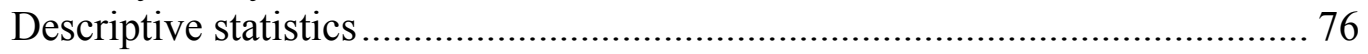

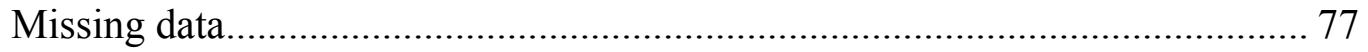

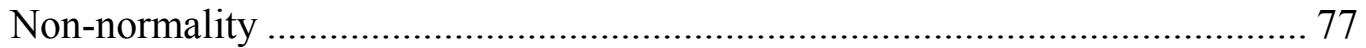

Correlational analyses ....................................................................... 78

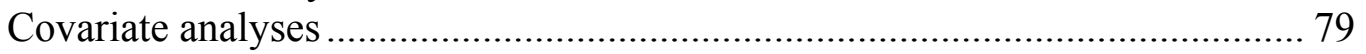

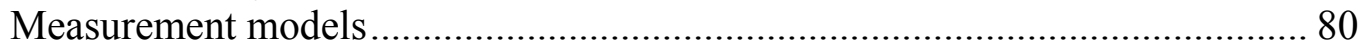

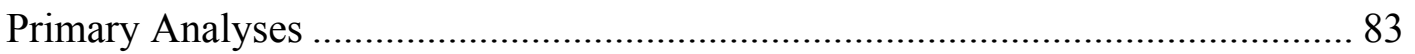

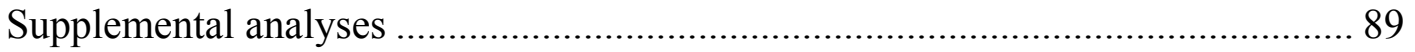

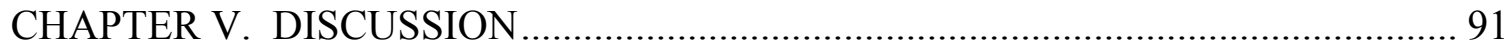

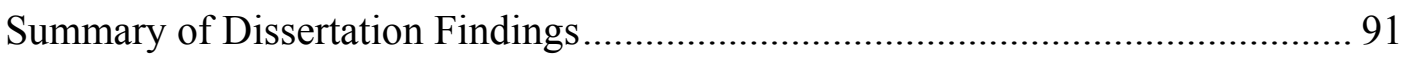

Associations between negative religious coping and substance use. ................ 92

Associations between external/positive religious coping and substance use. .... 94

Acculturative stress and religious coping .................................................. 95

Associations between documentation status and drug use behaviors. .............. 96

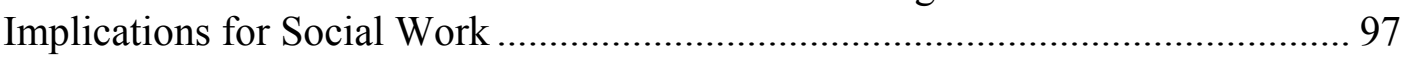

Micro-level social work practice implications ........................................... 97

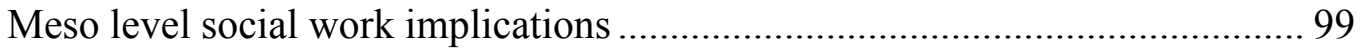

Macro level social work implications.............................................................. 100

Future Directions for Research, Intervention, and Policy ................................ 104

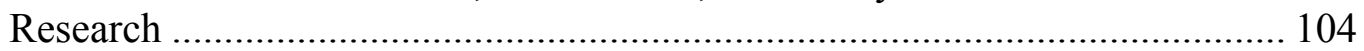

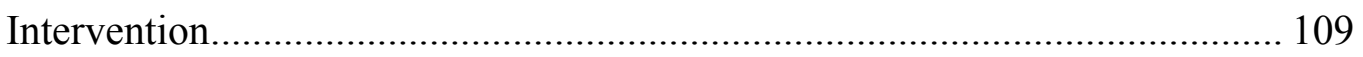

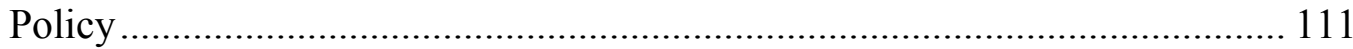

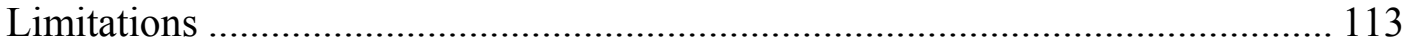

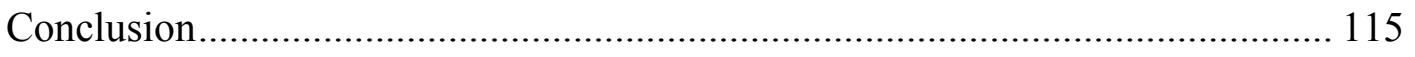

LIST OF REFERENCES .............................................................................. 134

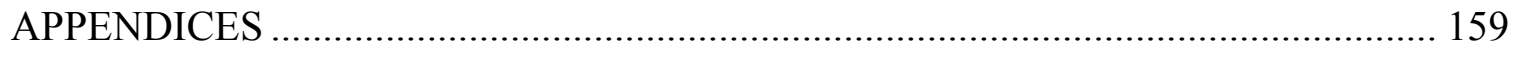

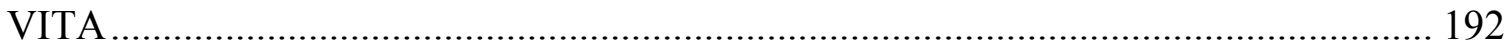




\section{LIST OF TABLES}

TABLE

PAGE

1. Framework of Contextual Factors Influencing Acculturation

2. Dimensions of Acculturative Stress

3. Inclusion and Exclusion Criteria for Parent Study

4. Descriptive Characteristics of Sample $(n=415)$

118

5. Frequencies for Key Observed Variables

120

6. Correlations of Key Variables

121 


\section{LIST OF FIGURES}

FIGURE

PAGE

1. Measurement Model for Pre-immigration Alcohol Use

2. Measurement Model for Post-immigration Alcohol Use

3. Measurement Model for Pre-immigration HIV Risk

4. Measurement Model for Post-immigration HIV Risk

5. Structural Model Testing Alcohol Use and HIV Risk Outcomes

126

6. Zero-inflated Poissson Model Testing Drug Use Outcomes

7. Results of Structural Model Testing Alcohol Use and HIV Risk Outcomes

8. Results of Zero-inflated Poissson Model Testing Drug Use Outcomes

9. Moderation Effect of Negative Religious Coping on Relationship between Acculturative Stress and Alcohol Use

10. Moderation Effect of Negative Religious Coping on Relationship Between Acculturative Stress and Drug Use Frequency

11. Supplemental Analysis Testing Direct Effects of Religious Coping Mechanisms on Alcohol Use and HIV Risk Behaviors

12. Supplemental Analysis Testing Direct Effects of Religious Coping Mechanisms Testing Direct Effects of Religious Coping Mechanisms on Drug Use 


\section{CHAPTER I. INTRODUCTION}

The Latino population in the United States has experienced remarkably rapid growth over the last three decades. Statistics from the 2010 Census placed Latinos at approximately 50.5 million people, accounting for $16 \%$ of the total U.S. population. More than half of the growth in the total U.S. population between 2000 and 2010 was due to the increase in the Latino population. Specifically, within the last decade the number of Latinos in the U.S. grew by $43 \%$, which was four times the growth in the total population at 10 percent (U.S. Census, 2011). A distinctive characteristic of the Latino population in the United States is its large number of immigrants. Today, the majority of immigrants gaining entry into the United States are Latino (Caplan, 2007). It is anticipated that the population of the United States will rise to 438 million in 2050, from 296 million in 2005 , and $82 \%$ of the increase will be due to immigrants arriving from 2005 to 2050 . The Latino population, already the largest minority group, is expected to triple in size and account for most of the nation's population growth from 2005 to 2050 . By the year 2025, the immigrant, or foreign born, share of the population is projected to surpass the peak during the last great wave of immigration a century ago (Pew Hispanic Center, 2009).

Escalating rates of immigration into the United States has led to nation with an increasingly diverse population. However, while diversity of the American population is one of the Nation's greatest assets, the health status of racial and ethnic minorities has becomes one of its greatest challenges (National Institute of Minority Health and Health Disparities, 2009). The pattern of health disparities among Latino immigrants tends to be particularly confounding in nature. Regardless of whether these individuals come to the 
U.S. for economic, political, or other reasons, all arrive in search of a better life. Yet, the longer these individuals live in the United States, the worse their health status becomes (Caplan, 2007). These findings have left researchers with the "immigrant paradox", a complex and confounding picture of Latino immigrant health, and a question to which the answer could have vast implications towards diminishing health disparities in the United States (Caplan, 2007).

\section{HIV/AIDS among Latino Immigrants}

As demographers have observed the rapid growth of the U.S. Latino immigrant population, researchers also determined that Latinos are disproportionally affected by the HIV/AIDS epidemic. Investigations into the underlying risk factors responsible for the spread of HIV/AIDS among Latino immigrant have attributed their increased rates of infection to sexual and substance use practices. These behaviors are often mediated by level of acculturation to U.S. society (Hines \& Caetano, 1998; Levy, et al., 2005). For example, as Latinas become more acculturated to American society, their substance use patterns and levels of sexual promiscuity tend to increase (Nyamathi, Bennet, Leake, Lewis, \& Flaskerud, 1993). This rise in risk behaviors are thought to be partially a result of an erosion of culturally protective values. Moreover, recently arrived Latinos are of particular concern as research indicates that less acculturated Latinos report a) less perceived risk of HIV/AIDS, b) less accurate knowledge of HIV/AIDS, c) are less likely to know where HIV testing can be obtained, and d) have higher stigma associated with HIV/AIDS (Shedlin, Ulises, \& Oliver-Velez, 2005).

While Latinos are the fastest growing ethnic minority population in the United States they are also the group with the second-highest risk for HIV. As of 2009, Latinos 
made up $20 \%$ of new HIV infections in the United States while representing approximately $16 \%$ of the total US population (CDC, 2011). Latino men accounted for $79 \%(7,400)$ of new infections among all Latinos in 2009. Nationally, the rate of new infections among Latino men is two and a half times as high as that of white men (39.9/100,000 vs. $15.9 / 100,000)$. The disparity is even more remarkable among Latina women who accounted for $21 \%(2,000)$ of new infections among Latinos in 2009. This rate is four and a half times higher that of their white counterparts $(11.8 / 100,000 \mathrm{vs.}$ 2.6/100,000) (CDC, 2011).

\section{HIV/AIDS in South Florida}

Since the beginning of the HIV epidemic in the early 1980s, the state of Florida and Miami-Dade County, the most populous county in Florida, have been at the epicenter, ranking consistently as one of the geographic areas with the highest HIV rates. As of 2010 there were 95,335 living with a diagnosis of HIV infection in the state of Florida. Moreover, Florida currently ranks third in the nation for highest number of cumulative reported AIDS cases (CDC, 2010).

Latinos in Florida appear to be disproportionately affected by the HIV epidemic. The proportion of all newly reported adult HIV cases among Latinos increased from 17\% in 2001 to $22 \%$ in 2010 (Florida Department of Health, 2011). Among newly reported adult HIV cases in 2010, the case rate among Latino men was 2 times higher than in white men and the case rate among Latina women was 4 times higher than in white women (Florida Department of Health, 2011). In 2009, Latino males accounted for 22\% of the HIV cases among men, while Latina females accounted for $13 \%$ of the total HIV cases among women in Florida (Florida Department of Health, 2009). HIV continues to 
particularly impact Latino young adults. In 2010 HIV was $6^{\text {th }}$ leading cause of death in Florida for Latino men ages 25-44; and the $5^{\text {th }}$ leading cause of death for Latina women for this age group (Florida Department of Health, 2009). Moreover, approximately 70\% of the state's 18,727 Latinos living with a diagnosis of HIV through 2010 were foreign born (Florida Department of Health, 2011).

Miami-Dade County has consistently ranked as the county with the highest number of HIV/AIDS cases in the state of Florida. Latinos currently make up $64 \%$ of the Miami-Dade County population. As of 2010 the county has an estimated 24,782 persons living with a diagnosis of HIV infection. Latinos make up 39\% of those cases (MiamiDade County Health Department, 2011). Latino males account for $46 \%$ of the total HIV case among men in Miami-Dade County, while Latina females account for 22\% (MiamiDade County Health Department, 2011). Similar to national disparities, Latinos are disproportionately affected by the HIV epidemic in Miami Dade County. As of 2010, among adult Latinos living in Miami Dade County (age 13+) one in 92 Latino men and one in 511 Latina women were living with HIV/AIDS, compared to one in 70 white men and 537 white women. Among Latinos living with HIV/AIDS in Miami-Dade County approximately 80\% are foreign-born (Miami-Dade County Health Department, 2011).

\section{Substance Use among Latinos}

There is vast literature indicating how the use alcohol and other drugs places individuals at risk for unsafe sexual practices (NIDA, 2006). Specifically, intoxication increases the likelihood of impulsive and risky decisions and decreases inhibition. Among persons aged 12 or older, Latinos have a 10\% rate of substance dependence or 
abuse. This is a higher rate than Whites (9.2\%) and African Americans (9\%) (NIDA, 2009).

Additionally, researchers have explored the role that stress associated with the acculturation process to American society has upon drug using behaviors of Latinos. Both family and personal stress factors associated with acculturation have been linked to substance use (Vega, Alderete, Kolody, \& Aguilar Gaxiola, 1998). Investigations into the substance abuse and acculturation patterns of Latinos indicate vast discrepancies in the drug use patterns that exist between acculturated and non-acculturated Latinos, with acculturated Latinos being 13 times more likely to report using illicit substances as their non-acculturated counterparts (USDHHS, 2009). Detrimental patterns of increased substance abuse among acculturating Latino immigrants appears to have a stronger relative effect on women than on men (Gfroerer \& Tan, 2003; Marín \& Posner, 1995; Vega et al., 1998). It is hypothesized that because Latino men have a higher prevalence of alcohol use to begin with, the effects of acculturation lead to the closing of the gap between genders in substance use (Lara, Gamboa, Kahramanian, Morales, \& Hayes Bautista, 2005).

\section{Acculturative Stress}

As previously noted, one of the prominent factors attributed to declining health patterns among Latino immigrants has been acculturative process (Finch \& Vega, 2003; Vega \& Amaro, 1994). Specifically, acculturation, and in particular, acculturative stress, has been associated with rises in substance use and HIV-risk behavior patterns among Latino immigrants (Lara et al., 2005; Loue, Cooper, \& Fiedler, 2003). Acculturation has been defined as the manner by which immigrant's attitudes and behaviors merge with 
those of the predominant cultural group as a result of exposure to the new culture (Berry, 1997). A common experience among recent immigrants is feelings to stress resulting from the acculturation process (Finch \& Vega, 2003). This form of stress consists of psychological or social stressors experienced by individual due to an incongruence of beliefs, values, and other cultural norms between their country of origin and country of reception (Cabassa, 2003). Acculturative stress is usually brought about by factors such as legal status, language barriers, difficulties assimilating to beliefs, values, norms of the dominant culture, and perceived feelings of inferiority, and discrimination (Berry, 1997).

\section{Religious Coping among Latinos}

The literature identifies various coping strategies that individuals use in dealing with stressful situations. Coping "refers to the various ways in which people respond when confronting stressful situations" (Kazdin, 2000, p. 300). These processes can be either cognitive or behavioral in nature (Moos \& Schaefer, 1982). The use of religion as a coping mechanism is a prevalent strategy among individuals, particularly among specific ethnic and racial subgroups (Chatters, 2000; Tix \& Frazier, 1998).

Religion and its features, such as prayer and church participation, appear to be very salient among most Americans. Approximately $95 \%$ of adults in the United States express a belief in God and about 88\% report the use of prayer (Hodge, 1996). As such, religion is used as a resource, particularly during times of adversity and emotional distress (Chatters, 2000; Finch \& Vega, 2003; Pargament, Koening, \& Tarakeshwar, 2001; Tix \& Frazier, 1998). This is particularly applicable in the Latino culture where religious values are known to be a pervasive force within the Latino culture, guiding attitude, behavior, and even social interactions (Abraido-Lanza, Vasquez \& Echeverria, 
2004; Fitchett, Murphy, Kravitz, Everson-Rose, Krause, \& Powell, 2007; Magana \& Clark, 1995; Plante, Maunel, Menendez, \& Marcotte, 1995). Not only is religion an important aspect of Latino culture, but when controlling for education and socioeconomic status, Latinos have been found to use religious coping mechanisms more frequently than their Non-Latino White counterparts (Valle, 1994). Furthermore, the use of religious coping is more prominent among less acculturated Latinos (Mausbach, Coon, Cardenas, \& Thompson, 2003).

Investigations into the influence of religion on HIV/AIDS have found religious factors to be inversely related to risky sexual behaviors (Ellison \& Levin, 1998). Similarly, considering oneself to be "not very religious" has been linked to greater probabilities of having multiple sexual partners among both men and women.

Additionally, men have shown a greater likelihood of being non-monogamous and engaging in HIV-risk behaviors (Elifson, Kline, \& Sterk, 2003).

In regard to substance abuse, the literature suggests an inverse relationship between religious resources and substance abuse (Chatters, 2000; Harrison, Koenig, Hays, Eme-Akwari, \& Pargament, 2001; Hodge, Anderek, \& Montoya, 2007). Researchers propose that religious involvement promotes mental and physical well-being by regulating behaviors in ways that decrease an individual's likelihood of engaging in health-risk behaviors. Mechanisms whereby religion impacts health is through (1) discouraging certain behaviors that increase the risk of health problems and (2) encouraging positive, low-stress lifestyles choices (Chatters, 2000).

Numerous studies have documented an inverse relationship between aspects of religious involvement and the use of substances including alcohol, tobacco, and illicit 
drugs (Hodge et al., 2007; Elifson et al., 2003; Koenig, 1998). However, the bulk of research in religious coping among minorities has been conducted among African Americans, women, and the aging populations (Prado, Feaster, Schwartz, Pratt, Smith, \& Szapocznik, 2004; Lewis-Coles \& Constantine, 2006).

In light of previous findings it stands to reason that the heavy use of religious coping by less acculturated Latinos could serve as a protective factor against acculturative stress and involvement in health risk behaviors. However, a dearth still exists as to how religion, and religious coping specifically affects behaviors among Latino immigrants. It is particularly essential to examine its effects on recent Latino immigrants that, as to our knowledge, have not been included in this area of study. Gaining insight into the role of religious coping in the lives of recent Latino immigrants may provide a crucial link towards our understanding of "the immigrant paradox."

\section{The Present Study}

This research addresses a fundamental gap in the existing literature regarding the influence of religious coping on the substance use and HIV-risk behaviors of Latino immigrants in the United States. The primary aims of this study were to examine (a) the influence of acculturative stress on substance use and HIV-risk behaviors among recent Latino immigrants, (b) whether religious coping mechanisms have a moderating effect on that relationship. The present study maintained a focus on how distinctive components of the religious coping process (i.e., internal, external, positive, and negative coping mechanisms) influence acculturative stress and its association with health risk behaviors.

This investigation utilized a cross sectional panel design. The data source for the current study consisted of secondary data from an ongoing NIH-funded longitudinal 
investigation of the influence of pre-immigration factors on the HIV and substance use trajectories of Latino immigrants. The use of a longitudinal data set including pre and post immigration data provided a unique opportunity to examine the relationship between the aforementioned variables.

\section{Theoretical Model}

The relationship between stress and health outcomes has been subject to extensive theoretical and empirical work. The stress process model is one of these frameworks, providing a strong foundation with over 20 years of research supporting its efficacy (Pearlin, Lieberman, Menaghan, \& Mullan, 1981; Lazarus \& Folkman, 1984). The stress process model has become a key theoretical framework for conceptualizing health disparities and risk and protective factors among minority groups. A fundamental underpinning of this framework is that the structural context of people's lives affects the conditions to which they are subjected. The basic premise of the model is that health outcomes related to stress are contingent not only on the extent of stress exposure, but also involve social and personal resources that serve as moderators and mediators influences in the link between stress and health outcomes (Pearlin, 1989; Turner, Lloyd \& Taylor, 2005). In essence, differences in current social conditions, which tend to be defined by social demographics such as gender, socioeconomic status, and ethnicity, affect exposure to stress, availability of coping resources, and in turn, stress outcomes (Pearlin, 1989).

\section{Research Questions}

The purpose of this investigation was to examine (a) the impact of acculturative stress on the substance use and HIV risk behaviors of Latino immigrants, and (b) whether 
religious coping mechanisms moderate the hypothesized relationship between the aforementioned variables. The following research aims and hypotheses were tested:

Aim 1: Determine the relationship of acculturative stress to substance use and HIV risk behaviors of recent Latino immigrants.

Hypothesis 1a: Latino immigrants with higher acculturative stress will have higher rates of alcohol use and HIV risk behaviors than recent Latino immigrants with lower acculturative stress.

Hypothesis 1b: Latino immigrants with higher acculturative stress will have higher rates of drug use than recent Latino immigrants with lower acculturative stress.

Aim 2: Determine the relationship of specific religious coping mechanisms to the acculturative stress experienced by recent Latino immigrants.

Hypothesis 2a: Latino immigrants who use internal, external, and positive religious coping at higher rates will experience lower acculturative stress. Hypothesis 2b: Latino immigrants who use negative religious coping at higher rates will experience higher acculturative stress.

Aim 3: Identify whether religious coping mechanisms moderate the relationship between acculturative stress and alcohol use/HIV risk behaviors among recent Latino immigrants. Hypothesis $3 a$ : The relationship between acculturative stress and alcohol use/HIV-risk behavior will be weaker among Latinos who report using more, external and positive religious coping. 
Hypothesis $3 b$ : The relationship between acculturative stress and alcohol use/HIV-risk behavior will be stronger among Latinos who report using more negative religious coping.

Hypothesis 3c: The relationship between acculturative stress and drug use will be weaker among Latinos who report using more, external and positive religious coping.

Hypothesis 3d: The relationship between acculturative stress and drug use will be stronger among Latinos who report using more negative religious coping.

This investigation is expected to make a contribution towards the understanding and elimination of health disparities within the Latino community. The present study can inform research that can lead to the development of new substance use and HIV prevention programs incorporating Latino values into culturally grounded interventions. Examining the role of religious coping in the acculturation process, and in the Latino culture as a whole, can serve as an important addition to the field of social work research, practice and policy. 


\section{CHAPTER II. REVIEW OF RELEVANT CONSTRUCTS}

\section{Religion in the Latino Culture}

Religion has played an influential role in the cultural and historical development of Latin America. Dating back to the Spanish conquest of Mexico and Central and South America, religion has shaped the worldviews of people living in Latin American for over 500 years. As such, religion has become an integral part of Latin American culture

providing both spiritual and practical aid (Skinner, Correa, Skinner, Baily, 2001). Studies have revealed that religion is used for assistance, support, socialization and has historically provided hope and meaning to Latinos during difficult life circumstances (Espinosa, Virgilio, \& Miranda, 2003; Skinner et al., 2001).

Religion continues to play an influential role in the lives of U.S.-born and immigrant Latinos. In the U.S., religious places of worship are central institutions within Latino communities. In an account of the role of churches in U.S. Latino communities Crane (2003) states:

The Latino church has played a crucial role of mediating between marginalized groups and the institutions of dominant society...Latino church[es] are important resource institutions that provide not only spiritual but also practical needs, and can function as communities of resistance to larger threatening forces. (p.3) The Latino church not only serves as a place of worship but also provides tangible resources through church programs that assist community members in obtaining jobs, shelter, furniture, schooling, and aid with immigration papers (Crane, 2003). These religious institutions can be particularly beneficial to Latino immigrants as a means of 
maintaining their identity and cultural roots as well as coping the multiple life changes and stressors of the immigration process (Gonzalez-Morkos, 2005).

Furthermore, Hernandez (1999) describes the spiritual significance of the churches within Latino communities in the U.S. in the following manner:

The Latino church stands at the very center of the community serving, enacting rituals of hope and meaning, transmitting values, enabling leadership, organization...motivating to transform personal and communal life, and inspiriting and idealism for a better future. (p. 18)

Among Latinos in the U.S., religious traditions are connected with family, friends, and even folk healers (Skinner et al., 2001). Religious ceremonies are considered social events, which strengthen bonds among family and friends as well as extended support systems (Gonzalez-Morkos, 2005). During stressful times, Latino families often rely on religious practices and beliefs to assist in the coping process (Guarnaccia, Parra, Deschamps, Milstein, \& Argiles, 1992). Latin American religious practices dating back hundreds of years, such as "peregrinaciones" (pilgrimage), "novenas" (nine-day festivities to venerate a saint), "mandas" (promises made to a saint as exchange something), and religious counseling conducted by a religious leaders are all parts of cultural norms that are practiced by Latinos in the U.S. as a means of dealing with life struggles (Skinner et al., 2001).

Although many Latino families are affiliated with a certain religious institutions such as the Catholic Church, some also consult natural healers when faced with mental health, medical problems and crisis (Vega, 1980). An example of this is the use of folk traditions such as Curanderismo and/or Santeria as a means of obtaining spiritual insight 
(Lucas, 1981). These beliefs are prevalent among Latinos and considered an important part of Latino culture and worldview. These traditions are widely recognized as indigenous mental health support systems in the Latino community (Baez \& Hernandez, 2001). It is therefore likely that Latino immigrants may continue to rely on these religious beliefs or folk traditions to obtain support and to interpret aspects of their changing lives in the United States (Skinner et al., 2001).

In 2006 the Pew Hispanic Center and the Pew Forum on Religion \& Public Life collaborated on a series of public opinion surveys regarding religion among Latinos in the United States (Pew Research Center, 2007). The Pew Forum U.S. Religious Landscape Survey consisted of over 4,600 interviews, and was one of the largest data collection efforts conducted on this subject. The surveys undertaken for this study, as well as previous research all point to the same basic distribution of adult Latinos by religious denomination. Consistently, findings indicate that about two-thirds of Latinos are Catholic, approximately a fifth practice some form of Protestantism and slightly less than a tenth identify as secular (Pew Research Center, 2007). Latinos not only constitute the largest minority group in the U.S. but also the largest group of U.S. Catholics with an estimated one-third (33\%) of all Catholics in the United States now represented by Latinos. Specifically, $67.6 \%$ of Latinos identity as Catholic, in comparison to $22.4 \%$ of Non-Hispanic Whites and 4.2\% of Non-Latino Blacks. Conversely, only $19.6 \%$ of Latinos identify as Protestants in comparison to $57.1 \%$ of Non-Latino Whites, and $82.9 \%$ of Non-Latino Blacks (Pew Research Center, 2007).

According to the Pew Forum U.S. Religious Landscape Survey, about two-thirds $(68 \%)$ of Latino Catholics are foreign born, while approximately half (55\%) of Latino 
evangelicals are immigrants (Pew Research Center, 2007). Latino immigration has served to considerably add to the number of Catholics in the US. Furthermore, foreignborn Latinos are most likely to attend Latino-oriented churches and comprise the largest share of Latinos who worship at such churches. Foreign born Latinos are by no means, however, the only church-goers that attend these predominantly Latino churches. In fact, two-thirds of Latino worshipers attend churches with Latino clergy, services in Spanish and heavily Latino congregations (Pew Research Center, 2007). A large number of USborn Latinos, as well as those that speak little to no Spanish, also report attending churches with Latino ethnic characteristics. While individuals who live in Latino communities are most likely to report attending Latino-oriented churches, there is a smaller but substantial share of Latinos who live in areas where the Latino presence is sparse, that also report attending these ethnic churches. Thus, Latino-oriented churches are neither exclusively a product of immigration or residential settlement patterns. Nevertheless, foreign-born Latinos are most likely to attend Latino-oriented churches and to comprise the largest amount of Latinos who worship at such churches (Pew Research Center, 2007).

\section{The Religion-Health Connection}

The past two decades of social work literature has documented the protective influence that religious involvement on the health behaviors of individuals (Chatters, 2000; Ellison \& Levin, 1998; Koenig, 1998). Nevertheless, when compared to other social and psychological constructs, inquiries into the effects religion has on lives of individuals is still in its formative stages (Hill \& Pargament, 2008). This dearth in research is especially pronounced among Latino populations. Most investigations into 
association between religion and health among Latinos have been epidemiological in nature and have focused almost exclusively on Mexicans living in the South Western United States (Arnada, 2008; Hill, Angel, Ellison, \& Angel, 2005; Levin \& Markides, 1985). This research suggests that religious attendance among Latinos is positively related to higher life satisfaction (Levin, Markides, \& Ray, 1996; Markides, Levin, \& Ray, 1987) and negatively associated with mortality (Hill et al., 2005). However, the effects of religious resources on health behaviors related to substance use and other HIVrisk among Latinos has been documented to a much lesser degree (Amaro, MagnoGatmaytan, Melendez, Cortes, Arevalo, \& Margolin, 2010). This is particularly true with recent Latino immigrant samples where associations between substance use/HIV risk and religion are relatively unknown.

\section{Religion and HIV-Risk Behaviors}

Behavioral risk factors for HIV infection include intravenous drug use, multiple sexual partners, sex with an intravenous drug user, sex in exchange for money or drugs, sex with an HIV positive partner, male-to-male sex, and, for women, sex with a partner who had sex with other males (Anderson, Mosher, \& Chandra, 2006; Whiteside, 2008). Religious involvement has been widely studied as a cultural/social factor that influences various health behaviors (Chatters, 2000; Gillum \& Holt, 2010). There is substantial literature documenting an inverse relationship between religiosity and substance use. Yet, little is known about the effects of religious factors on HIV-risk behaviors (Gillum \& Holt, 2010). Moreover, existing studies in the area of HIV/AIDS and religiosity have primarily focused on African American HIV positive samples (Prado, Szapocznik, 
Maldonado-Molina, Schwartz, \& Pantin, 2008; Scarinci, Griffin, Grogoriu, \& Fitzpatrick, 2009).

Research has shown greater religiosity to be associated with less involvement in high-risk sexual behaviors and less-permissive sexual attitudes (Edwards, Fehring, Jarrett, \& Haglund, 2008; Elifson et al., 2003). Similarly, considering oneself to be "not very religious" (compared to being "very religious") has been linked to greater probabilities of having multiple sexual partners among both men and women (Edwards et al., 2008; Elifson et al., 2003).

A number of studies in the U.S. have examined the association between religious involvement, such as church attendance, and risky sexual behavior on college samples (Burdette, Ellison, Hill, \& Glenn, 2009; Lefkowitz, Gillen, Shearer, \& Boone, 2004; Simons, Burt, \& Peterson, 2009). Findings from these investigations suggest a negative relationship between religious factors and HIV risk behaviors. Conversely, with U.S. Latino college samples religious involvement has not shown to be a significant predictor of unprotected sex (Fierros-Gonzalez \& Brown, 2002; Jemmott, Jemmott, \& Villaruel, 2002).

In regard to non-college samples, research with both women and men have yielded similar results, with religious involvement being inversely related to HIV risk behaviors (Sterk, Klein, \& Elifson, 2004). For instance, in a predominantly African American sample Sterk and colleagues found women who were less religious were more likely to engage in sexual HIV risk behaviors than those that were more religious (Sterk, Klein, \& Elifson, 2004). Nevertheless, two other investigations on non-college adult samples of White, Latina, and African American women from varied socio economic 
backgrounds found religious involvement to have no association with contraceptive decision-making (Wyatt, Carmona, Loeb, Guthrie, Chin, \& Gordon, 2000) or risky sexual behavior (Wayment et al., 2003).

In a more recent study, Gillum \& Holt (2010) used national survey data to examine the influence of religious involvement on HIV-risk behaviors among people in the U.S. The sample consisted on almost 10,000 males and females ages 15-44 from various racial/ethnic and socio demographic backgrounds. Women who never attended religious services had over two times greater odds of reporting HIV risk behaviors than for those who attended services weekly after controlling for age, race/ethnicity. No significant associations were found in men.

Studies of religiousness and HIV risk factors related to illegal drug use have also yielded inconsistent findings. In a predominantly middle-aged sample of male and female White, Latino and African American heroin and cocaine users, Avant and colleagues (2003) found that strength of religious faith and belief in a benevolent and meaningful world were both predictors of safe sex behaviors, yet had no association with drug-related HIV prevention behaviors (Avant, Marcotte, Arnold, \& Margolin, 2003). Furthermore, other studies have yielded results suggesting religious factors to be predictive of increased drug related HIV risk behaviors. Hasnain and colleagues (2005) investigated associations between religiosity and HIV risk behaviors in a sample of drug using African American men and women in a low income Chicago community. Results indicated that higher levels of religiosity were associated with higher rates of sharing injection materials (Hasnain, Sinacore, Mensah, \& Levy, 2005). 
In summary, although some results have been inconsistent, most investigations into the influence of religion on HIV have found religious factors to be inversely related to risky sexual behaviors (Ellison \& Levin, 1998). Further studies, particularly, with Latino samples are necessary in order to gain better insight into the role that religiosity plays in the engagement of HIV-risk behaviors.

\section{Religion and Substance Use}

Unlike the scarcity in research on HIV risk and religion, over the past 20 years there has been a rise in scholarly attention devoted to the role of religiosity on substance use. The literature suggests an inverse relationship between religious resources and substance use (Chatters, 2000; Harrison et al., 2001; Hodge, Andereck, \& Montoya, 2007). Numerous studies, including national survey data, have documented a link between religious involvement and lower rates of alcohol and illicit drug use across race/ethnicity and gender (Elifson et al., 2003; Hill, Burdette, Ellison, \& Musick, 2006; Hodge et al., 2007; Kendler, Liu, Gardner, McCullough, Larson, \& Prescott, 2003; Michalak, Trocki, \& Bond, 2007; Rote \& Starks, 2010).

Although current investigations have examined the associations between multiple dimensions of religiosity and substance use, studies focusing on the influence of religious coping on these behaviors are still needed (Bazargan, Sherkat, \& Bazargan, 2004;

Desrosiers \& Miller, 2008; Menagi, Harrell, \& June, 2008 ). Using the WORCS scale, Daugherty \& McLarty (2003) investigated the relationship between religious coping and types of drinking motivation in a sample 178 college students. Findings suggested an inverse association between the amount of alcohol consumed and religious coping scores. Robinson and colleagues (2007) examined change in alcoholic's religiousness from 
treatment entry to 6 months in a predominantly White sample of 123 outpatients with alcohol use disorders. Results indicated a statistically significant increase in religious coping along with decreases in alcohol use (Robinson, Cranford, Webb, \& Brower, 2007). Conversely, in a random sample of inner-city adult Latino and African-American emergency care patients Bazargan and colleagues (2004) found no association between religious coping and reports of alcohol consumption.

Due to the limited prospective data on religious coping and substance use, causal inferences between these variables are still in its formative stages. The existing research does suggest that religious involvement promotes mental and physical well-being by regulating behaviors in ways that decrease an individual's likelihood of engaging in substance use behaviors. Explanatory mechanisms by which religion is associated with positive health behaviors include the generation of positive emotions (i.e., love and forgiveness) and the provision of social and coping resources (Ellison \& Levin, 1998). Yet, investigations as to the influence of religious coping in the substance use patterns among Latinos remains particularly scarce.

\section{Religious Coping}

Religious resources not only serve as protective factors against substance use and other HIV risk behaviors, but also as a means of dealing with stressful life circumstances. Extensive evidence suggests that for many individuals religion is a way of deal with stress (Chatter, 2000; Ellison \& Levin, 1998; Pargament, 1997, 2002a, 2002b). These findings are indicative that some aspects of religion represent valuable resources for individuals facing difficult life circumstances. As such, religious coping styles have been found to be positively associated with better health status and may act as a protective 
factor against mental health problems and self-harming behaviors (Ano \& Vasconcelles, 2005; Chatters, 2000; Ellison \& Levin, 1998; Harrison et al., 2001).

The religious coping process involves the utilization of beliefs, practices, symbols, experiences, rituals, and action, in the coping process (Pargament, 1997). Pargament (2002a) defines religious coping as "[the] involvement of the sacred in the coping process...religious methods of coping are designed to address situations in which we are pushed beyond our immediate resources and confront our vulnerability to others, ourselves and our world" (p.241). In particular, people tend to look towards religion during highly stressful times. Empirical studies have shown that religiousness is generally intensified during these critical situations (Pargament, 1997).

According to Tix and Frazier (1998) religious coping is "the use of cognitive and behavioral techniques, in the face of stressful life events, that arise out of one's religion or spirituality" (p. 411). Examples of religious coping activities include prayer, confessing one's sins, and seeking strength or comfort from a higher being. It has been postulated that religious coping brings forth beneficial effects through various channels including: a) providing a framework of beliefs that facilitates cognitive restructuring of the meaning of an event, b) social support of a religious community, c) and a sense of control over stressful situations (Tix \& Frazier, 1998). Furthermore, Ellison and Levin (1998) find that individuals who turn to religious communities during stressful times gain tangible and intangible support mechanisms including emotional support and economic assistance. 


\section{Latinos and religious coping.}

Religious values are known to be a pervasive force within the Latino culture, guiding attitude, behavior, and even social interactions (Abraido-Lanza et al., 2004; Fitchett et al., 2007). Although the influence of other Latino cultural values such as respeto and familismo have been examined at length in the literature (Antshel, 2002; Kail \& Elberth, 2003), a dearth still exists as to how religion, and religious coping specifically affects behaviors such substance abuse and HIV risk behaviors among Latino immigrants. Moreover, the few investigations that have focused on the influence of religious coping on the health behaviors of Latinos have focused on primarily Mexican and Puerto Rican samples (Ellison, Finch, Ryan, \& Salinas, 2009; Finch \& Vega, 2003); leaving a striking scarcity in the literature regarding other Latino sub-ethnic groups.

Empirical investigations have demonstrated that religious coping is particularly used most among those groups that are disenfranchised in society including the elderly, less educated individuals, women, and other minority groups (Koenig, 1998; Pargament, 1997). A substantial amount of research has been conducted on the relationship between religion and the health risk behavior patterns of minorities. However, the bulk of these investigations have focused on the African American community (Hill \& Pargament, 2008; Lewis-Coles \& Constantine, 2006; Prado et al., 2004).

Religious coping styles may be particularly applicable for many in the Latino culture where religiosity is considered to be a central value (Magana \& Clark, 1995; Plante et al., 1995). Yet, few studies have examined the use of religious coping among Latinos. Furthermore, the limited literature that does exist offers contradictory conclusions regarding the influence of religious coping in the lives of Latinos. 
Dunn and O'Brien (2009) investigated the influence of religious coping on the psychological well-being of Central American immigrants residing in the Washington D.C. area. Results indicated no significant associations between the aforementioned variables. In a multi-site, longitudinal intervention program investigating the effectiveness of innovative interventions to support family caregivers, Latina caregivers residing in both Palo Alto California and Miami, Florida attended religious services more often, prayed more often, and used more religious coping than their non-Latino White counterparts (Coon et al., 2004; Mausbach et al., 2003; Rabinowirz, Hartlaub, Saenz, Thompson, \& Gallagher-Thompson, 2010). Abraido-Lanza and colleagues (2004) examined the use of religious coping among Latinos suffering from arthritis. The sample consisted of 200 Latinos that were predominantly foreign born and of Caribbean descent (Dominican, Puerto Rican, and Cuban.) Results indicated that religious coping was an important form of coping for this population. In a sample of Mexican immigrant farm workers, Hovey \& Magana (2000) found more frequent church attendance to be associated with lower rates of depression and suicidal ideation. More recently, Herrera and colleagues (2009) found negative religious coping to be associated with higher rates of depression among Mexican-American Latinos who cared for older relatives with longterm permanent disabilities (Herrera, Lee, Nanyonjo, Laufman \& Torres-Vigil, 2009).

Although many Latinos identify with a religion, and many enter the mental health system via the clergy (Ruiz, 2002), the ways in which religion functions within the coping process among Latinos remains unclear. In light of the documented extensive use of religious coping among Latinos, there appears to be a noteworthy gap of literature related to the use of religious coping among all Latino immigrant subgroups (Mexican 
Americans, Puerto Rican Cuban and South and Central American). Existing studies have focused primarily utilized Mexican (Finch \& Vega, 2003) and Dominican (AbaridoLanza et al., 2004) immigrant samples. As such, there is a scarcity of research in the role that religious coping plays in the lives of other Latino groups such as Caribbean, Central and South Americans (Alferi, Culver, Carver, Arena \& Antoni, 1999; Dunn \& O’Brien, 2009).

Existing investigations on religious coping among Latino immigrants have utilized immigrant samples that have been established in the United States for extended periods of time. The effects of religious coping in the lives of recent Latino immigrants in the United States has not been investigated . Furthermore, the influence that religious coping may have on the risk behaviors of this population has also yet to be explored.

\section{Conceptual models of religious coping.}

As the aforementioned studies suggest, religious coping has the potential capacity to significantly impact the physical and mental health of individuals. Theories as to how religious coping functions, however, are still in their nascent stages. The theories that have been postulated do not take race/ethnic difference into account, and are based on studies with predominantly non-Latino White samples. Kenneth Pargament is one of the leading contemporary religious coping theorists. Through the fusion of historical theoretical perspectives of religion, Pargament and colleagues (2000) identified five key functions of religious coping.

1. Meaning: Religion offers a framework of understanding an event in the face of difficult life experiences by facilitating cognitive restructuring of the meaning of that event. 
2. Control: Religion functions by providing a sense of control over a stressful episode. It provides a means of acquiring a sense of mastery and control to the individual who is facing stressful or difficult life circumstances.

3. Comfort/Spirituality: Religion allows for the connection with a force that is viewed as being greater than oneself. It functions by increasing comfort through a sense of closeness to a higher power, thereby creating a sense of defense and safety from distressing life events.

4. Social support: Religion plays a role in facilitating social cohesiveness, social solidarity, and social identity. It provides a sense of belonging, companionship, and intimacy with others, through spiritual connections and methods. These include, but are not limited to spiritual support from clergy or congregation members.

5. Life transformation: Religion assists individuals in making major life transformations. It facilitates the transition of releasing former objects of value and finding new sources of significance.

Moreover, Pargament and colleagues (1988) distinguished between three religious approaches to the coping process that influences an individual's sense of control over a stressful situation:

1. Self-directing approach: coping style in which people rely on themselves and their "God-given" resources in coping. This style has been linked to a greater sense of personal control and higher self esteem

2. Deferring approach: coping style in which people passively refer the responsibility of problem solving to a God/Higher Power. This style has been 
linked to a lower internal locus of control, lower self-esteem, and poor problem solving skills

3. Collaborative approach: coping style in which people work together with God/Higher Power in the problem-solving process. This style has been linked to a greater internal locus of control and greater self-esteem, and physical and mental health outcomes.

Several models have also been postulated in attempting to explain the welldocumented relationship between religious coping and health outcomes. Chatters (2000) identified five basic models representing possible relationship between stress and religion.

1. Stress response model: proposes that the presence of stress encourages and individual to mobilize or increase their frequency of religious behaviors (i.e., church attendance, prayer, etc.). This, in turn, function as a means of reducing stress and suppressing any physical or mental health effects that could have been brought upon by the stress.

2. Distress-deterrent model: suggests that stress and religion have independent and opposite salutary effects on health; the positive impact of religion on health occurs across levels of stress and partly offsets the harmful influence of stressors on physical and mental health.

3. Moderator model: represents the relationship between religion and stressors as contingent and interactive. This model does not propose any direct effects of religious involvement in health outcomes. Rather, it suggests that religion operates to moderate the effect of stress on health. According to the moderator 
model, people under exceptionally high levels of stress obtain the greatest health benefits from religious support and coping. Hence, religion may have significantly less impact on health consequences in individuals under low levels of stress.

4. Prevention model: suggests that religion has both direct and indirect effects on health. Indirectly, religious behaviors such a prayer and church attendance influence lifestyle choices, health behaviors, and lower exposure to stressful situations. Directly, religious involvement may serve a stress-reducing function through aspects such as religious devotion or prayer.

\section{Positive and negative religious coping.}

As previously mentioned, religious coping is defined as both a cognitive and behavioral coping strategy. The literature also identifies specific religious coping styles that have been found to be adaptive and maladaptive. For instance, in developing the Brief RCOPE, a measure of religious coping, Pargament and colleagues identified two distinct religious coping styles-positive and negative religious coping (Pargament, Smith, Koenig, \& Perez 1998). Positive religious coping strategies include: a) reframing a distressful even so as to view it as a potentially beneficial opportunity for growth and learning and b) finding a sense of meaning to a negative situation through a sense of spiritual connectedness with a Higher Power (Ano \& Vasconcelles, 2005; Hill \& Pargament, 2008).

In contrast, individuals who utilize negative coping, also referred to in the literature as spiritual struggles, tend to have a more hostile and pessimistic worldview. Negative coping strategies include: a) attributing distressful situation as a punishment 
from a Higher Power, and b) strained relationships with one's congregation and clergy, and 3) demonic appraisals of a distressful situation. These coping styles often result in feelings of shame, guilt, and anxiety (Pargament, 2002b).

After controlling for socio demographic variables, positive and negative religious coping have demonstrated divergent implications for physical and mental health. Positive religious coping has been associated with lower levels of depression and anxiety (Pargament et al., 2000) increased quality of life, higher levels of self-esteem (Ano \& Vasconcelles, 2005) and stress-related growth (Pargament et al., 1998). Conversely, negative religious coping has been associated with increased rates of anxiety (McConnell, Pargament, Ellison, \& Flannelly, 2006), depression, emotional distress (Fitchett et al., 2004; Herrera et al., 2009; Szymanski \& Obiri, Saenz, 2010), lower quality of life, decreased sociability (Chatters, 2000), lower self-esteem (Pargament et al., 1998), and physical health risk behaviors (Rabinowitz et al., 2010) as well as increased risk of mortality (Pargament et al., 2001).

Most investigations on positive and negative religious coping have been conducted with predominantly White samples (Abernathy, Chang, Seidlitz, Evinger \& Duberstein, 2002; McConnell et al., 2006). However, in a meta-analyses of religious coping and psychological adjustment to stress Ano and Vasconcelles (2005) found that positive religious coping was positively associated (and negative coping inversely associated) with better psychological adjustment in various minority populations including African Americans, Asians, Pacific Islanders and Latinos.

Specifically, investigations into the positive and negative religious coping styles among Latinos are scarce. Two recent investigations have examined associations 
between religious coping and physical/mental health outcomes among Latina (primarily Mexican) caregivers of elderly relative with and without dementia (Herrera et al., 2009; Rabinowitz et al, 2010). Herrera and colleagues (2009) found negative religious coping predicted greater depression among the Latina caregivers. Findings from an investigation by Rabinowtiz and colleagues (2010) suggest that negative religious coping styles are associated with greater physical health risk behaviors (i.e., poor diet, weight gain). Conversely, positive religious coping was related to better health behaviors. Latina caregivers also used positive religious coping more frequently than their non-Latina White counterparts.

\section{Internal and external religious coping.}

In addition to positive and negative religious coping, the literature distinguishes between two other forms of religious coping described as internal and external religious coping. Research suggests that individuals use specific religious cognitions (internal religious coping) and behaviors (external religious coping) as a means of dealing with stress (Ano \& Vasconcelles, 2005; Boudreaux, Catz, Ryan, Amaral-Melendez, \& Brantley, 1995; Chatters, 2000). Internal religious coping involves primarily private coping strategies such as engaging in contemplative prayer or counting one's blessings. Prayer and other internal religious coping have been found to alter how an individual assesses a potentially stressful situation (Ano \& Vasconcelles, 2005; Chatters, 2000). In essence, leading religious persons to re-evaluate stressful situation and view them as opportunities for spiritual growth, learning, or as part of a broader divine plan (Hill \& Pargament, 2008). 
External religious coping primarily social behavioral strategies such as seeking assistance from religious leaders or becoming involved with church activities. This style of coping facilitates an individual's ability to tap into external religious resources such as pastoral counseling and church programs (Chatters, 2000; Hill \& Pargament, 2008). External religious coping has been found to provide individuals with access to social support, social integration, tangible or instrumental aid (e.g., good and services) (Chatters, 2000), and socio-emotional assistance (Ellison \& Levin, 1998).

Investigations into the use of internal/external religious coping strategies have primarily been conducted with White (Boudreaux et al., 1995) and African American samples (Bishop, 2007). A dearth of literature exists on the internal and external religious coping strategies with Latinos (Alferi et al., 1999), and among Latino immigrants in particular (Ellison et al., 2009).

\section{Measures of religious coping.}

Despite the development of conceptual models to understand the role of religious coping on behaviors, there exists a lack of measurement to do so. Historically, empirical approaches to the measurement of religious coping have been limited and oversimplified. These measures have examined the construct from a more general perspective and have included: a) religious activities such as church attendance or prayer being used as proxy measures b) a single global measure such as asking participants if religion was "not at all involved" or "very involve" in their coping process with a stress situation, and c) employing one or more overtly religious item such as "I prayed" to more wide ranging scales of coping strategies (Harrison et al., 2001). 
Traditionally, these measures say little about the specific religious coping strategies that people utilize during stressful life situations. Researchers in the field have suggested that in order to better understand this construct it is important to examine the dynamic ways in which religion is utilized in the coping process (Ano \& Vascconcelles, 2005). According to Pargament, Koenig, and Perez (2000), "it is not enough to know that an individual prays, attends church, or watches religious television. Measures of religious coping should specify how the individual is making use of religion to understand and deal with life stressors" (p.521).

More recently, psychometricians have developed scales to measure religious coping mechanisms in a more comprehensive fashion. Five strategies have received the most attention: spiritual support/discontent, benevolent/punishing reframing, orientation to agency or control (self-directing, deferring, and collaborative coping styles), and the use of rituals (Fox et al., 1998; Harrison et al., 2001; Miner \& McKnight, 1999; Pargament, 1997).

Furthermore, scales examining the underlying patterns of religious coping strategies, typically divided into 'positive' and 'negative' religious coping styles, have recently been widely employed (Pargament et al., 1998). Measures of religious coping include: a) The Religious Problem-Solving Scale (Pargament et al., 1988), b) The RCOPE (Pargament et al., 2000), and c) The Brief RCOPE (Pargament et al., 1998). A particular weakness in most religious coping scales is that they have been predominantly validated on White samples, and therefore psychometric properties on diverse ethnic and racial are limited. A dissertation proposal completed by Gonzalez-Morokos (2005) obtained preliminary reliability analysis of a Spanish translation of the Brief RCOPE. Good 
psychometric properties were obtained. This study signified a step forward in the development of a culturally appropriate religious coping scale for Latinos. However, the study used a small sample of primarily Mexican immigrants. Validation of the measure with other Latino subgroups has yet to be completed.

\section{Acculturation}

Religious coping may be used by Latino immigrants as a means of dealing with the acculturation process. Acculturation occurs as a natural course of transition when an individual immigrates from their country of origin to a new country. With exposure to this new country comes an adaptation process known as acculturation. The classical definition of acculturation was presented by Redfield and colleagues (1936): "acculturation comprehends those phenomenon which results when groups of individuals having different cultures come into continuous first-hand contact with subsequent changes in the original culture patterns of either or both groups" (p. 149). According to Cabassa (2003) acculturation entails the social and psychological exchanges that take place between individuals from different cultures. These changes can be observed across a number of different domains such as attitudes, values, behaviors, and a sense of cultural identity (Cabassa, 2003). Berry (1997) conceptualizes acculturation in principle is a neutral term, where change may occur in either the dominant or minority group, or in both. In essence acculturation involves the exchange of cultural features that results when groups of individuals having different cultures come into direct contact with each other. This contact can lead to altered cultural patterns in either or both groups (Berry, 1997). 


\section{Conceptual models of acculturation.}

In order to understand the process of acculturation researchers have advanced several conceptual frameworks (Berry \& Sam, 1997; Cabassa, 2003). However, two predominant theoretical models that have dominated the study of acculturationunidimensional and bidimensional models of acculturation. Unidimensional models, sometimes referred to as a "zero-sum game" assume the acculturation process occurs along a linear continuum, ranging from not acculturated (complete immersion in the culture of origin) to totally acculturated (full immersion in the dominant culture) (Cabassa, 2003; Cortés, 1994; Cuéllar, Arnold, \& Maldonado, 1995; Marín \& Gamba, 1996). The unidimensional model assumes a loss of an individual's culture of origin as they merge into the dominant culture. This framework is based on the assumption that only the acculturating group is affected during this process. Essentially, unidimensional models view the acculturation process as a shift from one cultural domain towards another (Cabassa, 2003).

The unidimensional model has been criticized for what Cuellar et al. (1995) suggests is a false assumption that individuals must lose elements of their original culture during the acculturation process. In his critique of this model Cabassa (2003) states that "this zero-sum assumption leaves no room for the existence of two cultures within an individual and provides an incomplete and fragmented measure of this complex cultural process" (p. 133).

In contrast, bidimensional models view the maintenance of cultural values from country origin independently from the level of involvement that an individual sustains in the majority culture. In this model individuals may acquire specific values and customs 
from the majority culture, while simultaneously retaining those from the culture of origin. A distinctive characteristic of these models is the idea that an individual can be bicultural. Biculturalism occurs when an individual preserves their identity, beliefs, and values from their culture of origin, while learning, adopting, and integrating those from the majority culture. As such, this dual cultural identity preserves qualities from their culture of origin and simultaneously integrates values of the dominant society, while feeling equally comfortable in both (Lara et al., 2005).

Bidirectional models view the acculturation process as theoretically ranging from full participation to the full rejection of either of the cultural groups norms, values, attitudes, and behaviors. As conceptualized by Berry (1997) this model provides a framework that classifies acculturation strategies into four categories (assimilation, separation, integration, and marginalization. a) Assimilation-complete adherence to the values of the new culture, from the lack of desire to maintain those of the culture of origin; b) separation-maintenance of the culture of origin through rejection or avoidance to the new culture; c) integration-acceptance, adherence, and valuing of both cultures; and d) marginalization-a lack of opportunity or interest in engaging in the cultural values of either group. A parallel classification provided by Mendoza (1989) categorizes the acculturation strategies into four classes (cultural shift, cultural resistance, cultural incorporation, and cultural transmutation). a) Cultural shift—-the replacement of original cultural norms with those of the new culture b) cultural resistance-resisting the acquisition of new cultural norms, while maintain those of the culture of origin; c) cultural incorporation-integrating the customs from both the original and new culture; 
and d) cultural transmutation - shifting between the original and new cultural practices, thus creating a distinctive "sub-cultural entity" (Lara et al., 2005).

Marín (1992) views the acculturation process as a form of "culture learning" that can be divided into three distinct stages. a) The first level is superficial learning which consists of "the learning (and forgetting) of facts related to one's cultural history or tradition and changes in the consumption of food and in the uses of the media. These changes tend to be more superficial in nature in that they depend on the availability of these items versus the ones from the culture of origin. b) The intermediate level of cultural learning consists of changes in behavior, that are more fundamental to an individual's social life including language preference, ethnicity of social circles, neighborhoods, and preference for ethnic media. c) The last level of cultural learning is comprised of more intrinsically salient individual changes through adoption of values and norms of the majority culture. Nonetheless, these changes do not preclude the maintenance of norms from the original culture as well. For instance, in the case of familismo, a common value in the Latino culture, the needs of the family as a whole are viewed as superseding the needs of any particular family member. Certain aspects of familismo tend to change as individuals become more acculturated, such as a sense of obligation and the influence of family in directing the behaviors of its members. However, others such as the received and expected support from family, remain central to both highly and less acculturated Latinos (Sabogal, Marin, Otero-Sabogal, 1987).

Investigations into the acculturation process have predominantly utilized Mexican (Cavazos-Rehg, Zayas \& Spitznagel, 2007) and Puerto Rican Latino subgroups (Delgado, Lundgren, Deshpande, Lonsdale, \& Purington, 2008), and to a lesser degree Cubans 
(Wang, Schwartz, \& Zamboanga, 2010). However, studies examining the acculturation process of other Latino sub-ethnic groups are quite limited (Schwartz \& Zamboanga, 2008). This lack of research is notable in that the acculturation process can differ greatly across countries of origin. For instance, issues related to immigration status can greatly impact acculturation, and in particular acculturative stress. Documentation status can vary greatly across Latino sub-ethnic groups (Lueck \& Wilson, 2011). Whereas most Cuban immigrants are admitted under a special parole power that immediately grants them full legal status and puts them on a path to U.S. citizenship, other Latinos such as Mexicans, South and Central Americans may experience more hurdles in obtaining legal U.S. documentation. Research into the acculturation process across a wide range of Latino sub-ethnic groups are needed to better understand these differences.

\section{Measures of acculturation.}

To date, acculturation has been measured in a myriad of ways. Measures of acculturation have included a) participation in culturally specific behaviors such as diet and media usage, b) language preferences, c) knowledge of culture-specifics historical or current events, d) a sense of cultural identity, and e) the adherence to culture-specific values.

Unidimensional and bidimensional measures of acculturation have also been utilized and validated to differing degrees and published to date. These include Marin \& Gamba's (1996) Bidimensional Acculturation Scale for Hispanics, which taps into language use, linguistic proficiency, and electronic media usage. Cuellar and colleagues (1995) Acculturation Rating Scale for Mexican Americans-II (ARMSA-II) contains items on language usage, ethnic interactions, ethnic/national identification, and attitudes toward 
Latino and Anglo beliefs and behaviors. Additionally, Zea and colleagues (2003) created the Abbreviated Multidimensional Acculturation Scale, which assesses cultural identification, language competence, and cultural knowledge (Zea, Asner-Self, Birman, \& Buki, 2003).

Language preference has been one of the most commonly used proxy measures for acculturation in the research arena. However, the dependence on language items as a measure of such a complex process has its proponents and opponents. Supporters argue that psychometric analyses have demonstrated language, compared to other constructs, accounts for most of the variance in acculturation scales (Marín, 1992). In addition, although language is a multifaceted concept in itself, it is among the easiest and most straightforward acculturation constructs to measure (Marín, 1996). Vega and Gil (1998) describe language behavior as "very powerful for predicting sub-group level differences because embedded in the language is cultural imagery, values, knowledge of customs, and access to a cultural group and its respective artifacts. In short, language is a reference point for cultural allegiance and social expectations" (p. 128).

Critics have argued, however, that language does not fully encompass the multidimensional nature of the acculturation process, particularly among bicultural individuals. Marín \& Gamba (1996) state that among Latinos it is not uncommon to find people who are primarily English or Spanish speaking, regardless of their country of birth or length of residence in the U.S., as well as individuals who are fully bilingual. Furthermore, the case that an individual learns to speak English does not necessarily mean that they will cease utilization of the Spanish language, or vice versa (Marín \& Gamba, 1996; Lara et al., 2005). 
Apart from language other proxy measures for acculturation have included generational status (first, second, of third generation), age at time of immigration, foreign vs. U.S. born, and place of education. Yet, the chief assumption of these measures is that acculturation can be estimated by the amount of exposure an individual has to the majority culture (Cabassa, 2003; Lara et al., 2005; Negy \& Woods, 1992). Still another criticism has been the scarcity in adequate psychometric testing for new multifaceted acculturation scales as well as adaptations of previously validated measures (Marín \& Gamba, 1996).

Understanding and measuring acculturation can be a complicated and elaborate task. Ideally, this would involve moving past proxy measures and accounting for mediating and moderating influences within the acculturation process through validated multidimensional scales. Cabassa (2003) provides a framework of contextual factors that influence the acculturation process. He states that in order to "understand the acculturation experience of individuals one must inquire about the context in which the acculturation process takes place" (p.131). As shown in Table 1, Cabassa (2003) identifies contextual factors that should be taken into consideration include, preimmigration context (i.e., pre-immigration demographics and reason for immigration), immigration context (i.e., type of immigration group, route and duration of immigration), and settlement context (i.e., socio-political-economic environment, immigration policies of society of settlement, and legal status, age and demographics at time of settlement). A further modifier that should to be taken into account is the variability in the individual stress levels experienced by the immigrants during the acculturation process along with their coping capacities (Berry, 1997). 
While Redfield's classic definition of acculturation implies a bidirectional quality in acculturation, most measures to date have failed to capture the true nature of this construct, that according to Marín (1992) is "a rather fluid process that implies movement at different speeds across different dimensions (e.g., behaviors, attitudes, norms, and values, and planes)...that does not typically follow a deficit mode, but rather implies growth across a variety of continua."

\section{Acculturative Stress}

More recently, measures of acculturation have begun to incorporate a specific aspect of the construct known as acculturative stress. Under the discipline of crosscultural psychology, acculturation research experienced a shift in emphasis, from group to individual processes (Escobar \& Vega, 2000). In this light, acculturation has begun to be studied as a dynamic adaptation to stress and the development of coping mechanisms in dealing with the stress (Berry \& Sam, 1997). Researchers subsequently attributed this stress to negative experiences in the acculturation process. The term "culture shock" was coined to refer to this unique set of experiences. This concept later came to be known as acculturation stress. Berry, Kim, Minde, and Mok (1987) defined acculturative a stress "as a reduction in health status (including psychological, somatic and social aspects) of individuals who are undergoing acculturation, and for which there is evidence that these health phenomena are related systematically to acculturation phenomena" (p.491). It is a form of stress encountered by an individual due to an incongruence of beliefs, values, and other cultural norms between their country of origin and the country to which they have immigrated. Acculturative stress includes psychological and social stressors. Examples of social stressors include legal status stress and language barriers. Psychological 
stressors include difficulties assimilating to beliefs, values, norms of the dominant culture, as well as perceived feelings of inferiority, and discrimination (Cervantes, Padilla, \& Salgado de Snyder, 1991).

In a comprehensive concept analysis Caplan (2007) reviewed 19 articles related to acculturation stress within the past ten years. As seen in Table 2, this analysis yielded 3 dimensions of acculturative stress including: 1) instrumental and/or environmental stressors, 2) social and/or interpersonal stressors, and 3) societal stressors.

Caplan (2007) also identified contextual factors that moderate the influence of these dimensions. These include individual psychological characteristics and coping mechanisms, documentation status, socioeconomic status, and level of acculturation.

Individual characteristics and coping mechanisms: Adaptive functioning, selfesteem, and coping ability have been associated with whether acculturation stress is viewed as a negative versus a growing experience (Hovey \& Magana, 2000). In particular, the use of religion as a support mechanism has been associated with decreased stress for many immigrants (Finch \& Vega, 2003). It has also served a protective factor against depression and anxiety in first generation Mexican Americans (Hovey \& Magana, 2000).

Documentation status: Although most Latino immigrants experience instrumental stressors, undocumented immigrants appear to be experience acculturation stress to the greatest degree (Caplan, 2007). Perez and Fortuna (2005) used a sample of 230 Latinos utilizing a mental health facility in New York City to compare stressors between documented and undocumented immigrants. Findings indicated that undocumented immigrants experienced greater number of stressors including occupational and economic 
hardship and fear of deportation. Finch and Vega (2003) surveyed 3, 012 Mexican adults in a study of the links between social support, acculturation stress, and perceived stress. Results demonstrated stress related to documentation status had the most significant negative impact on perceived health, regardless of age, income, or level of acculturation.

Socioeconomic status: Lower levels of income have been cited as playing a prominent role in increased levels of acculturative stress among immigrants (Finch \& Vega, 2003).

Pre-immigration experience: The context in which the immigration occurred (voluntary versus involuntary) has also been linked to levels of acculturation stress. For instance, a forced move due to problems such as violence or political turmoil in the country of origin has been associated with greater levels of acculturation stress (Gil \& Vega, 1996). Salgado de Snyder and colleaugues (1990) conducted a comparison study of Central American versus Mexican immigrants. Central American immigrants who fled their home country due to civil war and human rights abuses were found to have greater psychosocial distress than their Mexican counterparts.

Level of acculturation: Lower levels of acculturation have consistently been associated with increased instrumental and/or environmental stressors (Caplan, 2007). Immigrants living with the United States for the least amount of years tend to experience the highest rates to daily stressors. Gil and Vega (1996) found that the relationship between time spent in the U.S. and acculturation stress is curvilinear. Levels of acculturation stress are highest with the first two years, decrease in years 3 to 10, and increase after this time point. 


\section{Conceptual model of acculturative stress.}

Berry and Kim (1988) provided a model of acculturation stress, which takes into account the context of acculturation, individual stressors, and individual perceived stress in regard to the acculturation process. These perceptions could be viewed as negative, benign, or as opportunities. Therefore, the stressors result from diverse experiences that are contingent on person's appraisal of the situation. Lastly, the model provides varying levels of acculturation stress, ranging from high to low, dependent upon the acculturation experience and stressors.

Berry and Kim (1988) model views the relationship between acculturation experience, stressors, and acculturation stress, not as deterministic, but rather moderated through other variables. These moderating factors include: a) the nature of the larger society (multicultural vs. assimilationist), b) type of acculturating group, c) modes of acculturation, d) demographic and social characteristics of the individual, and e) psychological characteristics of the individual (i.e., coping, attitudes).

\section{Measures of acculturative stress.}

Few measures of acculturative stress are currently available. The most commonly used scale of acculturative stress is the Hispanic Stress Inventory (HSI) (Cervantes, Padilla, A\& Salgado de Snyder, 1991). This measure was developed to asses a broad range of psychosocial stressors commonly experienced by U.S-born and immigrant Latinos. There is a 59 -item version for U.S. born individuals and a 73-item immigrant version for foreign-born individuals. The HSI includes four subscales:

Occupational/Economic Stress, Parental Stress, Martial Stress, and Family/Culture Stress. Additionally, the immigrant version includes the Immigration Stress subscale. 
Benet-Martinez (2003) also takes a multidimensional approach to measure this construct with her Riverside Acculturation Stress Inventory (RASI). This is a 15-item measure which gauges language skills, discrimination/prejudice, intercultural relations, cultural isolation and work challenges.

It has been recently suggested that current available measures of acculturation are flawed with factor analytic sub-scales confounding acculturative stress with acculturation constructs (Rudmin, 2009). It is anticipated that further research on acculturative stress as its own independent construct will lead to an increase in the design of measures for this variable.

\section{Acculturation and HIV Risk Behaviors among Latinos}

Immigration, while offering vast opportunities, is a stress filled process that can negatively impact the health behaviors of immigrants (Hilfinger, Deanne, \& Rubio, 2004). Previous research has linked acculturation to increased HIV risk behaviors among Latinos (Davila, 2000; Hines \& Caetano, 1998; Newcomb et al., 1998; Nyamathi et al., 1993). This literature indicates that certain Latino cultural beliefs may serve to facilitate or hinder self-protective behaviors that are critical to the prevention of HIV (VanOss Marin, 2003; Weidel, Provensio-Vazquez, Watson, Gonzalez-Guarda, 2008).

Research suggests that condom use tends to be lower among Latinos because of its perceived association with prostitution, sexually transmitted diseases, and extramarital affairs (Flaskerud \& Uman, 1993; Ford, King, Nerenberg, \& Rojo, 2001; Marin \& Marin, 1992). Moreover, due to gender role expectations, Latina women may be hesitant to negotiate condom use for fear of being perceived negatively by their sexual partners (Amaro, Vega, \& Valencia, 2001; Gomez, Hernandez, \& Faigeles, 1999; Romero, 
Arguelles, \& Rivero, 1993). These cultural beliefs, minimizing the importance of condom use and reinforcing gender inequality have been found to influence the HIV-risk behavior among Latino immigrants as well (Gomez, Hernandez, \& Faigeles, 1999).

The relationship between acculturation and HIV risk behavior is indeed a complex one. For instance, the literature reveals that among Mexican and Central American Latinos endorsement of native cultural and religious beliefs related to sex may decrease risk in one sense (e.g., limiting the average number of sexual partners) (Sabogal, Perez-Stable, \& Otero-Sabogal, 1995; Rojas-Guyler, Ellis, \& Sanders, 2005) but increase risk in another (e.g., decreasing the likelihood of being tested for HIV) (Kinsler, Lee, Sayles, Newman, Diamant, \& Cunningham, 2009). Current research with Latinos supports the complex relationship between acculturation and HIV risk behaviors. In one study, less acculturated Latina women had fewer sexual partners, but felt less empowered to discuss sexual issues with their partners (Amaro, 1993). Ford and Norris (1994) found lower levels of acculturation to be associated with decreased exposure HIV risk behaviors among youths of Mexican origin. Whereas various investigations suggest that less acculturated Latino men are more likely to endorse native condom beliefs and have extrarelational sexual partners (Billy, Tanfer, Grady, \& Klepinger, 1993; Sabogal, PerezStable, \& Otero-Sabogal, 1995).

Although investigations have begun to uncover the multifaceted relationship between acculturation and HIV risk, far less is known about how specific aspects of acculturation process affect these behaviors among Latinos. For instance, how acculturative stress affects the HIV risk behavior of Latino immigrants remains unclear. What is evident is that further insight into how acculturation impacts the subsequent 
health behavior of Latino immigrants is necessary given the elevated rates of HIV infection among Latinos in the U.S.

\section{Acculturation and Substance Use among Latinos}

There is well-established research demonstrating that exposure to social stressors, including acculturative stress, places individuals at risk for engaging in substance abuse behaviors. Vulnerable and disadvantaged populations have been found to experience exposure to these stressors at higher rates (Vega \& Amaro, 1994; Vega, Alderete, Kolody \& Aguilar Gaxiola, 1998; Turner \& Avison, 2003). Given that recent immigrants tend to be of relatively low socioeconomic status and experience higher levels of acculturative stress, it stands to reason that Latino immigrants should be at higher risk in relation to their native-born counterparts for engaging in such behaviors. Yet, research indicates that quite the opposite is true.

There is well-documented evidence indicating that recent Latino immigrants engage is substance use behaviors less than their more acculturated counterparts (Caplan, 2007; Lara et al., 2005; Turner et al., 2005). Furthermore, investigations into the substance abuse and acculturation patterns of Latinos shows vast discrepancies in the illicit drug use patterns between acculturated and non-acculturated Latinos. Recent national survey data reveal that Latinos with higher levels of acculturation are 13 times

more likely to report illicit drug use in comparison to their non-acculturated counterparts (USDHHS, 2009).

Researchers have hypothesized various explanations for this "immigrant paradox." One of the most prominent and well-established explanations relates to the acculturation process. It has been suggested that traditional Latino culture serves as a 
protective factor, and the loss or fading of these values through acculturation makes individuals more susceptible to engaging in high risk behaviors (Lara et al., 2005; Scribner, 1996). Moreover, acculturation often results in more contact with the majority culture, where experiences of discrimination, stressful social encounters (Vega \& Amaro,1994), and unmet aspirations (McKeever \& Clineberg, 1999) are more often the rule, than the exception (Finch \& Vega, 2003). Hence, low acculturation may serve as a buffer by limiting exposure to circumstances and situations that bring about social stress (Turner et al., 2005).

Although evidence exists that the immigrant paradox is relevant to various Latino subgroups, it is best documented within Mexican-Americans (Scribner, 1996). Convincing evidence for this phenomenon was first demonstrated by the Los Angeles site of the Epidemiological Catchment Area studies (Hough, Karno, Burnam, Escobar, \& Timbers, 1983; Robbins \& Regier, 1991). U.S. born Mexicans were found to have higher rates of several disorders including substance abuse and dependence, than their native born Mexican counterparts. Vega et al. (1998) confirmed the paradox in a study of U.S born vs. Foreign born persons of Mexican descent, where a prevalence of drug dependence among immigrants was found be less than a quarter of that observed for those born in the U.S.

Furthermore, research suggest that the drinking patterns of Mexican men in their native country is usually characterized by consuming larger quantities of alcohol, yet on fewer occasions than their U.S. counterparts. With acculturation, comes a fusion of drinking patterns that consists of continued consumption of large amounts of alcohol, except now at increased frequencies (Vega et al, 2003; Markides, Krause, Mendes de 
Leaon, 1988). This increase in alcohol consumption is followed by substance abuse related health disparities. As such, studies have reported mortality for alcohol related cirrhosis to be twice as high in Latino males in the U.S. in comparison to Non-Latino Whites (Galvan \& Caetano, 2003). Compared with non-Latino Whites, Latinos also have a higher prevalence of hepatitis $\mathrm{C}$, a serious infectious liver disease that greatly increases the risk for liver damage in heavy drinkers (Lieber, 2001; Stinson, Grant, \& Dufour, 2001). Disparities in substance abuse treatment utilization have also been cited between foreign-born and U.S. born Latinos, with immigrants being less likely to utilize treatment programs (Fisher, et al., 2004; Vega \& Sribney, 2005).

The negative effects of acculturation on drug use including marijuana, cocaine, and other illicit drugs, has been demonstrated in a range of Latino immigrant population including adult pregnant women, and adolescents (Amaro et al., 1990; Gfroerer \& De La Rosa, 1993; Gfroerer \& Tan, 2003; Vega et al., 1998; Vega \& Gil, 1998). Turner and colleagues (2005) investigated the association between nativity and drug dependence based on data from a representative sample of young adults of Cuban and other Latino ethnic groups in the South Florida area $(n=888)$. This study found Spanish language preference, which was used as a proxy for acculturation, to be the major factor in explaining $40 \%$ of the observed nativity difference in risk for drug dependence. As substantiated from previous research, this relationship was only found for women in the sample.

Investigation into the relationship between acculturation and substance use among various Latino subgroups have found varying effects across gender (Lara et al., 2005). In general, Latina females exhibit a general increase in alcohol and other drugs (AOD) use 
while Latino men increase in frequency of AOD use (Lara et al., 2005; Borges, et al., 2006; Gfroerer \& Tan, 2003). There is vast research corroborating findings of the stronger relative effects of acculturation on the rates of substance abuse among Latina females, in comparison to Latino males (Amaro et al., 1990; Gfroerer \& Tan, 2003; Lara et al., 2005; Marín \& Posner, 1995; Markides et al., 1998; Velez \& Ungemacjk, 1989; Zemore, Mulia, Ye, Borges, \& Greenfield, 2005). Reasons behind this discrepancy still remain unclear (Bethel \& Schenker, 2005). Nonetheless, hypotheses attempting to explain these gender-related differences have been put forward in. Among these postulations is that differing affects in exposure to acculturative stress and loss of cultural identity are associated with this phenomenon (Bethel \& Schenker, 2005).

Lara and colleagues (2005) provide a comprehensive review of the existing literature on the possible links between acculturation and health/behavioral outcomes among Latinos. Overall, one of the key findings of this review is that the impact of acculturation on Latino health and behaviors is very complex and not well understood. Although, certain positive and negative trends were found, effects were not consistently in the same direction, and many effects were mixed. Thus depending on the subject area, the measure of acculturation used, and socio demographic factors such as age, gender, and sub-ethnic group acculturation may have a negative, positive, or no effect on the health of Latinos. Furthermore, most of the studies reviewed were conducted among Mexican, Puerto Rican, and Cuban Latino sub-ethnic groups. The investigators recommended future research directed at examining how possible differences in the effects of acculturation experience across Latino subgroups relate to individual characteristics of among these respective subgroups. 
However, Lara et al. (2005) did find ample evidence suggesting that Latino immigrants living in the U.S., irrespective of country of origin, report higher rates of substance and alcohol use when compared to Latinos still living in the country of origin (Caetano and Mora, 1988; Cherpitel and Borges, 2001; Fosados et al., 2005; Vega et al., 1998). Moreover, the risk for substance use increases the longer they remain in the United States (Epstein, Botvin, Dusenbery, Diaz, \& Kerner, 1996). As previously mentioned, the negative effect of acculturation on substance abuse, although not completely uniform across areas, appears to have a stronger relative effect on women than on men (Amaro et al., 1990; Gfroerer \& Tan, 2003; Marín \& Posner, 1995; Markides et al., 1988; Vega et al., 1998; Velez \& Ungemacjk, 1989). It is hypothesized that because men have a higher prevalence of alcohol use to begin with, the effects of acculturation leads to the closing of the gap between genders in alcohol consumption (Lara et al., 2005).

Previous research has also demonstrated the effects that confounding variables can have on the relationship between acculturation and substance use. For instance, socioeconomic status (S.E.S.) often serves as a covariate in the link between acculturation and substance use. Using language of preference as a proxy for acculturation among a sample of Mexican Americans and Puerto Ricans, Amaro and colleagues (1990) found illicit drug use to be more highly correlated to acculturation among the individuals with a lower S.E.S., than in those with higher educational attainment. Velez \& Ungemack (1989) also found that lower S.E.S. was associated with higher rates of drug involvement by Latino adolescents in New York City. Conversely, findings from a recent study utilizing a large representative sample from a national 
database suggests higher education and income levels are associated with higher rates of alcohol use among Latinos (Alameida, Harrington, LaPlante, Kang, \& Taewoon, 2010).

Although ample evidence exists suggesting a positive relationship between substance use and acculturation, the literature to date lacks sufficient breadth and methodological rigor to make definitive evidence-based recommendations. First, measures that have been previously used to assess acculturation (i.e., language preference, generational status) are at best over simplified proxy variables used to evaluate a complex and multidimensional construct. Future research should attempt to incorporate measures of acculturation that encompass the complex bidirectional nature of acculturation. Secondly, incorporating the use of advanced statistical modeling techniques such as structural equations would further enhance our understanding of acculturation. The use of such models could lead to a better understanding of the effect of acculturation and differentiate between direct and indirect, or mediation effects. Thirdly, most research regarding acculturation has predominantly been done on Mexican, Puerto Rican, and to a lesser extent Cuban populations. Further research should include diverse Latino subgroups in an effort to shed light on the influence that country of origin may have on the acculturation experience.

\section{Acculturative Stress and Religious Coping}

To date most studies examining the effects of religious resources on the stress and health outcomes of minorities have focused on African Americans (Taylor, Chatters, \& Levin, 2004). Investigations with this population suggest that among African Americans, religious resources such as prayer and service attendance appear to temper the effects of discrimination, and other forms of psychological stress (Holt, Haire-Joshu, Lukwago, 
Lewellyn, Kreuter, 2005; Bowen-Reid \& Smalls, 2004; Wallace \& Bergman, 2002). To date, few studies have explored these relationships among U.S. Latinos (Ellison et al., 2009; Finch \& Vega, 2003). Thus, little is known about the relationship between religious coping and acculturative stress in this population. Furthermore, the investigations that have been completed have yielded conflicting findings.

Finch and Vega (2003) investigated Mexican-origin adults ( $\mathrm{n}=3012)$ and found that participants who engaged in religious support seeking behaviors, a component of external religious coping, experienced less acculturative stress, and were less likely to report being in poor health. In a more recent study using the same data set, Ellison and colleagues (2009) reported that religious involvement appeared to exacerbate the effects of acculturative stress on the depressive symptoms of these Latinos.

The Latino immigrant experience in the US is an inherently complex and diverse issue. However it is a topic that has been virtually ignored in the social work literature. In particular to the current research, the ways in which religion functions within the coping process among Latino immigrants remains unclear. The present investigation seeks to shed light on how religious coping processes are linked to acculturative stress and substance use/HIV risk behaviors of recent Latino immigrants.

\section{Summary of Current Study}

The primary aim of the present study was to examine the influence of acculturative stress on substance use and HIV risk behaviors among recent Latino immigrants, and to find whether religious coping mechanisms had a moderating effect on that relationship. A stress-process framework was applied to the conceptual model, research design, data analysis and interpretation. The investigation maintained a focus on 
how distinctive components of the religious coping process (i.e., internal, external, positive, and negative coping mechanisms) influences acculturative stress and its effects on risk behaviors. The study utilized a cross sectional panel design. Secondary data analysis from an ongoing longitudinal investigation of the influence of pre-immigration factors on the HIV and substance use trajectories of Latino immigrants was used in the present study.

\section{Conceptual Framework for Current Study}

The conceptual framework for the present study is the stress process model. Pearlin et al. (1981) formalized stress process model includes three essential concepts at the core of the stress process. These include a) stressors-which can be external, environmental or social factors, or internal, biological or psychological factors that create a need for adaptation or change. b) mediating/moderating resources- the social or personal resources that ameliorate the effects of stressors or modify the circumstances that are causing the stressors. The three types of moderators/mediators are coping strategies, personal resources and social support. Lastly, c) stress outcomes are the conditions resulting from stress exposure. These effects may take the form of biological, emotional, or psychological outcomes (Pearlin, 1989).

In describing the stress-process model in relation to substance abuse patterns, Turner et al. (2005) states "'to the extent that important differences in personal histories and in current social conditions tend to be conditioned by social statuses, including those of gender, ethnicity and nativity, the hypothesis follows that relationships between these statuses and substance abuse problems at least partially arise from status variations in exposure to stress" (p. 79). 
In determining the link between stress and health, the stress-process model takes into account the social context in which the stressor is experienced, a critical component of acculturation stress. Additionally, it incorporates the influence of social and personal resources, an integral element of the religious coping construct. Thus, the utilization of this model appears fitting in examining the proposed research questions.

\section{Research Aims and Hypotheses}

The purpose of the present study was to examine a) the impact of acculturation stress on the substance use and HIV risk behaviors of Latino immigrants, and b) whether religious coping mechanisms moderate the hypothesized relationship between the aforementioned variables. See Figure 1 for details on the research model. The following research aims and hypotheses are proposed:

Aim 1: Determine the relationship of acculturative stress to substance use and HIV risk behaviors of recent Latino immigrants.

Hypothesis 1a: Latino immigrants with higher acculturative stress will have higher rates of alcohol use and HIV-risk behaviors than recent Latino immigrants with lower acculturative stress.

Hypothesis 1b: Latino immigrants with higher acculturative stress will have higher rates of drug use than recent Latino immigrants with lower acculturative stress.

Aim 2: Determine the relationship of specific religious coping mechanisms to the acculturative stress experienced by recent Latino immigrants.

Hypothesis 2a: Latino immigrants who use internal, external, and positive religious coping at higher rates will experience lower acculturative stress. 
Hypothesis 2b: Latino immigrants who use negative religious coping at higher rates will experience higher acculturative stress.

Aim 3: Identify whether religious coping mechanisms moderate the relationship between acculturative stress and alcohol abuse/HIV risk behaviors among recent Latino immigrants.

Hypothesis $3 a$ : The relationship between acculturative stress and alcohol use/HIV-risk behavior will be weaker among Latinos who report using more, external and positive religious coping.

Hypothesis $3 b$ : The relationship between acculturative stress and alcohol use/HIV-risk behavior will be stronger among Latinos who report using more negative religious coping.

Hypothesis 3c: The relationship between acculturative stress and drug use will be weaker among Latinos who report using more, external and positive religious coping.

Hypothesis $3 \mathrm{~d}$ : The relationship between acculturative stress and drug use will be stronger among Latinos who report using more negative religious coping. 


\section{CHAPTER III. METHODS}

\section{Parent Study and Data Source}

The parent study that was the data source for the present research is the Recent Latino Immigrant Study (RLIS) (De La Rosa, Babino, Rosario, Valiente \& Aijaz, 2012). The Principal Investigator is Dr. Mario De La Rosa, director of the Center for Research on U.S. Latino's HIV/AIDS and Drug Abuse. This study was supported by Award number P20MD002288 from National Institute on Minority Health \& Health Disparities (NIMHD). The investigation is a longitudinal study that examines the HIV risk and substance use trajectories of recent Latino immigrants between the ages of 18 and 34 living in Miami-Dade County. The study began with a sample of $n=527$ baseline participants that were followed for a period of two years (three waves-baseline and two follow ups, 12 months apart). Data collection for the RLIS commenced in the Fall of 2008 and is expected to run through the Spring of 2012. Table 3 indicates the inclusion and exclusion criteria for the study sample. The rationale for the investigation included gaining better scientific understanding of the established rise in substance use and HIV risk behaviors among Latino immigrants as their time in the U.S. increases. A dearth of knowledge exists as to how pre-immigration factors influence the risk behaviors among this particular population. Investigating the role of pre-immigration factors on acculturative stress, substance use, and HIV risk behaviors is therefore the focus of the parent study. The aims of the RLIS were three fold:

Aim 1: Examine the influence of pre-immigration assets on post-immigration HIV risk behaviors among recent Latino immigrants over time. 
Aim 2: Identify the effects of pre-immigration assets on post-immigration acculturative stress, substance use, and HIV risk behaviors over time.

Aim 3: Investigate the relationship between pre-immigration assets, and post immigration acculturative stress, substance use, and HIV risk behaviors over time.

The parent study included standardized measures of acculturative stress, religious coping, social support, substance use and HIV risk behaviors, along with a variety of demographic variables, pre-immigration assets, social capital, and drug/alcohol/psychological treatment history. Those measures that did not already have a validated Spanish translated version were translated in- house. Specifically, the measures went through a process of a) translation/back translation, b) modified direct translation, c) and checks for semantic and conceptual equivalence to ensure accurate conversion from English to Spanish (Behling \& Law, 2000). In an effort to account for any within-group variability, the review panel conducting the modified direct translation consisted of individuals from various Latino subgroups representative of the Miami-Dade county population.

Data collection procedures consisted of a face-to face computer assisted personalized interviews. Interviews were completed in a location agreed upon by both the interviewer and participant. Most interviews were administered in participants' homes. Interviews were audio recorded for quality control purposes.

Data were cleaned, and entered into appropriate statistical software (Mplus/SPSS) databases for analysis. 


\section{Research Strategy}

The present investigation used a quantitative approach with a cross sectional research design utilizing secondary data drawn from the RLIS. Specifically, data was derived from wave 2 of data collection of RLIS. This wave of data was the first available wave that allowed for adequate examination of the proposed research questions.

Specifically, baseline data from the RLIS pertained to participant's behaviors before their immigration to the United States, wave 2 collected participant's behaviors after immigration. Approval for this study was obtained by Florida International University's Internal Review Board through an expedited review process.

\section{Procedures}

Participants in the RLIS were recruited through announcements posted at several community-based agencies providing legal services to refugees, asylum seekers, and other documented and undocumented immigrants in Miami-Dade County. Information about the study was also disseminated at Latino community health fairs and neighborhood activity locales (e.g., domino parks in the Little Havana section of Miami). Announcements were also posted around Latino communities and electronic bulletin boards such as Craig's List and an employment website that Latinos access to seek work in Miami-Dade County (see De La Rosa, Babino, Rosario, Valiente Martinez, \& Aijaz, 2012 for comprehensive description of recruitment efforts).

Respondent-driven sampling was the primary recruitment strategy for this investigation. This technique is an effective strategy in recruiting participants from hidden or difficult-to-reach populations (Salganik \& Heckthorn, 2003). For instance, undocumented Latino immigrants are often a hidden population due to the sensitivity of 
their legal status in the U.S. Given that approximately $25 \%$ of the U.S. Latino population consists of undocumented immigrants (Passel \& Cohn, 2008), and over 30\% of the baseline sample were undocumented immigrants, respondent-driven sampling was considered to be the most feasible sampling approach. This technique involved asking each participant (the seed) to refer three other individuals in their social network who met the eligibility criteria for the study and consented to be interviewed. Those participants were then asked to refer three other individuals. The procedure was followed for seven legs for each initial participant (seed), at which point a new seed would begin, thus limiting the number of participants that were socially interconnected. This process was undertaken in an effort to avoid skewing the respondent sample (Salganik \& Heckthorn, 2003).

Data were collected through computer assisted personal interviews. Participants were given the option to have the interview administered in English or Spanish. All interviews were conducted in Spanish. Interviews were completed in a location agreed upon by both the interviewer and participant. Most interviews were administered either in a participant's home (67\%), restaurant/coffee shop (14\%), or participant's work. The remaining interviews (19\%) were completed at the participants' work, school, or other public location. Interviews were audio recorded and reviewed by research assistants for quality control purposes.

\section{Participants}

Demographically, the sample consisted of men and women between the ages of 19 and 36 who currently reside in Miami-Dade County. This age group was selected for the Recent Latino Immigrant Study due to the documented elevated rates substance use 
and HIV risk among young adult Latinos in this age groups (Lara et al, 2005; Lipsky \& Caetano, 2009; Florida Department of Health, 2009). At the wave 2 interview all participants were Latino immigrants who have been living in the U.S. for less than two years. The participants' recent arrival to the U.S. is a unique and important component of this investigation. Although there are several studies in the literature that compare the substance use and HIV risk behavior patterns of immigrants who have been in the U.S. less than 5 years with those that have been in the U.S for more than 5 years (Brown et al., 2005; Blake et al., 2001; Gfroerer \& Tan, 2003), none of the studies exclusively began their data collection process on samples that had been in the U.S. for such a short period of time. The recent immigration status of the sample is an important element of the research design as acculturative stress has been found to be particularly high during the first two years of immigration (Gil \& Vega, 1996; Martinez, McClure, Eddy \& Wilson, 2011). Therefore, the recruitment of the sample in the early stages of immigration allowed for a better understanding of the effects of acculturation stress during a time when these stress levels are known to be at their peak.

Due to attrition, the current sample consists of 415 recent Latino immigrants, from the original baseline sample of $\mathrm{N}=527$. Documented reasons for attrition were as follows: a) 112 participants were unreachable with the contact information provided, b) 44 participants did not provide contact information c) 20 participants returned to their country of origin, d) 36 participants were deported, e) 10 participants refused to continue in the study, f) 1 participant was incarcerated, g) 1 participant went into the armed forces. The sample was $50.8 \%$ female and $49.2 \%$ male. All participants had immigrated to the United States approximately less than two years from the date in which the wave 2 
interview was conducted. The primary motive for immigration reported by participants was economic reasons (54.5\%), followed by reuniting with family members $(25.5 \%)$, political $(9.2 \%)$ and other $(10.6 \%)$. The ages of the participants ranged from 18 to 42 years $(M$ age $=28.68, S D=5.10)$. The education levels were as follows: $4 \%$ post graduate degree, $13 \%$ college degree, $35 \%$ some college, $38 \%$ high school or equivalent degree, and 10\% less than a high school. Participant's reported average annual income was $\$ 14,124.85(S D=\$ 12,411.50$, Mode $=\$ 6,000.00)$.

In terms of ethnic classification the most prominent ethnic group was Cubans at $50 \%$, followed by Colombians ( $18 \%$ ), Hondurans ( $8 \%$ ), Nicaraguans ( $7 \%$ ), and Venezuelans (3\%). Peruvians, Argentinians, Bolivians, Costa Ricans, Dominicans, Ecuadorians, El Salvadorians, Mexicans, Guatemalans, Uruguayans, and Panamanians each represented $2 \%$ or less of the sample. Approximately $80 \%$ of participants were documented immigrants, while $20 \%$ were undocumented.

\section{Sample Size Considerations}

The issue of sample size in SEM analysis is somewhat varied and controversial (Jackson, 2007). Various approaches to arriving at a sample size have been suggested, such as a minimum sample size (e.g., 200), having a certain number of observations per measured variable, or through conducting power analyses (e.g., MacCallum, Browne, \& Sugawara, 1996). Another suggested approach equates the necessary sample size to the number of parameters that must be estimated (e.g., Bentler \& Chou, 1987; Bollen, 1989; Kline, 1998, Marsh, Balla, \& McDonald, 1988; Tanaka, 1987; Ullman, 1996), where higher values of the ratio of observations to parameters to be estimated (N:q) are preferred (Jackson, 2003). 
To determine an appropriate sample size, structural equation modeling requires that in addition to statistical power, issues of the stability of the covariance matrix and the use of asymptotic theory be taken into account. In terms of power, it is difficult to evaluate the power associated with specific path coefficients in complex SEM models because of the large number of assumptions about population parameters that must be made (Jaccard \& Wan, 1996).

In terms of asymptotic theory and covariance stability, simulation studies tend to suggest that sample sizes of 100 to 125 or larger often yield adequate results given that reasonably reliable measures are used (reliabilities greater than 0.65 ) and with a reasonable number of indicators per latent variable (Jackson, 2003; Jaccard \& Wan, 1996; Kline, 2005). Furthermore, most sources will indicate that, depending on the model's complexity a researcher should have at least 200 cases (Martens, 2005). The sample size in the current study exceeds these standards.

\section{Measures}

Data collection and measurement tools were selected from the parent study for their relevance to the proposed research. Selected measures were standardized scales demonstrating adequate psychometric properties. Additionally, four of the six scales used in the present study have validated Spanish versions. Specifically, the Brief RCOPE (Gonzalez-Morkos, 2006), Timeline Followback (Sobell et al., 2001), AUDIT (Contel, Gual, \& Colom, 1999) and Hispanic Stress Inventory (Cervantes, Padilla, \& Salgado de Snyder, 1991) all had validated Spanish versions. The other two measures (Ways of Religious Coping Scale and Risk Behavior Survey) went through a thorough translation 
process as previously discussed including translation/back translation, modified direct translation, and checks for semantic and conceptual equivalence.

The following variables from wave 2 of the RLIS were examined: a) degree of acculturative stress in the past 12 months (Hispanic Stress Index), b) use of religious coping mechanisms (external, positive, negative) in the past 12 months (Brief RCOPE \& Ways of Religious Coping Scale), c) alcohol use in past 90 days (Timeline Followback) and past 12 months (AUDIT), d) other drug use in the past 90 days (Timeline Followback), e) sexual HIV risk behaviors in the past 90 days (Risk Behavior Survey). This 90-day recollection window was done to maintain data accuracy.

In addition to the aforementioned key variables, covariates that may influence the predictors and outcomes were explored. These covariates were chosen in accordance to the literature review in the previous chapter. Research suggests that the two primary sources on acculturative stress among Latino immigrants are legal status and socio economic issues (Finch \& Vega, 2003). Furthermore, there is abundant literature suggesting gender differences in alcohol and other drug use as well as sexual HIV risk behaviors among Latinos, with Latino men engaging in these behaviors at higher rates than Latina women (Hines \& Caetano, 1998; Lara et al., 2005). Given these previous findings income, education, legal status, and gender were explored as possible covariates in the present research model. Baseline estimates of the outcomes variables (preimmigration alcohol use, pre-immigration drug use, and pre-immigration sexual HIV risk behavior) were also included as covariates. 


\section{Sociodemographics}

A demographics form assessed, in part, participant time in the U.S., level of education $(1$ = less than high school, 2 = high school, 3 = some training / college after high school, 4 = bachelor's degree, 5 = graduate / professional studies), and income. For the present study an income variable was computed by dividing total household income in the past 12 months by the total number of people dependent on that income .

\section{Documentation status}

Legal status was measured by immigration category. Participants were asked to report their current legal status in the U.S. A total of ten possible categories were provided: a) permanent resident, b) temporary resident, c) without papers, d) temporary work visa, e) student visa, f) tourist visa, g) expired visa, h) dependent on someone else's visa (for example husband wife, parent or guardian, i) exile and j) temporary protected immigrant. These categories were then recoded into a dichotomous variable of legal (1) or undocumented (0) immigration status.

\section{Acculturative stress}

The validated, 18-item Spanish version of the immigration stress subscale of the Hispanic Stress Inventory Scale -Immigrant Version (Cervantes et al., 1990) was used to measure acculturative stress. This scale is a measure of psychosocial stress-event experiences for Latino immigrants. It has been widely used with this population (Ellison et al., 2009; Loury \& Kulbok, 2007). The participant reports whether or not they experienced a particular stressor $(0=$ no, $1=$ yes $)$. If the stressor was experienced, then a subsequent follow-up question is asked regarding the appraisal of how stressful that particular event was to the respondent assessed by a 5-point Likert scale format. $(1=$ not 
at all to $5=$ extremely). In cases where the stressor was not experienced the appraisal items was recoded to $(1=$ not at all $)$ (Cervantes et al., 1990). Example items are as follows: a) I felt guilty about leaving my family and friends in my home country; b) Because my poor English, it has been difficult for me to deal with day to day situations; c) Because I am Latino, I have had difficulty finding the type of work I want. Test-retest reliability coefficient for the Immigration Stress scale has been reported as $.80(p<$ .0001) with Cronbach's alpha coefficient equal to.85 (Cervantes et al., 1990). In the present study reliability estimates for immigration stress frequency and appraisal were $\alpha$ $=0.84$ and $\quad \alpha=0.87$ respectively. Given the very high correlation between frequency and appraisal of stress in the current sample $(r=.92)$, the sum of the immigration stress frequency and immigration stress appraisal scores was used to measure overall acculturative stress.

\section{Internal and external religious coping}

The Ways of Religious Coping Scale (WORCS) was used to measure internal and external religious coping. This instrument is a 25 -item questionnaire measuring religious coping cognitions and behaviors (Boudreaux et al., 1995). Items are divided into two subscales, external/social and internal/private religious coping. This instrument is in a 5-point Likert scale format. Responses range from $1=$ not used at all to $5=$ always. Example items from the external subscale include: a) I ask my religious leader for advice and b) I get help from church/mosque/temple members. Items from the internal subscale include: a) I find peace by sharing my problems with God and b) I pray for strength. Example items from the external subscale include: a) I ask my religious leader for advice and b) I get help from clergy. Internal consistency estimates reported in past studies of 
the WORCS are favorable: internal scale $(\alpha=0.95)$ and external scale $(\alpha=0.94)$

(Boudreaux et al., 1995.) The WORCS was initially validated with predominantly nonLatino, White, Christian college students (Boudreaux et al., 1995). Although the scale has been used with ethnic and racial minority groups, these samples have consisted largely of African American respondents (Bishop, 2007; Boudreaux et al., 1995). As a means of making the WORCS more culturally sensitive to our sample, some of the wording on a few items was expanded to broaden it from the Judeo-Christian perspective. For example "I recite a psalm" was expanded to read "I recite a psalm or other religious scripture" and "I talk to my minister/preacher/rabbi/priest" was expanded to "I talk to my minister/preacher/rabbi/priest/or other religious leader." The participants were also told that for items stating the term "God" what is being referred to is a higher being that is relevant to them. Spanish translation of this measure through aforementioned procedures was developed for the RLIS. In the present study the External/Social Religious Coping scale indicated high internal consistency with a Cronbach's alpha coefficient equal to 0.95 . The Internal/Private Religious Coping scale also indicated high internal consistency with a Cronbach's alpha coefficient equal to 0.96 .

\section{Positive and negative religious coping}

The Brief RCOPE is a widely used measure of religious coping and separates this construct into positive and negative religious coping styles (Pargament et al.,1998). Rather than measuring behavioral religious coping strategies, this instrument focuses primarily on cognitive religious mechanisms. It is a 14-item measure scored on a 4-point Likert scale with responses ranging from $1=$ not at all, $2=$ somewhat, $3=$ quite a bit, and $4=$ a great deal. Both the Positive and Negative Religious Coping subscales contain 7 
items each. Example items from the Positive scale include: a) Sought help from God in letting go of my anger and b) Looked for a stronger connection with God. Example items from the Negative Religious Coping subscale are as follows: a) Wondered what I did for God to punish me and b) Questioned God's love for me. Both subscales of the Brief RCOPE have shown to have high internal consistency: positive religious coping subscale ( $\alpha=0.90)$ and negative religious coping $(\alpha=0.81)$ (Pargament et al., 1998). A Spanish translated version which has been validated with Latino populations was used in the parent study (Gonzalez-Morkos, 2005). The Spanish version of the Brief RCOPE also contains favorable internal consistency: positive religious coping $(\alpha=.87)$, negative religious coping $(\alpha=.80)$. Correlational analysis between positive and negative religious coping scales were not significantly correlated $(r=.06, p=.734)$, indicating they measure distinct patterns of religious coping. The Spanish version of the Brief RCOPE validated by Gonzalez-Morkos (2005) utilized a Latino sample of predominantly Mexican descent. In the present study the Brief RCOPE also demonstrated good internal consistency: positive coping ( $\alpha=$.95), negative coping $(\alpha=.83)$.

\section{Alcohol use}

A three indicator latent variable was used to measure alcohol use (procedures for establishing latent construct are described in the Data Analytic Plan section below). An advantage to using latent variables is the ability to measure multiple dimensions of a behavior. Latent variables are also free of random error because it is estimated and removed (Bollen, 1989). The three indicators for the alcohol use latent variable consisted of the following: a) hazardous/harmful drinking patterns in the past 12 months, b) 
frequency of alcohol use in the past 90 days, c) quantity of alcohol use in the past 90 days.

Hazardous/harmful drinking patterns was measured through the Alcohol Use Identification Test (AUDIT; Babor, Biddle-Higgins, Saunders \& Monteiro, 2001). The AUDIT is a 10-item screening questionnaire with 3 questions on the amount and frequency of drinking, 3 questions on alcohol dependence, and 4 on problems caused by alcohol. The AUDIT demonstrates sensitivities and specificities comparable, and typically superior, to those of other self-report screening measures (Babor et al., 2001). Test-retest reliability and internal consistency are also quite favorable in past studies (Cherpitel, 1997, 1998; Steinbauer, Cantor, Holzer \& Volk, 1998). Several studies also show them as correlated with biochemical measures of drinking (Dolman \& Hawkes, 2005). Indices of internal consistency, including Cronbach's $\alpha$ and item-total correlations, are generally in the 0.80s (Allen, Litten, Fertig, \& Babor, 1997). Similar reliability estimates for the AUDIT were obtained in the present study $(\alpha=.81)$.

Frequency and quantity of alcohol use in the past 90 days was measured through the Timeline Followback Interview (TLFB) (Sobell \& Sobell, 1992). This measure is a widely-used and well known research and clinical assessment tool with acceptable reliability and validity for various groups of individuals with substance use problems. The TLFB was used to collect data of substance in the last 90 days prior to assessment. With the TLFB substance consumption information is collected using a calendar format to provide temporal cues (e.g., holidays, special occurrences) to assist in recall of days when substances were used. With regard to alcohol, daily use information was collected in-number of standard drinks per day. The TLFB has a standardized Spanish version and 
has been widely used with Latino populations (Gil et al., 2004; Dillon, Turner, Robbins, \& Szapocznik, 2005). The TLFB has been found to be a reliable and valid measure, demonstrating high correlations with biological markers and collateral reports with Latino populations (Dillon et al., 2005).

Frequency of alcohol use was calculated by summing the total number of days alcohol was consumed in the 90 day window. Quantity of alcohol was calculated by average number of drinks that were consumed on days that alcohol was drank in the past 90 days.

\section{Other drug use}

The TLFB was also used to collect data of frequency of other use in the last 90 days prior to assessment. Participants were given 9 categories of drugs to choose from: a) cannabis (marijuana, hashish), b) cocaine (speedball, crack), c) sedative, hypnotics, or tranquilizers (valium, xanax), d) stimulants (amphetamines, speed, [ecstasy, E, roll], crystal meth [tine, ice], e) heroin, f) opioids (morphine, methadone, codeine), g) hallucinogens (LSD, mesculine, mushrooms), h) inhalants (glue, paint, amyl nitrate) and i) anything else. Frequency of drug use information was collected in number of days drugs were used.

\section{Sexual HIV risk behaviors}

The Risk Behavior Assessment (NIDA, 1993) was used to measure sexual HIV risk behaviors. This scale contains sexual and drug related behaviors related to HIV risk. For the present study, items regarding frequency of condom use during sexual encounters in the last 90 days were used. Items for condom use during oral, anal, and vaginal sex 
were on a 4-point Likert scale consisting of $1=$ never, $2=$ less than half the time, $3=$ more than half the time, and $4=$ always. In order to ease interpretation of results the items were reverse scored so that a score of 0 indicated least HIV risk and 4 highest HIV risk. Psychometric properties for this measure are as follows: Sexual-practice questions, the coefficients ranged from $r=.66, \mathrm{SEM}=0.79$ for number of drug-injecting partners to $r=.83$, SEM $=3.29$ for number of days had sex in the last 30 days (Weatherby et al., 1994; Needle et al., 1995). The Risk Behavior Survey has been previously used with Latino samples (Delgado et al., 2008; Lundgren, Chassler, Ben-Ami, Schilling, \& Purington, 2005).

\section{Data Analytic Plan}

Statistical analyses were completed on SPSS and MPlus 6.0 software (Muthén \& Muthén, 2010). Descriptive statistics were performed for all variables to summarize data and profile all variables. Variables measured on non-metric scales (categorical variables) were summarized in terms of proportions while those measured in metric scales (numerical variables) will be summarized in terms of means and standard deviations. Examination of the distribution forms were pursued through graphical methods and summary statistics of higher order moments (skewness and kurtosis).

Frequency distributions for all continuous variables were conducted to determine if they violated the assumption of normality. According to Kline (2005), continuous variables were deemed non-normal if they yielded absolute skewness and kurtosis values greater than 3.0 and 8.0 respectively. Square root transformations were conducted with positively skewed variables (see Chapter 4: Results). Transforming data is a common 
practice in multivariate research to address outliers and non-normal distribution patterns (McDonald \& Ho, 2002).

The drug use variable had a non-normal distribution with only $7 \%$ sample reporting engaging in drug use at least once in the past 90 days. Thus, this outcome variable followed a Poisson distribution, where the most frequently occurring response is zero. As a result there is no way to transform this data to approximate a normal distribution. When $80 \%$ or $90 \%$ of participants report no engagement in the target behavior, zero-inflated Poisson (ZIP) models (Atkins \& Gallop, 2007) are recommended, in which the zeroes are modeled separately from the nonzero count data (Schwartz et al., 2011). A further explanation of this modeling technique is provided later in this chapter. Pearson and Spearman rho two-tailed correlation analyses were conducted to examine simple relationships among the variables in the model.

Statistical tests for were conducted to assess the potential influence of covariates in the research model. As previously mentioned, the covariates that were chosen in accordance to the literature were gender, education, income, and legal status. Preimmigration scores for the outcome variables (alcohol use, other drug use and HIV risk) were also controlled for in the present study in order to account for substance use and HIV risk behaviors that may have been present prior to immigration. An analysis of variance (ANOVA) was used to evaluate the potential influence of the categorical education variable. A Pearson's correlation analysis was used for the continuous income variable. Spearman's rho correlation analyses were conducted on the binary covariates (gender and documentation status). 
Confirmatory factor analyses (CFA) were conducted to test if hypothesized indicators adequately represented the latent constructs for alcohol use and sexual HIV risk. The procedures for CFA included model estimation, model fitting, and evaluation (guidelines are stated below). In addition, the CFA analysis included an assessment of path loadings, variance extracted, and construct reliability (Hancock \& Mueller, 2001; Kline, 2005).

The next step in the analysis was to test for measurement invariance across time for the pre and post immigration alcohol use latent constructs and the pre and post immigration HIV risk latent construct. Measurement invariance refers to the extent to which the same measurements conducted under different conditions (over time in this case) yield the same measures of the attributes under study (Bauer \& Curran, 2011). More formally, measurement invariance can be defined as having the expected values for the indicators of the latent constructs equal across groups. If measurement invariance is not present, it suggests that there are systemic differences in the ways that participants responded to the survey questions, such that the latent factors were not measured equivalently across both time points. For the present study it would mean that participants would score differently on pre-immigration alcohol use and HIV risk than at post immigration, even at equal levels of the latent constructs. In order to adequately control for pre-immigration alcohol use and HIV risk in the structural model, measurement invariance is necessary. If measurement invariance is not found, then the pre-immigration alcohol and HIV risk latent constructs cannot be used as covariates in the structural model due to the confounding influence of the significant measurement differences. 
In the present study the sequence for testing measurement invariance consisted of tests of configural and metric invariance (Horn \& McArdle, 1992; Meredith, 1993). Configural invariance establishes that the same number of factors and the same pattern of factor loadings characterize each group or time point (Bauer \& Curran, 2011).

Configural invariance requires that the same variables load on the same number and pattern of factors across groups or time (Horn \& McArdle, 1992). A measurement model testing for configural invariance would place no constraints, indicating factor pattern coefficients are free to vary. Configural invariance metric invariance establishes that the latent constructs have the same metric and origin (Bauer \& Curran, 2011). Metric invariance is tested by examining the equivalence of factor loadings across the preimmigration and post immigration estimates of the alcohol and HIV-risk values. This is done by imposing equality constraints across both time points. In the latter condition, meaningful quantitative group comparison can be made across time because similar measurement units are present across both waves of data (De Frias \& Dixon, 2005). Fit differences between the constrained and unconstrained models were evaluated using two standard indices: the difference in chi-square values $\left(\Delta \chi^{2)}\right.$, the difference in CFI $(\Delta \mathrm{CFI})$ values. In each model comparison, for the null hypothesis of invariance across groups to be statistically rejected, both of the following criteria had to be met: $\Delta \chi^{2}$ significant at $\mathrm{p}$ $<.05$ (Byrne, 2001), $\Delta$ CFI $>.01$ (Cheung \& Rensvold, 2002).

Given the distribution of the data two separate research models were tested in the present study. Both models were tested using MPlus 6.0 (Muthén \& Muthén, 2010) Structural equation modeling (SEM) was conducted to test the overall model fit for alcohol use and HIV risk outcomes (see Figure 5). Zero-inflated Poisson modeling, 
described below, was used to test the drug use outcome (see Figure 6). Overall model fit was evaluated following the recommendations of Bollen and Long (1993) using a variety of global fit indices, including indices of absolute fit, and fit indices with a penalty function for lack of parsimony. These include the traditional overall chi square test of model fit (which should be statistically non-significant. Note that for models with over 400 cases the chi square is almost always statistically significant), the Root Mean Square Error of Approximation (RMSEA; which should be less than 0.08 to declare satisfactory fit), the Comparative Fit Index (CFI; which should be greater than 0.95); and the standardized root mean square residual (which should be less than 0.05 ).

In addition to the global fit indices, more focused tests of fit will be pursued. These include examination of the standardized residual covariances (which should be between -2.00 and 2.00) and modification indices (which should be less than 10.00). The parameter estimates were examined inadmissible solutions known as Heywood cases, such as correlations greater than one or variances less than zero. Care was taken to ensure there is no specification error.

As previously mentioned, the drug use outcome followed a Poisson distribution, where the most frequently occurring response is zero. In order to take this distribution into account, zero-inflated Poisson (ZIP) modeling was used in testing research aims/hypotheses where drug use was the outcome variable (Hypothesis 1b, Hypothesis $3 c$ and Hypothesis 3d) (see Figure 6). In a ZIP model, the zero responses are modeled separately from the nonzero count data-which allows for detection of significant patterns that would otherwise go obscured by the preponderance of zero responses. ZIP models are set up by recoding the original variable into two new variables: a dichotomous 
(incidence) indicator reflecting whether or not the person engaged in the risk behavior: and a count (frequency) variable reflecting the number of times the person engaged in the behavior. For individuals who indicate no engagement in the behavior, the count (frequency) variable is set to the system-missing value (Schwartz et al., 2011). The drug use incidence variable indicated whether participants did or did not engage in any drug use behavior in the past 90 days. The drug use frequency variable reflected how many days drugs were used among drug users in the past 90 days.

Lastly, research aim 3 required testing for moderation. Moderation can be defined as the "effect of a third variable or construct changing the relationship between two related variables/constructs. That is, a moderator indicates that the relationship between variables changes based on the amount of another variable added to the model" (Hair et al., 2006).

Preliminary steps in preparing the data for testing moderation effects were conducted as described in Frazier, Tix \& Barron (2004). As such, the first order predictors and moderator terms were mean centered. It is recommended that continuous variables be centered when conducting moderation analyses because the predictor and moderator variables are highly correlated with the product terms created for them. This can create instability in the values of the estimated regression weights (Aiken \& West, 1991; Cohen, Cohen, West, \& Aiken, 2003; Jaccard, Turrisi, \& Wan, 1990). Several authors have shown that if the first order variables are mean centered (i.e., transformed from the raw score to a deviation score scaling by subtracting the variable mean from all observations) the resulting product term will be minimally correlated or uncorrelated with 
the first order variables if the variables are more or less bivariate normal (Little, Card, Bovaird, Preacher, \& Crandall, 2007).

Next, product terms were created between the predictor and moderator terms representing their interaction. Finally, the interpretation of significant moderation effects consisted of a two-step process. Step one included calculating the predicted values of the outcome variables for each representative group, such as those who score at the mean, 1 standard deviation above and 1 standard deviation below the mean on the predictor and moderator variables (Aiken \& West, 1991; Cohen et al., 2003). These predicted values were then used to create a graphical representation summarizing the form of the moderator effect (Frazier, Tix and Barron, 2004). 


\section{CHAPTER IV. RESULTS}

This study aimed to examine the influence of acculturative stress on substance use and HIV-risk behaviors among recent Latino immigrants, and to find whether religious coping mechanisms had a moderating effect on that relationship. The data analytic plan for the present study consisted of preliminary analyses that included: 1) obtaining descriptive statistics of the sample, 2) missing data analyses, 3) checks for non-normality, 4) testing for the potential influence of covariates in the model, 5) conducting confirmatory factor analysis to examine each of the measurement models, and 6) testing for measurement invariance over time between pre and post immigration measurement models. The primary analyses also consisted of testing the overall fit of the structural models as well as its path coefficients and standard errors. Zero-inflated Poisson (ZIP) modeling was used with the drug use behavior outcome and analyzed through multivariate Poisson regression. Finally, interaction effects were analyzed and interpretation was aided with the use of graphical representation summarizing the form of the moderator effect.

\section{Preliminary Analyses}

Several analytic procedures and techniques were employed to determine if there were outliers, missing data, or non-normality. A review of the results of these preliminary analyses is outlined below.

\section{Descriptive statistics}

Table 4 presents means and standard deviations for all continuous study variables. Categorical variables are summarized in terms of proportions. For descriptive purposes the drug use variable was dichotomized due to the small amount participants who 
reported engaging in this behavior. The dichotomized variables were created by recoding the response scale for each behavior so that 0 was coded as "No" (i.e., no use in the last 90 days) and 1 was coded as "Yes" (i.e., any valid response other than zero, reflecting use in the 90 days). The non-normal distribution of the frequency of drug use variable was left untransformed in the main analysis as this variable was used only in the zero-inflated Poisson modeling analyses that assumed non-normality.

\section{Missing data}

There were small amounts of missing data (no more than 2 percent $(n=7)$ on a given variable). Furthermore, missing data were accommodated through SEM by employing full information maximum likelihood (FIML) missing data methodology. MPLUS, as well as other structural equation modeling software offer this full information maximum likelihood solution to missing values where all available observed information is used to produce the maximum likelihood estimation of parameters (Wothke, 2000).

\section{Non-normality}

Traditional maximum likelihood methods of SEM assume that the continuous variables in the model are multivariately normally distributed. According to Kline (2005), continuous variables were deemed non-normal if they yielded absolute skewness and kurtosis values greater than 3.0 and 8.0 respectively. Frequencies including skewness and kurtosis indices are displayed in Table 5. A positively skewed distribution was found for alcohol use quantity $($ skew $=4.06$, kurtosis $=25.52)$, alcohol use frequency $($ skew $=2.93$, kurtosis $=11.65)$, and income $($ skew $=3.76$, kurtosis $=20.44)$. A square root data transformation was used to arrive at an approximately normal distribution for these variables. The transformed values were used in subsequent analyses. Due to the 
highly skewed drug use outcome variable, non-normality was evident. As discussed in the previous chapter, a zero-inflated Poisson analyses was conducted to accommodate this non-normality.

\section{Correlational analyses}

Table 6 displays bivariate correlations for the key observed variables considered for use in the present study. Pearson product-moment correlation coefficients were calculated to determine the relationship among continuous variables. Spearman rho correlation coefficients were calculated for dichotomous variables.

Results indicated that multicollinearity existed between the internal and positive religious coping predictor variables $(r=.89, p<.01)$. Multicollinearity occurs when predictor variables are so highly correlated (usually above .80 ) that obtaining reliable estimates of their individual regression coefficients becomes problematic (Grewal, Cote \& Baumgartner, 2004). This is due to the assumption that that the two highly correlated variables are essentially measuring the same construct and thus conveying the same information in a single analysis. Multicollinearity has been documented to lead to inaccurate estimates of coefficients and standard errors as well as inference errors (Kline, 2005).

Due to the multicollinearity between internal and positive religious coping, it became necessary to remove one of variables from the analyses. Unlike the Internal Religious Coping Subscale of the WORCS, the Positive Religious Coping Subscale of the Brief RCOPE has been validated with Latino populations (Gonzalez-Morkos, 2005). Thus, a decision was made to remove internal religious coping from subsequent analyses. 


\section{Covariate analyses}

Statistical tests were conducted to assess the potential influence of covariates in the research model. An analysis of variance (ANOVA) and Pearson's correlation was used to evaluate the potential influence of education and income respectively. Each variable was evaluated for its influence on the key observed variables (internal, external, positive and negative religious coping, acculturative stress, alcohol quantity, alcohol frequency, harmful/hazardous alcohol use, drug use incidence, and condom use during oral, vaginal and anal sex). Spearman's rho correlation analyses were conducted on the binary covariates (gender and documentation status) for the previously mentioned key observed variables.

Results from the one-way ANOVA indicated that participants with less education used all religious coping styles more frequently than those with higher education (internal religious coping $F(4,414)=2.94, p=.02, \eta^{2}=.03$, external religious coping $F(4,414)=$ $5.19, p=<.001, \eta^{2}=.05$, positive religious coping $F(4,414)=2.68, p=.03, \eta^{2}=.03$ and negative religious coping $\left.F(4,414)=3.92, p=.004, \eta^{2}=.04\right)$. Higher harmful/hazardous drinking patterns, as assessed by the AUDIT, were also found among participants with less education $F(4,414)=3.55, p=.007, \eta^{2}=.03$.

Pearson's correlation analyses indicated a higher frequency of alcohol use among participants with higher income $(r=.19, p<.001)$. Participants reporting higher income also had higher levels of HIV risk as indicated by lower condom use when engaging in oral $(r=.11, p<.05)$ and vaginal $\operatorname{sex}(r=.11, p<.05)$.

Spearman's Rho correlations indicated a statistically significant correlation between gender and religious coping with women reporting higher rates of internal 
religious coping $(r=-.22, p<.001)$, external religious coping $(r=-.14, p=.006)$, and positive religious coping $(r=-.18, p<.001)$ in relation to their male counterparts. Males reported higher rates in quantity $(r=.31, p<.001)$ and frequency of alcohol consumption $(r=.25, p<.001)$, as well as harmful/hazardous alcohol use behavior $(r=.28, p<.001)$. No significant gender differences were found in sexual HIV risk behaviors (condom use). In regard to documentation status, undocumented Latino immigrants reported higher rates of engaging in drug use $(r=-.14, p<.001)$ while documented immigrants reported more frequency of alcohol consumption $(r=.11, p<.05)$. Significant correlations between documentation status and religious coping styles were also found with undocumented immigrants reporting higher levels in all religious coping styles [internal religious coping $(r=-.25, p<.001)$, external religious $(r=-.20, p<.001)$, positive religious coping $(r=-.25, p<.001)$ and negative religious coping $(r=-.26$, $p<.001)$, in comparison to documented immigrants. Differences in condom use across

documentation status were also found with documented immigrants reporting higher rates of condom use during oral $(r=.19, p=.001)$ and anal $\operatorname{sex}(r=.20, p=.01)$ than undocumented immigrants.

\section{Measurement models}

Confirmatory factor analyses (CFA) was used to test and examine if hypothesized indicators adequately represented all of the latent constructs in the structural model (preimmigration and post immigration alcohol use and pre and post-immigration condom use.) The measurement models were just identified. This type of fit occurs when the number of free parameters exactly equals the number of known values; therefore, it is a 
model with zero degrees of freedom and therefore has non-interpretable fit indices $\left(\chi^{2} p\right.$ value $=.000, \mathrm{CFI}=1.00, \mathrm{RMSEA}=0.00)$.

For pre-immigration alcohol use index, the factor loadings suggested that the three indicators loaded strongly onto the latent construct ( $\beta$ estimates ranging from .81 to .89) (See Figure 1). Eighty percent of variability in alcohol use index was explained by the harmful/hazardous alcohol use indicator. Seventy-five percent was explained by quantity of alcohol use. Sixty-five percent of variability was explained by frequency of alcohol use.

As presented in Figure 2, for post-immigration alcohol use index the factor also loaded strongly onto the latent construct ( $\beta$ estimates ranging from .82 to .87 ). Seventyfive percent of variability in the alcohol use index latent variable was explained by the harmful hazardous alcohol use indicator. Seventy-four percent was explained by quantity of alcohol use. Sixty-eight percent of variability was explained by frequency of alcohol use.

The factors for the pre-immigration condom use also loaded strongly onto the latent construct ( $\beta$ estimates ranging from .69 to .91) (See Figure 3). Forty-eight percent of variability in condom use was explained by condom use during oral sex. Seventy-one percent was explained by condom use during vaginal sex. Eighty-three percent of variability in condom use was explained by condom use during anal sex.

As shown in Figure 4, the factors for the post-immigration condom use also loaded strongly onto the latent construct ( $\beta$ estimates ranging from .67 to .90). Forty-five percent of variability in condom use was explained by condom use during oral sex. 
Seventy-two percent was explained by condom use during vaginal sex. Eighty-one percent of variability in condom use was explained by condom use during anal sex.

\section{Measurement invariance}

The measurement model testing for configural invariance across the pre and postimmigration alcohol use index latent construct had adequate model fit well in accordance to the fit index criteria described in the previous chapter $\chi^{2}(5,415)=20.56, p<.01$, RMSEA $=.087, \mathrm{CFI}=.99, \mathrm{SRMR}=.03$. Metric invariance of the factor loadings between the two time points was tested by constraining the factor loadings between the two groups. This model did not result in a significant difference in fit from the configural model that placed no constraints on the parameters, $\chi^{2}(7,415)=26.31, p<.001$, RMSEA $=.082, \mathrm{CFI}=.99, \mathrm{SRMR}=.03$. When comparing the constrained and unconstrained models, no significant differences were found $\Delta \chi^{2}=5.76, \Delta d f=2, \Delta \mathrm{CFI}=$ $.002, p>.05$.

The unconstrained measurement model testing for configural invariance across the pre and post immigration condom use latent constructs indicated good fit, $\chi^{2}(8,415)=$ $14.48, p=.07, \mathrm{RMSEA}=.05, \mathrm{CFI}=.99, \mathrm{SRMR}=.02$. The measurement model testing for metric invariance by constraining the factor loadings between the two groups did not results in a significant difference in fit over the configural model, $\chi^{2}(10,415)=16.41, p$ $=.09, \mathrm{RMSEA}=.16, \mathrm{CFI}=.99, \mathrm{SRMR}=.03$. No significant differences were found between the constrained and unconstrained model $\Delta \chi^{2}=1.93, \Delta d f=2, \Delta \mathrm{CFI}=.00$, $p>.05$. 


\section{Primary Analyses}

After preliminary analyses were conducted, it was determined that there were inconsequential amounts of missing data and that issues of non-normality could be adequately accommodated. Furthermore, the influence of covariates, the quality of the latent constructs and their measurement invariance were confirmed. Therefore, the primary analytic approach which involved the use of structural equation modeling (SEM) and zero-inflated Poisson modeling using the MPlus software (version 6.0) was pursued. Below is a review of the research aims and hypotheses, model fit criteria and evaluation of hypotheses and results.

Research Aim 1 was to determine the relationship of acculturative stress to substance use and HIV risk behaviors of recent Latino immigrants.

Research Aim 1- Hypothesis 1a: Latino immigrants with higher acculturative stress will have higher rates of alcohol use and HIV-risk behaviors than recent Latino immigrants with lower acculturative stress. An implied structural model represented by the Aim 1 paths of Figure 5 was used to test this hypothesis. Pre-immigration alcohol use and pre-immigration HIV-risk (condom use) were controlled for in this model. Gender, immigration status, income and education were also included as covariates. The implied structural model indicated a good fit $\chi^{2}(91,415)=217.31, p<.001$, RMSEA $=.06$, $\mathrm{CFI}=.95, \mathrm{SRMR}=.05$. Results indicated no significant relationship between acculturative stress and alcohol use/HIV risk (condom use). Inspection of the residuals and modification indices revealed no theoretically meaningful points of ill fit in the model. Figure 7 presents the parameter estimates for the structural coefficients. 
Latino immigrant males had a higher frequency of post immigration alcohol use behaviors in comparison to their Latina counterparts $(\beta=.11, p=.02)$. Statistically significant positive relationships were found between pre and post immigration alcohol use $(\beta=.55, p<.001)$ and pre and post immigration condom use $(\beta=.23, p<.001)$. Thus, higher rates of alcohol and HIV risk behavior prior to coming to the U.S. were associated with higher rates of these behavior post immigration. Participants who used more alcohol at pre-immigration also reported more pre-immigration HIV risk behaviors $(r=.15, \mathrm{p}<.01)$. Pre-immigration alcohol use was also significantly correlated with gender with males using more alcohol at pre-immigration $(r=.36, \mathrm{p}<.001)$.

\section{Research Aim 1- Hypothesis 1b: Latino immigrants with higher acculturative} stress will have higher rates of drug use than recent Latino immigrants with lower acculturative stress. This hypothesis was tested through ZIP modeling in a multivariate Poisson regression analysis. As seen in Figure 8 the model analyzed the influence of acculturative stress on incidence of drug use (dichotomous variable: yes/no) separately from the influence of acculturative stress on frequency of drug use among drug users. Gender, education, income, immigration status, and pre-immigration drug use were controlled for in the model. In ZIP models, path coefficients for the dichotomous variable (drug use incidence) are interpreted as odds ratios (OR), which represent the multiplicative increase/decrease in the odds of event occurrence with each $1 S D$ increase in acculturative stress. Path coefficients for count variables (frequency of drug use among drug users) are interpreted as incident rate ratios (IRR), which represent the multiplicative increase in the expected count with each $1 S D$ increase in the acculturative stress variable (Schwartz et al, 2011). 
Results indicated no significant relationship between acculturative stress and incidence of drug use (see Figure 8). There was a significant inverse relationship between acculturative stress and drug use frequency. Results suggest that each standard deviation increase in acculturative stress was associated with a $43 \%$ decrease in frequency of drug use among users $(\operatorname{IRR}=.57, p<.01)$.

Participants that reported engaging in pre-immigration drug use were also 1.34 times more likely to engage in drug use post immigration $(\mathrm{OR}=1.34, p<.001)$. Having an undocumented immigration status was associated with $18 \%$ decrease in risk of engaging in post immigration drug use $(\mathrm{OR}=.82, p=.01)$. The frequency of post immigration drug use was also 1.95 times higher among documented users than among undocumented users $(\operatorname{IRR}=1.95, p<.001)$. Results indicated that each standard deviation increase in education was associated with a $49 \%$ decrease in the frequency of drug use among users $(\operatorname{IRR}=.51, p<.001)$.

For this research aim it was hypothesized that a positive relationship existed between acculturative stress and drug use, thus the model was not consistent with the hypothesis.

Research Aim 2 was to determine the relationship of specific religious coping mechanisms to the acculturative stress experienced by recent Latino immigrants. Hypothesis 2a: Latino immigrants who use internal, external, and positive religious coping at higher rates will experience lower acculturative stress. Hypothesis $2 b$ : Latino immigrants who use negative religious coping at higher rates will experience higher acculturative stress. Research Aim 2 with both accompanying hypothesis (Hypothesis 2a and $2 \mathrm{~b}$ ) were tested through a path model analysis represented by Aim 2 of Figure 5 . 
Gender, immigration status, income and education were included as covariates. The implied model was just identified and as such no fit indices are available. As seen in Figure 7, an examination of the standardized parameter estimates revealed no significant relationships between any of the religious coping mechanisms and acculturative stress. Thus, hypothesis $2 \mathrm{a}$ and $2 \mathrm{~b}$ were inconsistent with the results of the analysis.

An examination of the covariates indicated that undocumented Latino immigrants had higher rates of external religious coping $(\beta=-.13, p<.01)$, positive religious coping $(\beta=-.26, p<.001)$, and negative religious coping $(\beta=-.23, p<.001)$, in comparison to their documented counterparts. Latina immigrant women also had higher rates of positive religious coping $(\beta=-.20, p<.001)$ in comparison to Latino immigrant males. No gender differences were found in the use of external and negative religious coping. There was also no difference across income or education levels in use of religious coping mechanism, or in acculturative stress across any of the covariates (gender, immigration status, income and education).

Research Aim 3 was to identify whether religious coping mechanisms moderate the relationship between acculturative stress and alcohol abuse/HIV risk behaviors among recent Latino immigrants.

Research Aim 3- Hypothesis 3a: The relationship between acculturative stress and alcohol use/HIV-risk behavior will be weaker among Latinos who report using more, external and positive religious coping. An implied structural model represented by the Aim 3 paths of Figure 5 was used to test this hypothesis. Gender, immigration status, income, education, pre-immigration alcohol use and pre-immigration condom use were included as covariates in the model. As described in Chapter 3, the predictor 
(acculturative stress) and moderator (external and positive negative coping) variable terms were mean centered. The literature also suggests that any continuous covariates be mean centered prior to examining interaction effects (Frazier \& Tix, 2004). As such, the education and income variable were mean centered prior to conducting the analysis. Next, product terms were created between the predictor and moderator terms representing their interaction (the product of acculturative stress with external religious coping and acculturative stress with positive religious coping). Unlike the previous analyses conducted for Research Aims 1 and 2, results for the moderation models require interpretation of the unstandardized (B) rather than the standardized ( $\beta$ ) regression coefficients. This is because in equations that include interaction terms, the $\beta$ coefficients for these terms are not properly standardized, and thus not interpretable (Aiken \& West, 1991; West et al., 1996). Although the model indicated good fit $\left(\chi^{2} 163,415\right)=313.77$, $p<.001, \mathrm{RMSEA}=.05, \mathrm{CFI}=.95, \mathrm{SRMR}=.05$, no interaction effects for external or positive religious coping were found (see Figure 7). Thus, findings from the analysis were not consistent with the hypothesis.

Research Aim 3- Hypothesis 3b: The relationship between acculturative stress and alcohol use/HIV-risk behavior will be stronger among Latinos who report using more negative religious coping. This hypothesis was tested simultaneously in the structural model described above in Hypothesis 3a. As seen in Figure 7, no interaction effects between acculturative stress and negative religious coping were found on condom use. There was, however, a statistically significant interaction between acculturative stress and negative religious coping on alcohol use $(\mathrm{B}=-.18, p=.04)$. Figure 9 presents a plot of this moderation effect following the method and online computational plotting 
approach of Dawson and Richter (2006). The plot illustrates that participants' reporting high levels of negative religious coping reported more alcohol use when they experienced low levels of acculturative stress. Conversely, participants reporting low levels of negative religious coping reported less alcohol use when they experienced low levels of acculturative stress. Negative religious coping did strengthen the relationship between acculturative stress and alcohol use, albeit, in an inverse direction, thus the implied structural model was consistent with the hypothesis.

Research Aim 3-Hypothesis 3c: The relationship between acculturative stress and drug use will be weaker among Latinos who report using more, external and positive religious coping. This interaction effect was tested using ZIP modeling in a multivariate Poisson regression analysis. Therefore, interaction effects between each moderator were tested separately for incidence of drug use and drug use frequency. As exhibited in Figure 8, no significant interaction effects were found between acculturative stress with external or positive religious coping for incidence or frequency of drug use. Findings from this analysis do not support the proposed hypothesis.

\section{Research Aim 3-Hypothesis 3d: The relationship between acculturative stress} and drug use will be stronger among Latinos who report using more negative religious coping. This interaction effect was tested simultaneously in the ZIP model described above in Hypothesis 3c. Findings indicated a statistically significant interaction between acculturative stress and negative religious coping on frequency of drug use among drug users $(\mathrm{B}=-.18, p=.01)$. Figure 10 displays a plot of this moderation effect following the approach of Dawson and Richter (2006). The plot illustrates that participants reporting 
high levels of negative religious coping reported more frequent drug use when they experienced low levels of acculturative stress.

\section{Supplemental analyses}

Supplemental analyses were conducted to examine any direct effects of religious coping on the outcome variables (alcohol use, condom use, and drug use). Figure 11 presents the structural model testing the association between of external, positive and negative religious coping on alcohol use and condom use. As with the previous models, pre-immigration alcohol use and pre-immigration condom use were controlled for in this model. Gender, immigration status, income and education were also included as covariates, as previous analyses indicated an influence of these variables on the predictor and/or outcome variables in the model. Fit indices indicated good model fit $\chi^{2}(107,415)$ $=231.32, p<.001, \mathrm{RMSEA}=.05, \mathrm{CFI}=.96, \mathrm{SRMR}=.05$. Further examination of the path coefficients in the model indicated that participants with higher levels of external $(\beta=-.20, p<.01)$, and positive religious coping $(\beta=-.14, p=.03)$, reported lower scores

on the alcohol use index. No significant relationships were found between negative religious coping and alcohol use or between any religious coping mechanism and condom use.

Figure 12 presents a zero-inflated Poisson model used to test the influence of external, positive and negative religious coping on incidence and frequency of drug use. Gender, immigration status, income, education and pre-immigration drug use were included as covariates in the model. Results suggest that each standard deviation increase in external religious coping was associated with a $22 \%$ decrease in the risk of engaging in 
drug use $(\mathrm{OR}=.79, p=.03)$. Each standard deviation increase in negative religious coping was associated with a $20 \%$ increase in the risk of engaging in drug use $(\mathrm{OR}=1.20$, $p=.01$ ). For each standard deviation increase in negative religious coping frequency of drug use among drug users also increased by $49 \%(\operatorname{IRR}=1.49, p=.02)$. No relationships were found between positive religious coping and drug use. 


\section{CHAPTER V. DISCUSSION}

\section{Summary of Dissertation Findings}

The present study examined the influence of acculturative stress on the substance use and HIV risk behaviors of recent Latino immigrants and whether religious coping had a moderating effect on that relationship. Three specific aims were tested. The first aim of the study was to determine the relationship of acculturative stress to the substance use/HIV risk behaviors of recent Latino immigrants. It was hypothesized that Latino immigrants with higher rates of acculturative stress would have higher rates of substance use and HIV-risk behaviors. There was no support for this hypothesis. The second aim examined the relationship between religious coping mechanisms (external, positive and negative religious coping) and the acculturative stress experienced by this population. No associations were found between acculturative stress and any of the religious coping mechanisms. Aim three was to identify whether any of the religious coping mechanisms moderated the relationship between acculturative stress and substance use/HIV risk behaviors. Only negative religious coping appeared to have a moderating effect between acculturative stress and substance use/HIV risk behaviors. Higher rates of negative religious coping strengthened an inverse relationship between acculturative stress and alcohol use. Participants with low levels of negative religious coping reported more alcohol use when they experienced higher levels of acculturative stress. Conversely, participants reporting high negative religious coping reported lower alcohol use when they experienced higher levels of acculturative stress. Negative religious coping also had a moderating effect on an inverse relationship between acculturative stress and frequency of drug use among drug users. Thus, drug using participants with high levels of negative 
religious coping reported less frequent drug use when they experienced higher levels of acculturative stress. Supplemental data analyses revealed lower alcohol use among participants reporting higher levels of external and positive religious coping. Higher levels of external religious coping were associated with a decreased risk of engaging in drug use. Higher levels of negative religious coping were associated with increased incidence and frequency of drug use.

\section{Associations between negative religious coping and substance use.}

According to Pargament (2002b), negative religious coping styles emphasize passive or deferential responses to stress were difficulties are viewed as punishment or abandonment from a higher being (God). The literature has linked this form of religious coping to feelings of guilt, shame, and overall poor physical and mental health outcomes (Chatters, 2000; Pargament 2002b). In the present study, participants with high rates of negative religious coping reported less alcohol and drug use when they experienced high levels of acculturative stress. As such, current results suggest that this coping style may play a protective role in the risk behaviors of recent Latino immigrants experiencing acculturative stress.

This finding may be partially explained by a well-documented cultural concept among Latinos known as fatalismo (fatalism). Fatalismo refers to the belief that events occur due to luck, fate, or powers beyond the person's control (Antsel, 2002; Moreno, 2007). Among Latinos, it is not uncommon for religion to play a role in fatalismo, where negative expectations are combined with a relinquishment of power to God (Castro, Cota \& Vega, 1999). In this manner, adverse life circumstances such as illness are viewed as part of God's will (Interian \& Diaz-Martinez, 2007). Fatalismo and negative religious 
coping therefore are similar in nature in that they come from an external locus of control perspective that view negative experiences as the will of God.

The literature predominantly cites fatalismo as a risk factor that keeps Latinos from engaging in various health promotion and disease detection behaviors (Castro, Cota \& Vega, 1999; Otero-Sabogal, Stewart, Sabogal, Brown, Pérez-Stable, 2003; Smiley, McMillan, Johnson \& Ojeda, 2000). Yet evidence of the negative health effects of fatalismo still remain inconclusive, as other studies have suggested that it may play a beneficial role in the health behaviors of Latinos (Larkey, Hecht, Miller \& Alatorre, 2001; Neff \& Hoppe, 1993). Larkey and colleagues (2001) conducted focus groups with Latinas being screened for breast and cervical cancer as part of a study investigating the influence of cultural norms on the health seeking behaviors of Latinas. Data from the focus groups suggested that fear, as a component of fatalismo, actually supported health seeking behaviors among participants. Among the author's recommendations was that prevention service delivery for more traditional Latinos include:

Public health campaigns promoting screening visits and teaching about warning signs should couple knowledge with fear presentations with acknowledgement of the role that spirituality plays in the lives of the target population, and imply a partnership between God's divine help and God's earthly helpers-health professionals-in the maintenance of health (i.e., the health of the body, the temple of the Holy Spirit, as described by one participants.) (p. 76)

In this study, it is possible that fear, too, may have played a role in the protective influence of negative religious coping on the alcohol and drug use behaviors of the recent Latino immigrants. In the presence of highly stressful circumstances, these individuals 
may have deferred from engaging in the aforementioned behaviors for fear of further Godly repercussions.

Although present study findings suggests negative religious coping may have a protective moderating influence on substance use behaviors during highly stressful situations, supplemental analyses indicated higher rates in the incidence and frequency of drug use among participants who utilized more negative religious coping. Further investigations are necessary in order to tease apart the elements of fatalismo and in particular negative religious coping that may be beneficial and detrimental to health behaviors among Latinos.

Previously, researchers have agreed that there is a need for further knowledge in this area. For instance, in a commentary regarding fatalismo and its role in health-related research and practice with Latinos, Abraido-Lanza and colleagues (2007) call into question the negative impact that fatalismo has on cancer screening among Latinos. The authors highlight conceptual and methodological problems associated with studies of fatalismo among Latinos and call for future research to better understand the interconnection between fatalismo and spiritual attributions in this population (AbraidoLanza, Viladrich, Florez, Cespedes, Aguirre, \& De La Cruz, 2007).

\section{Associations between external/positive religious coping and substance use.}

Findings from this investigation indicated that Latino immigrants who used more external and positive religious coping engaged in less alcohol use behaviors and were at less risk of engaging in drug use. These results are consistent with previous literature that external and positive religious coping can serve as a protective factor against substance use behaviors. Results also indicated that Latina immigrant women used positive 
religious coping more frequently than their male counterparts. Undocumented Latino immigrants used both external and positive religious coping more frequently than documented immigrants. These findings also fall in line with previous research were these coping styles have been found to be more prominent among vulnerable populations such as the elderly (Pargament et al., 2001) and other ethnic minorities (Ellison et al., 2009). Outcomes from the present study could inform research as to the possible benefits of fostering the use of existing religious coping styles in alcohol use prevention and intervention programs with these populations.

\section{Acculturative stress and religious coping}

No relationship was found between acculturative stress and any of the religious coping mechanisms. It may be religious coping is not a particularly beneficial coping styles in dealing with the stressors involved in the immigration process. Another possibility is that these coping mechanisms function differently across religious affiliations in this sample. Previous research has found varying outcomes on the use of religious coping across religious denominations. Because various religious affiliations place emphasis on different beliefs, using religion as a coping mechanism may lead to dissimilar outcomes for individuals in diverse religious affiliations (Alferi et al., 1999; Park, Cohen, \& Herb, 1990). Alferi \& colleagues (1999) conducted an investigation on religious coping among Latinas with early-stage breast cancer. The study found that Latina women who identified as Catholic and attended church regularly at six months post-surgery predicted greater distress at 12 months post-surgery. Conversely, Latinas who identified as Evangelical and reported obtaining emotional support from church members at six months post- surgery predicted less distress at 12 months after surgery 
(Alferi et al., 1999). Another study by Park and colleagues (1990) found denominational differences across religious coping depending on whether the stressor was viewed as controllable or uncontrollable. When Catholics and Protestants experienced a controllable stressor, religious coping appeared to reduce distress for Catholics, and increase distress for Protestants. On the other hand, when a stressor is uncontrollable, religious coping increased distress for Catholics but decreased distress for Protestants. The authors concluded that the emphasis of guilt and forgiveness of guilt in Catholic ideology better prepares devotees for controllable stressors while the emphasis of faith and acceptance in Protestant religions better prepares devotees for uncontrollable stressors (Park, Cohen, \& Herb, 1990). In this manner, it may be that religious coping influences acculturative stress differently across religious affiliations among Latino immigrants. However, there is no evidence to support this speculation as data on religious denomination was not collected in the present study. Future studies should look at associations between religious coping and acculturative stress across religious affiliation in order to uncover any differences that may exist.

\section{Associations between documentation status and drug use behaviors.}

Unexpectedly, study findings suggested that both the drug use incidence, as well as frequency of drug use among users, was lower in undocumented immigrants when compared to their documented counterparts. Furthermore, higher rates of acculturative stress were associated with a lower frequency of drug use among users. It may be that factors related to social control played a part in the decreased drug use rates among undocumented Latino immigrants in this sample (De La Rosa, Sanchez, Dillon, Blackson, Ruffin, \& Schwartz, 2011). As a result, undocumented immigrants may be 
more cognizant of, and invested in not drawing attention to themselves for drug related behaviors. For individuals who are willing to go through the stressors involved in illegal immigration, the desire to engage in drug use may be curbed not only by fear of legal repercussions but by prospect of new beginnings in the host country.

\section{Implications for Social Work}

The social work profession recognizes immigration as a complex social, cultural, and political process (NASW, 2010). Social workers have historically been instrumental in assisting immigrants' transition into American society. Amid the current political context of U.S. immigration policy and as the number of Latinos residing in the United States grows, the need to assist these individuals in making smooth transitions into American society is increasingly imperative. Religion represents a valuable cultural resource for many Latino immigrants who are struggling with the loss of a homeland and separation from family and friends. As social workers, it is important to evaluate to what degree these resources may be accessed as a means of assisting immigrants in coping with the strains of the acculturation process. It is hoped that findings from this study can help guide future directions in micro, meso, and macro-level social work practice.

\section{Micro-level social work practice implications}

Despite growing numbers of Latino's immigrating to the United States, challenges in understanding the service needs of these immigrants have never been greater (Alegria et al., 2006). It is necessary for social workers to improve their skills and strategies in assisting recent Latino immigrants in managing the negative aspects of the acculturation process. Furthermore, service delivery of substance abuse and HIV risk prevention/interventions programs with Latinos has generally been based on service 
models and information used with non-Latino White males (Alegria et al., 2006). Few behavioral intervention and treatments have been specifically developed for Latino immigrants (Wagner, 2003). As a consequence, there is insufficient empirical research to inform service providers of the appropriate issues to target in programs with this population (Alegria et al., 2008).

Greater attention to Latino cultural values in substance abuse/HIV risk interventions have been an ever-increasing development in the literature (Alvarez et al., 2007). Available evidence of specific Latino cultural values that may play a role in these behavioral outcomes include: a) dignity (dignidad), b) respect (respeto), c) reliance on the support of extended family (familismo), d) interpersonal relationships (personalismo), e) downplaying conflict (simpatia), f) strict gender roles (machismo, marianismo) (Kail \& Elberth, 2003; Gil \&Vega, 2001; Waters, Fazio, Hernandez, \& Segarra, 2002). Yet little is known about how religious coping as a cultural value influences these behavioral outcomes among recent Latino immigrants. In the present study external and positive religious coping were inversely associated with the substance use behaviors of recent Latino immigrants. Negative religious coping also appeared to have a protective influence on substance use in the sample. As such, micro-level social work practice implications of the current research include the possible benefits of incorporating these cultural values into substance use intervention and prevention programs with Latinos. In the present study external and positive religious coping were inversely associated with the substance use behaviors of recent Latino immigrants. Negative religious coping also appeared to have a protective influence on substance use in the sample. As such, microlevel social work practice implications of the current research include investigating the 
possible benefits of integrating these cultural values into substance use intervention/prevention programs with Latinos.

Service delivery implications also include linking recent immigrants who have used external religious coping resources in their country of origin to similar religious centers in the host country. These resources may provide Latino immigrants with social support, which could prove to have a protective influence against acculturative stress along with all of its negative outcomes (Finch \& Vega, 2003; Vega \& Amaro, 1994). Furthermore, these centers of worship may provide familiarity to Latino immigrants entering into a new culture and environment as well as a means to accessing tangible services such as counseling, legal aid, and English and Spanish literacy programs. Religious centers may be viewed as a safe haven for immigrants who are struggling to adjust to a new society with a different language and customs (Pew Research Center, 2009). Social workers should assess recent Latino immigrants' past and present social and cultural resources as well as coping strategies, and facilitate connections to religious resources if and when appropriate.

\section{Meso level social work implications}

Present study findings suggest that external religious coping styles, which rely heavily on the social support of a religious community, were associated with less substance use behaviors among recent Latino immigrants. In this manner, fostering effective partnerships between the social work and religious communities may lead to beneficial physical and mental health outcomes among Latino immigrants. Religious institutions have a long-standing history in the provision of social services (Cnaan, Wineburg, \& Boddie, 1999). Yet, there exists noticeable reluctance in developing 
collaborations between secular and religious-based social services. According to Cnaan and colleagues (1999) the social work profession has demonstrated:

A widespread oversight regarding the role that religious organizations and congregations played in the provision of social services. Social work may have had to separate from its religious roots to gain legitimacy as a profession. Yet social work scholars and educators have given little attention either to the extraordinary role of religion in the provision of social services or to its considerable influence on social service development...If the field does not know where it has been how will it know where it is going?" (p. 1)

This hesitation may be due to the fact that religious institutions often have conflicting values with that of the social work profession (i.e., abortion, homosexuality, etc.). Nevertheless, social workers and religious leaders may be able to effectively work together in mediating the stressors involved in acculturation process of recent Latino immigrants. Reconnecting the profession with the religious community can take the form of interdisciplinary teams working together with religious community leaders in capacity building among centers of worship in immigrant receiving communities. These partnerships can lead to the development faith-based outreach teams, pastoral counseling, substance abuse and HIV outpatient treatment and recovery support, as well as risk reduction interventions.

\section{Macro level social work implications}

U.S. policies are currently in place that recognizes the importance of taking into account cultural differences as well as spiritual perspectives in the service delivery process. For instance, the nation's predominant health care accrediting body, the Joint 
Commission on Accreditation of Healthcare Organizations (JCAHO) requires the administration of a spiritual assessment (Hodge, 2006). According to JCAHO, a spiritual assessment should at a minimum:

Determine the patient's denomination, beliefs, and what spiritual practices are important to the patient. This information would assist in determining the impact of spirituality, if any, on the care/services being provided and will identify if any further assessment is needed. The standards require organizations to define the content and scope of spiritual and other assessments and the qualifications of the individual(s) performing the assessment [with many organizations requiring chaplains to be board certified]. (JCAHO, 2011).

Although spirituality and religion are essentially viewed as distinct constructs, religion is often a central focus of spirituality (Canda \& Furman, 1999). According to Miller (1998) spirituality is a personal attribute that involves a search for meaning and a relationship with that which is sacred to the individual. Religion refers to organized structures that center around particular beliefs, behaviors, rituals, ceremonies, and traditions (Canda \& Furman, 1999). Historically, spirituality and religion are considerably interwoven. Religion has traditionally been regarded as encompassing the individual as well as the institutional, the functional and experiential. Within this perspective, one can conceptualize a private (personal), as well as, public (institutional) form of religiousness. It is here where the overlap between religion and spirituality are more apparent (Miller \& Thoresen, 2003). Furthermore, the overlap between religion and spirituality are subjective to the individual. For some persons, there may be considerable overlap between their religiousness with spirituality. Others, however, even 
within the same religion, there may have little overlap (Miller \& Thoresen, 2003). Thus spirituality and religion may be best described as overlapping constructs, that while sharing some characteristics, retain distinctive features of their own (Miller \& Thoresen, 2003).

Little empirical evidence exists suggesting that individuals experience actual antagonisms between their spiritual beliefs and religious practices (Shorkey, Uebel, \& Windsor, 2008). Research indicates that most people experience spirituality within an organized religious context and do not perceive distinctions between the two (Marler \& Hadaway, 2002; Zinnbauer et al., 1997). Zinnabauer and colleagues (1997) sampled 346 people in Pennsylvania and Ohio. Participants completed several questionnaires about perceived similarities and differences between religiousness and spirituality, as well as scales covering beliefs and attitudes about God, oneself, and others. The sample consisted of individuals from a wide range of demographic and religious/spiritual backgrounds and found that approximately $74 \%$ described themselves as spiritual and religious (Zinnbauer et al. 1997).

In lieu of such findings, it appears probable that spiritual assessments have the potential to uncover religious coping styles that can in turn be used a resource during difficult life circumstances. From an organizational perspective, social workers can advocate for broader integration of spiritual assessments within social service agencies. These spiritual assessments have the potential to provide social workers with a wealth of information regarding a client's belief system. For instance, integrating spiritual assessments into substance abuse programs can assist clinicians in the incorporation of a client's belief systems into their treatment. It should also be noted that when assessing 
the belief systems of Latino's, social workers should ensure that these assessment care completed in a culturally sensitive manner.

Furthermore, social workers can encourage their agencies to hold educational inservice trainings on a broad array of spiritual and religious perspectives. This can be a fundamental step towards having clinicians that are sensitive to the spiritual and religious needs of their clients. Miller (1999) recommends these trainings incorporate an emphasis on a) a non-judgmental accepting and empathetic relationship with the client, b) an openness and willingness to take time to understand the client's spirituality as it may relate to mental health-related issues, c) some familiarity with culturally related values, beliefs, and practices that are common among the client populations that are likely to be served, d) comfort in asking and talking about spiritual issues with clients, and e) a willingness to seek information from appropriate professionals and coordinate care concerning client's spiritual traditions.

Programs of this type have been successfully implemented in the health care field and have proven effective in increasing understanding and sensitivity to a patient's belief system among physicians (Bazan \& Dwyer, 1998). For instance, RISEN (Re-investing Spirituality and Ethics in Networks) has been an ongoing program since 1985 that includes a three-part curriculum of mentor training, an intensive orientation and practicum in personal spirituality, and a seminar in relational ethics. Implementing these organizational trainings can assist clinicians in developing practices and protocols to aid clients in effectively utilize their existing religious coping mechanisms when difficult life circumstances arise (Delaney, Forcehimes, Campbell, \& Smith, 2009). 
Attempts at the policy level to increase cultural sensitivity of health care services are evidenced by the U.S. Department of Health and Human Services, Office of Minority Health recommendations known as the Culturally and Linguistically Appropriate Services (CLAS) Standards. The CLAS is a set of fourteen national standards that were set forth to respond to the need to ensure that all people entering the healthcare system receive equitable and effective treatment in a culturally and linguistically appropriate manner (Hoffman, 2011). These standards were created with the underlying principle that providing culturally appropriate services to individuals has the potential to improve access to care, quality of care, and, ultimately, health outcomes (USDHHS, 2001). As part of providing culturally competent care, National CLAS standards recommend: a) encouraging patients/consumers to express their spiritual beliefs and cultural practices, b) being familiar with and respectful of various traditional healing systems and beliefs and c) integrating these approaches into treatment plans when appropriate.

Social workers providing service delivery to Latino immigrants are in a prime position to advocate for the application of these CLAS standards. When working with recent Latino immigrants, issues of cultural competency can be particularly important. The ability of social workers to put CLAS standards into effect by offering culturally sensitive services that take into account cultural values and beliefs can likely have a beneficial impact on the quality of services provided to recent Latino immigrants.

\section{Future Directions for Research, Intervention, and Policy}

\section{Research}

Previous research on religious coping among Latinos has primarily viewed the construct from a conventional religious perspective. Available standardized scales used 
in measuring religious coping have been constructed from a Judeo-Christian perspective. Yet, along with the surge of Latino immigration has come an increased presence of folk and traditional healing practices in the U.S. (Viladrich \& Abraido-Lanza, 2009). Among Latinos in the U.S., traditional and folk beliefs and the use of traditional healers is widespread (Gallant, Spitze, \& Grove, 2010; Villa-Caballero et al., 2010). In addition to its popularity in the U.S. (Miami, Chicago, New York, Los Angeles, and San Francisco), it has a strong presence in the Caribbean (Puerto Rico and Dominican Republic) as well as many other Latin American countries (McNeill \& Cervantes, 2008). Gloria and Peregoy (1996) report that the three main traditional folk healing systems among Latinos living in the U.S. are: a) Curanderismo, b) Espiritismo, and c) Santería. GonzalezWhippler (2001) estimates over a hundred million practitioners of Santería alone in Latin America and the United States.

Within these belief systems, an individual's life is viewed as a spiritual phenomenon with healing practices based on the premise of mutual interdependence between the physical and spiritual dimensions (Ortiz, Davis, \& McNeil, 2008). These folk healing traditions usually include an amalgamation of sacred rituals, prayers, incantations, and medicinal herbs that are used a means of resolving a host of physical, psychological, and social issues.

Given the prevalence of these traditional belief systems and practices among Latinos, research into the function of such systems as a religious coping mechanism remain relatively scarce. As such, there is considerable need for research in the everexpanding use of folk healing practices among Latinos. Future research directions should include whether these practices serve as an effective coping strategy among Latinos. 
Additionally, investigations are needed to examine associations between folk healing practices and health promoting/compromising behaviors among Latinos. In order to quantitatively assess these practices, there is a demand for the development and psychometric testing of scales measuring the scope of such traditions. Knowledge gained from this research can lead to greater insight as to the function and effect of traditional belief systems in the lives of Latinos in the U.S.

Further epidemiological studies are needed in order to better understand the proximate and distal risk factors of substance abuse and HIV risk behaviors of Latinos. The unanticipated findings in the present study are indicative of the need for research on the influence of religious resources on health behaviors among Latinos. For example, future investigations should include further examination as to the function and influence of negative coping styles on substance use patterns of Latinos.

Results from the present study also suggested that religious coping may have protective influence on the frequency of drug use among drug users. Given the small number of drug using participants in this sample, generalizability is limited. Future studies are needed that examine the effects of religious coping among larger clinical samples of Latino immigrants.

There is also an existing demand to better understand the distinct acculturative stressors experienced by Latino immigrants across a variety of receiving communities. There may be unique acculturative stressors across new and well-established receiving communities (Schwartz et al., 2010). As such, these stressors may impact the health behaviors in Latino immigrants differently. Acculturative stress studies with Latino immigrants have predominantly been conducted in areas of the U.S. with high Latino 
immigrant populations such as Texas (Arbona, Olvera, Rodriguez, Hagan, Linares \& Wiesner, 2010), Florida (Schwartz, Zamboanga \& Jarvis, 2007) and California (Finch \& Vega, 2003). There is a notable shortage of research for Latinos in new/ mono-cultural receiving communities. However, recent census data indicate that Latino populations are undoubtedly rapidly growing in traditionally non-Latino states such as North Carolina and Georgia (U.S. Census, 2011). The immigration experience of Latinos into these states may be plagued with increased obstacles and struggles related to the acculturation process. This is predominantly due to the lack of available supports, unavailability of culturally or linguistically congruent services, and discrimination by receiving communities unfamiliar with the Latino population (e.g., Bender et al., 2004; Prado et al., 2008). Given the way in which the context of reception may shape the acculturation process, it is important to examine ways in which the context of reception influences the acculturative stress of recent Latino immigrants. Gaining knowledge in this area could lead to more culturally based intervention work in areas of the U.S. where Latinos are newly immigrating. Creating a safety net of services in these areas could prove to be especially valuable in ameliorating the acculturative stress of recent Latino immigrants in these communities.

On a community level, future research integrating a community-based participatory approach (CBPR) should be explored and implemented. This specific research approach encourages equity and shared decision making between researchers and community members (Bastida, Tseng, McKeever \& Jack, 2010). It can involve partnerships between academic research institutions, community-based organizations, and local community members as equal partners in addressing local needs of recent 
Latino immigrants. Aims of these initiatives should include capacity building, learning about community needs and structure, and exploring potential alternative models, all with the goal of enhancing community-based service delivery to this population (Wallerstein \& Duran, 2006).

Bastida and colleagues (2010) suggest implementing certain ethical considerations when using a CBPR approach with vulnerable populations. In the development of Beyond Sabor, a culturally tailored community intervention aimed at improving health conditions among adult Mexican Americans, the authors discuss six ethical principles that were taken into account: a) respecting and legitimating the voice of community participants, b) maintaining a high level of financial transparency, c) fairness among all involved parties, d) informed and voluntary consent, e) reciprocity, where all constituents both contribute and receive from the process, and f) equal voice and disclosure, where all participants are accorded equal time and acknowledgement regardless of their role in the project and researchers disclosing their own academic interests (Bastida et al., 2010). It is possible that in applying these principles locally within a CBPR approach a further exploration of the particular acculturative stressors experienced by recent Latino immigrants in South Florida can be successfully accomplished. This may prove to be useful in service delivery as knowledge gained from this process can be applied towards intervention and prevention programs with this population.

Additionally, examining how Latino substance use and HIV risk behaviors are associated with community level stress factors such as level of unemployment, proximity to centers of drug trade, and numbers of retail alcohol outlets could prove to be beneficial 
(Alegria et al., 2006). These findings can be utilized to map approaches in service delivery improvement, as well as gaining better insight into community factors that could prevent or promote substance abuse and HIV risk behaviors among Latino immigrants. Along with addressing community level risk factors, protective influences of religious organization within the community can also be identified and engaged. Developing community research partnerships with these organizations can assist in building community ties. These community partnerships can play a valuable role in assessing whether community interventions are well-received, feasible, or even appropriate among recent Latino immigrants (Alegria et al., 2006).

Thus, community level recommendations include a) testing the effectiveness of community-based prevention/intervention approaches that take into account natural supports systems (e.g., religious organizations) and of those that bring evidence-based treatments to non-clinical environments (academic community-partnerships) and b) evaluate and test collaborative research partnerships with Latino immigrant community members that identify new and innovative approaches to substance use and HIV risk programs (Alegria et al., 2006).

\section{Intervention}

Future prevention and intervention efforts with Latinos should include development and empirical testing of interventions incorporating religious coping resources when applicable. Given the prevalent use of this coping style among Latinos, integrating these cultural resources within substance use programs may prove to be particularly effective with this population. 
Foundations for such interventions have been laid within the general U.S. population as spirituality is an established component of several drug treatment and recovery programs such as Alcoholics Anonymous (AA) and Narcotics Anonymous (NA) (e.g., Pardini et al., 2000; Gorsuch 1995; Miller, 1998; Ritt-Olson et al., 2004). Indeed many strategies used in substance abuse treatment incorporate what is essentially spiritual and/or religious material. The themes of these strategies are rooted in the following areas: creating meaning and purpose in life; developing feelings of connectedness with oneself, others, and a higher power; fostering love, compassion and forgiveness; and, defining personal values related to an individual's philosophy of life (Thoresen, 1998; Miller, 1999).

Recent investigations are finding evidence as to the effectiveness of including religious and spiritual components in substance abuse and HIV-risk intervention with Latinos. Amaro and colleagues (2010) examined the use of a spiritual intervention on the substance abuse and HIV risk behaviors of among urban, low-income Latinas $(n=13)$ in a Boston-based residential treatment program. Treatment consisted of Spiritual SelfSchema (3-S), a weekly 8-session, mindfulness-based, manual-guided, individual intervention targeting substance abuse and HIV risk behaviors. The intervention integrates cognitive behavioral strategies with Buddhist principles and clients' religious/spiritual beliefs (Amaro, Magno-Gatmaytan, Melendez, Cortes, Arevalo, \& Margolin, 2010). 3-S has proven efficacious for reducing drug use and HIV risk behaviors among mixed-gender, methadone-maintained outpatients (Avants, Beitel, \& Margolin, 2005; Margolin et al., 2006). Personal interviews indicated high rates of acceptability of this treatment modality among Latinas. Based on data collected at 
baseline, 2 months and 5 months follow ups, the treatment also appeared to be successful in reducing the number of outcomes relevant to recovery from addiction and to HIV prevention, including impulsivity, spirituality, motivation for change, and HIV prevention knowledge.

Although results from this study are promising further research is needed in order to rigorously assess the efficacy of 3-S therapy among Latinos in substance abuse treatment. Spanish translations of this manualized intervention are easily accessible and available free of cost, making program implementation relatively feasible with other Latino subsamples (Avants, Margolin, Torres-Soto, Cortes, 2003). Future intervention/prevention research should include evaluating the efficacy of 3-S in decreasing the substance abuse and HIV risk behaviors of Latino immigrants.

\section{Policy}

The legal parameters that fall within separation of church and state are a key point of interest when discussing the integration of religious resources within secular service delivery. When incorporating these issues within a secular environment, special attention needs to be given as to what types of religious speech, action, or behaviors are legally and constitutionally acceptable in the workplace (King, 2007).

In order to avert any legal or political issues, an organization needs to become well versed and disseminate information regarding what is acceptable and unacceptable spiritual and/or religious expression within the parameters of the law. This is especially true when it comes to government funded organizations. For example, in 1997, President Bill Clinton issued his "Guidelines of Religious Exercise and Religious Expression in the Federal Workplace." The guiding principle for this release was to legally, attempt to 
establish boundaries as to which religious actions, speech, or other forms of expression were acceptable in the federal workplace. The guidelines suggest that the primary method of determining adequate religious expression is the maintenance of balance between workplace efficiency and employees constitutional rights (King, 2007). According to the court system and the Clinton guidelines, there is a fine line in the decision threshold as to when and how to balance public employee's freedom of speech and religion rights under the First Amendment and the Title VII with their rights to maintain a proper workplace environment.

There is no measure, however, whereby the courts, employers, or others can determine which spiritual behaviors are appropriate and how and when those behaviors violate the First Amendment or Title VII. Because spiritual behaviors are generally not institutionally defined, it is often left to individual perspective. Thus, when planning implementation of a spiritual intervention such as 3-S, it is important for an agency to develop certain limits from the inception as to what is considered acceptable. Creating clear boundaries can be a key component to quelling possible difficult predicaments before they arise (King, 2007).

Given these policy concerns, organizations may be hesitant towards incorporating spiritual and/or religious issues within their prevention and intervention programs. As such, policies like the aforementioned Culturally and Linguistically Appropriate Services (CLAS) Standards should be underscored. Within the context of providing culturally sensitive care, CLAS encourages providers to become familiar and integrate cultural and spiritual beliefs and practices into treatment plans. There is a need for greater integration of these standards within Latino serving organizations. Ensuring that institutions are 
implementing these principles at an optimum capacity can lead to culturally sensitive practices that integrate spiritual and religious cultural values as part of the service delivery process.

The field of social work was among the first to acknowledge the importance of religion and spirituality within a holistic person in the environment perspective (Canda \& Furman, 1999). NASW Standards and Ethics in cultural competency state that social workers should seek to understand and recognize the cultural the strengths of their clients. Social workers should have a knowledge base of their clients' cultures and be able to demonstrate competence in the provision of services that are sensitive to clients' cultures with the inclusion of their religious beliefs (NASW, 2012). In accordance with the ethics of the field, social workers should advocate for their institutions to abide by existing national cultural competence standards. Furthermore, lobbying for federal and local policies with an expanded scope of what is considered culturally appropriate, may facilitate the integration of traditional Latino religious belief and practices into the service delivery process.

\section{Limitations}

The present results should be interpreted in light of several limitations. The investigation utilized secondary data analyses from an ongoing longitudinal investigation examining the influence of pre immigration assets on the health behavior trajectories of recent Latino Immigrants. Information was therefore limited to that which was available in the secondary data set. As such, data regarding the participant's religious demographics including religious affiliation could not be ascertained. Another evident limitation was the use of a sample of convenience. The nature of the sample consisted 
partially of a hidden population - undocumented immigrants. Therefore, the use of respondent driven sampling, a method that has been widely successful in recruiting hidden populations was implemented. Given that this population is difficult to access, this study provided a unique opportunity to examine this understudied group. The investigation also relies on self-report measures of substance use and HIV risk behaviors. In general, the use of self-report measures makes the design susceptible to socially desirable responses. Measures of social desirability were not included in the Recent Latino Immigrant Study. Care was taken, however, to use psychometrically appropriate measures and include experienced Latino interviewers who were extensively trained in culturally appropriate interviewing techniques. Cultural adaptations in research with Latinos, such as employing culturally-informed interviewers and devising well-adapted questionnaires have been found to increase participant satisfaction and provide more accurate data (De La Rosa, Babino, Rosario, \& Valiente, 2011; De La Rosa, Rahill, Rojas, \& Pinto, 2007). The study was also conducted with a fairly young sample (ages 19-42). As such, these findings cannot be generalized to other Latino age groups as generational differences have been found to play a role in frequency and quality of religious coping styles (Koenig, 2006). Lastly, the research design was cross sectional, rather than longitudinal, not allowing for examination of causal inferences and changes in behavior patterns over time. It may be Latino immigrant's religious coping styles change as length of time in the U.S. increases. Future longitudinal studies are needed in order to continue to shed light on the long-term influence of religious coping on the relationship between acculturative stress and substance use/HIV risk behaviors among Latino immigrants. 


\section{Conclusion}

This study aimed to advance the field of social work by contributing to the understanding of how religious coping as a Latino cultural value influences the adaptation process during the immigration experience. Expanding scientific understanding as to the function and effect of these coping mechanisms could lead to enhanced culturally relevant approaches in social service delivery among Latino populations. The primary contributions of this study to the literature appear to be at least threefold. First, the use of an ethnically diverse sample of recent Latino immigrants is an important advance in research with ethnic minority populations. The rise of immigration to the U.S. from a wide array of Latin American countries makes gaining better insight and understanding of this population a critically important social welfare concern. Secondly, the investigation focuses on the largely understudied construct of religious coping among Latinos. Lastly, religion is a central cultural value among Latinos, thus it is important to expand our awareness as to how its use affects the mental and physical well-being of this population. Gaining a better understanding of the stress and coping processes in the lives of recent immigrants can help inform public policy and tailor prevention and intervention programs through targeting the specific needs of this population. Moreover, this knowledge can assist social workers in adequately empowering Latino immigrants in fully becoming healthy members of their host societies (Yakushko et al., 2008). 
Table 1

Framework of Contextual Factors Influencing Acculturation

Prior immigration context

Society of origin factors

Political environment

Economic environment

Social environment

Individual factors

Demographics before immigration

Reason for immigration

Role in the immigration decision

Prior knowledge or contact with host society

Separation from social support networks

Loss of significant others

Immigration context

Type of immigration groups

Route of immigration

Level of danger in the immigration journey

Duration of immigration journey

Settlement context

Society of settlement factors

Political environment

Economic environment

Social environment

Immigration policies

Societal attitudes towards immigration

Adapted from "Measuring acculturation: Where we are and where we need to go" by L. Cabassa, 2003, Hispanic Journal of Behavioral Sciences, 25, p. 131 
Table 2

Dimensions of Acculturative Stress

Dimension Sub-dimensions

Instrumental/Environmental

Financial

Language barriers

Lack of access to health care

Unsafe neighborhoods

Unemployment

Lack of education

Social/Interpersonal

Loss of social networks

Loss of social status

Family conflict

Intergenerational conflicts

Changing gender roles

Societal

Discrimination/stigma

Legal status

Political/historical forces

Adapted from "Latinos, Acculturation, and Acculturative Stress: A Dimensional Concept Analysis" by S. Caplan, 2007, Policy, Politics, \& Nursing Practice, 8, p. 96. Copyright 2008 by Sage Publications. 
Table 3

Inclusion and Exclusion Criteria for Parent Study

\begin{tabular}{|c|c|c|}
\hline Criteria & Inclusion & Exclusion \\
\hline No. of years in the U.S & $\begin{array}{l}\text { Been in the U.S. for one } \\
\text { year or less }\end{array}$ & $\begin{array}{l}\text { Been in the U.S. for more } \\
\text { than one year }\end{array}$ \\
\hline Age & $\begin{array}{l}\text { Between the ages of } 18 \\
\text { and } 34\end{array}$ & $\begin{array}{l}\text { Younger than } 18 \text { and older } \\
\text { than } 34\end{array}$ \\
\hline Country of Birth & $\begin{array}{l}\text { Spanish Speaking Latin } \\
\text { American Countries } \\
\text { (Argentina, Bolivia, } \\
\text { Chile, Colombia, Costa } \\
\text { Rica, Cuba, Dominican } \\
\text { Republic, Ecuador, El } \\
\text { Salvador, Guatemala, } \\
\text { Honduras, Mexico, } \\
\text { Nicaragua, Panama, } \\
\text { Paraguay, Peru, Uruguay, } \\
\text { and Venezuela }\end{array}$ & All Other Countries \\
\hline Gender & Male or Female & $\begin{array}{l}\text { No exclusion based on } \\
\text { gender }\end{array}$ \\
\hline Immigration Status & $\begin{array}{l}\text { Documented or } \\
\text { Undocumented }\end{array}$ & $\begin{array}{l}\text { No exclusion based on } \\
\text { immigrant status }\end{array}$ \\
\hline First time on the U.S. & First Time & Not first time \\
\hline $\begin{array}{l}\text { Willingness to Participate in } \\
\text { two-year study }\end{array}$ & Willing to participate & Not willing to participate \\
\hline $\begin{array}{l}\text { Willingness to Provide } \\
\text { Corroborative Source in the } \\
\text { U.S. }\end{array}$ & Willing to provide & Not willing to provide \\
\hline $\begin{array}{l}\text { Willingness to Provide } \\
\text { Corroborative Source in } \\
\text { Country of Origin }\end{array}$ & Willing to provide & Not willing to provide \\
\hline Residency in the U.S. & $\begin{array}{l}\text { Miami-Dade County, } \\
\text { Florida }\end{array}$ & $\begin{array}{l}\text { All other counties in the } \\
\text { U.S. }\end{array}$ \\
\hline
\end{tabular}


Table 4

Descriptive Characteristics of Sample $(N=415)$

\begin{tabular}{|c|c|c|}
\hline Variables & $\mathrm{n}$ & $\%$ \\
\hline \multicolumn{3}{|l|}{ Gender } \\
\hline Female & 211 & $50.80 \%$ \\
\hline Male & 204 & $49.20 \%$ \\
\hline \multicolumn{3}{|l|}{ Country of Origin } \\
\hline Cuba & 207 & $49.90 \%$ \\
\hline Colombia & 77 & $18.60 \%$ \\
\hline Honduras & 32 & $7.70 \%$ \\
\hline Nicaragua & 31 & $7.50 \%$ \\
\hline Domimican Republic & 5 & $1.20 \%$ \\
\hline Mexico & 7 & $1.70 \%$ \\
\hline Other Central American & 12 & $2.90 \%$ \\
\hline Other South American & 44 & $10.60 \%$ \\
\hline \multicolumn{3}{|l|}{ Education } \\
\hline Less than high school & 42 & $10.10 \%$ \\
\hline High school diploma & 159 & $38.30 \%$ \\
\hline Some traiming/college & 145 & $34.90 \%$ \\
\hline Bachelor's (4-5 years college) & 52 & $12.50 \%$ \\
\hline Graduate/Professional Studies & 17 & $4.10 \%$ \\
\hline \multicolumn{3}{|l|}{ Documentation Status } \\
\hline Documented & 325 & $78.30 \%$ \\
\hline \multirow[t]{2}{*}{ Undocumented } & 90 & $21.70 \%$ \\
\hline & Mean(SD) & Range \\
\hline Age & 28.68 & $19-42$ \\
\hline Armual Income & $7211.71(6082.77)$ & $200-59970$ \\
\hline
\end{tabular}


Table 5

Frequencies for Key Observed Study Variables

\begin{tabular}{|c|c|c|c|c|c|c|c|c|}
\hline \multirow[b]{2}{*}{ Variable } & \multirow[b]{2}{*}{$\mathrm{M}$} & \multirow[b]{2}{*}{ SD } & \multirow[b]{2}{*}{ Skewness } & \multirow[b]{2}{*}{ Kurtosis } & \multicolumn{2}{|c|}{ Actual } & \multicolumn{2}{|c|}{ Possible } \\
\hline & & & & & Min & Max & Min & Max \\
\hline Acculturative Stress & 7.16 & 4.42 & 0.81 & 0.05 & 1 & 20.56 & 0 & 23 \\
\hline Internal Rel. Coping & 45.62 & 18.73 & -0.07 & -1.29 & 15 & 75 & 15 & 75 \\
\hline External Rel. Coping & 17.14 & 10.12 & 1.78 & 2.25 & 10 & 50 & 10 & 50 \\
\hline Positive Rel. Coping & 2.51 & 0.95 & -0.70 & -1.22 & 1 & 4 & 1 & 4 \\
\hline Negative Rel. Coping & 1.42 & 0.52 & 1.74 & 3.97 & 1 & 4 & 1 & 4 \\
\hline Alcohol Quantity ${ }^{\mathrm{a}}$ & 4.93 & 5.07 & 4.06 & 25.52 & 0 & 48 & 0 & $\mathrm{n} / \mathrm{a}$ \\
\hline Alcohol Frequency ${ }^{\text {a }}$ & 8.16 & 12.99 & 2.93 & 11.65 & 0 & 90 & 0 & 90 \\
\hline AUDIT & 4.22 & 4.73 & 1.48 & 2.32 & 0 & 25 & 0 & 40 \\
\hline Drug Use Incidence & 0.07 & 0.26 & 3.25 & 8.59 & 0 & 1 & 0 & 1 \\
\hline Drug Use Frequency & 1.91 & 9.65 & 6.66 & 49.36 & 0 & 90 & 0 & 90 \\
\hline Oral sex condom use & 3.19 & 1.51 & -1.51 & 0.46 & 0 & 4 & 0 & 4 \\
\hline Vaginal sex condom use & 2.05 & 1.84 & -0.07 & -1.85 & 0 & 4 & 0 & 4 \\
\hline Anal sex condom use & 1.90 & 1.94 & 0.10 & -1.95 & 0 & 4 & 0 & 4 \\
\hline
\end{tabular}

Note. ${ }^{\mathrm{a}}$ Non-transformed mean and standard deviation values are presented $;{ }^{\mathrm{b}} 0=$ no drug use in past 90 days, $1=$ drug use at least once in past 90 days. 
Table 6

Correlations of Key Observed Variables

\begin{tabular}{|c|c|c|c|c|c|c|c|c|c|c|c|c|c|c|c|c|}
\hline & Variable & M & SD & 1 & 2 & 3 & 4 & 5 & 6 & 7 & 8 & 9 & 10 & 11 & 12 & 13 \\
\hline 1 & Int. Rel.Coping & 45.62 & 18.73 & 1 & & & & & & & & & & & & \\
\hline 2 & Ext. Rel. Coping & 17.14 & 10.12 & 0.10 & 1 & & & & & & & & & & & \\
\hline 3 & Pos. Rel. Coping & 2.51 & 0.95 & 0.07 & $0.57 * *$ & 1 & & & & & & & & & & \\
\hline 4 & Neg.Rel. Coping & 1.42 & 0.52 & 0.05 & $0.18^{* *}$ & $0.25 * *$ & 1 & & & & & & & & & \\
\hline 5 & Accult. Stress & 7.16 & 4.42 & 0.07 & 0.03 & -0.03 & -0.05 & 1 & & & & & & & & \\
\hline 6 & Alcohol Quant. ${ }^{a}$ & 4.93 & 5.07 & $-0.15^{* *}$ & 0.02 & 0.05 & 0.02 & -0.05 & 1 & & & & & & & \\
\hline 7 & Alcohol Freq. ${ }^{\text {a }}$ & 8.16 & 12.99 & $-0.15^{* *}$ & 0.02 & 0.02 & -0.02 & -0.01 & $0.71^{* *}$ & 1 & & & & & & \\
\hline 8 & Prob. Alcohol Use & 4.22 & 4.73 & $-0.12 *$ & -0.05 & 0.02 & -0.04 & -0.01 & $0.74 * *$ & $0.72 * *$ & 1 & & & & & \\
\hline 9 & Drug Use Incidence ${ }^{\mathrm{b}}$ & 0.07 & 0.26 & -0.02 & $-0.12^{*}$ & -0.06 & 0.00 & 0.01 & 0.02 & 0.00 & 0.01 & 1 & & & & \\
\hline 10 & Drug Use Frequency & 1.91 & 9.65 & -0.04 & -0.1 & -0.02 & $0.12 *$ & -0.09 & $0.14 * *$ & $0.17 * *$ & $0.25 * *$ & $0.70 * *$ & 1 & & & \\
\hline 11 & Oral Sex Cond. Use & 1.34 & 1.53 & -0.04 & 0.1 & 0.07 & -0.02 & -0.01 & -0.02 & -0.05 & 0.02 & 0.04 & 0.10 & 1 & & \\
\hline 12 & Vag. Sex Cond. Use & 1.05 & 0.46 & -0.03 & 0.0 & -0.09 & 0.07 & 0.05 & $-0.11 *$ & -0.04 & $-0.12 *$ & 0.03 & 0.02 & $-0.36^{* *}$ & 1 & \\
\hline 13 & Anal Sex Cond. Use & 1.90 & 1.94 & $-.16^{*}$ & -0.2 & $-0.16^{*}$ & 0.02 & 0.02 & $0.17 *$ & $0.19^{*}$ & $0.24 * *$ & 0.05 & 0.08 & $0.67 * *$ & $0.78 * *$ & 1 \\
\hline
\end{tabular}

Note. ${ }^{* *}=\mathrm{p}<.01 ;{ }^{\mathrm{a}}$ Non-transformed mean and standard deviation values are presented. ${ }^{\mathrm{b}} 0=$ no drug use in past 90 days, $1=$ drug use at least once in past 90 days. 
Figure 1. Measurement Model for Pre-immigration Alcohol Use

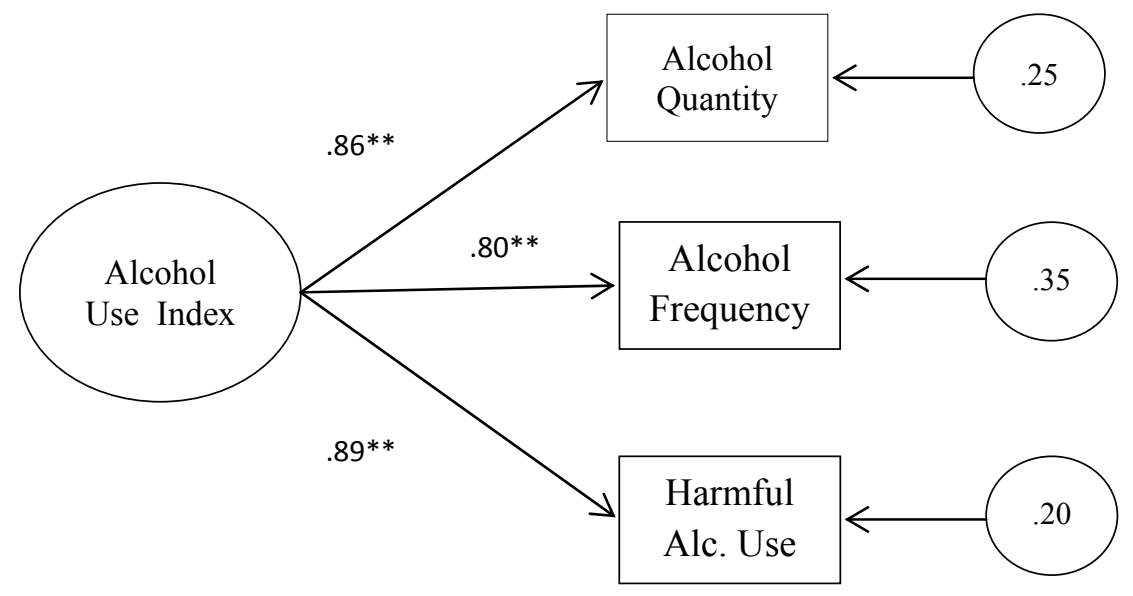

Note. ${ }^{*}=p<.01$. 
Figure 2. Measurement Model for Post Immigration Alcohol Use

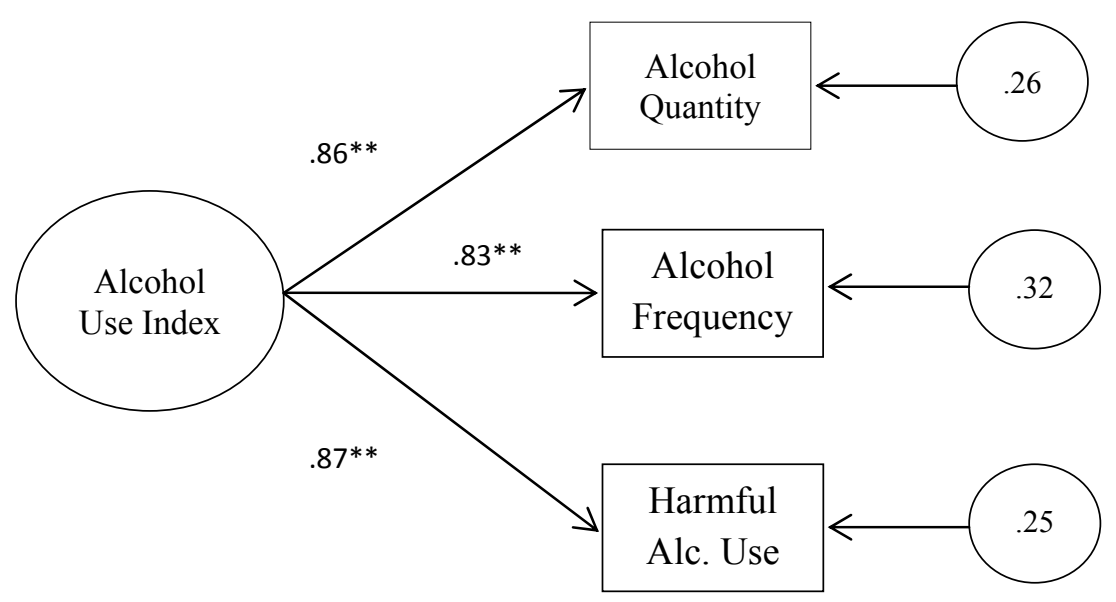

Note. $* *=p<.01$. 
Figure 3. Measurement Model for Pre-Immigration HIV Risk

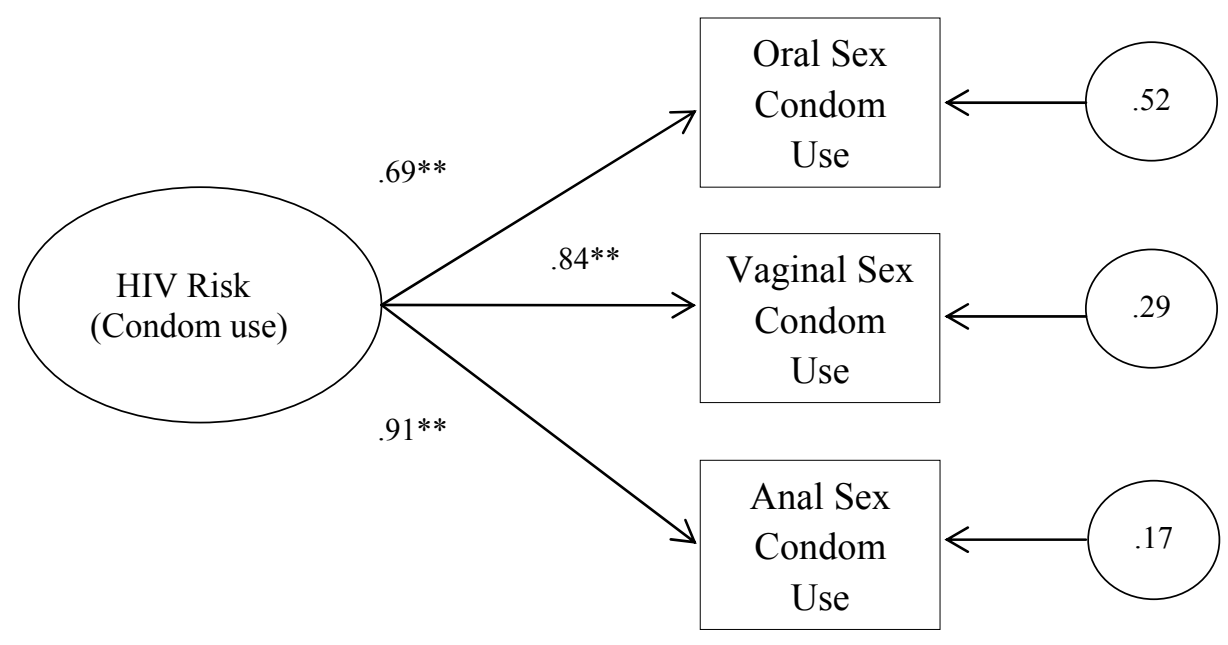

Note. ${ }^{* *}=p<.01$. 
Figure 4. Measurement Model for Post Immigration HIV Risk

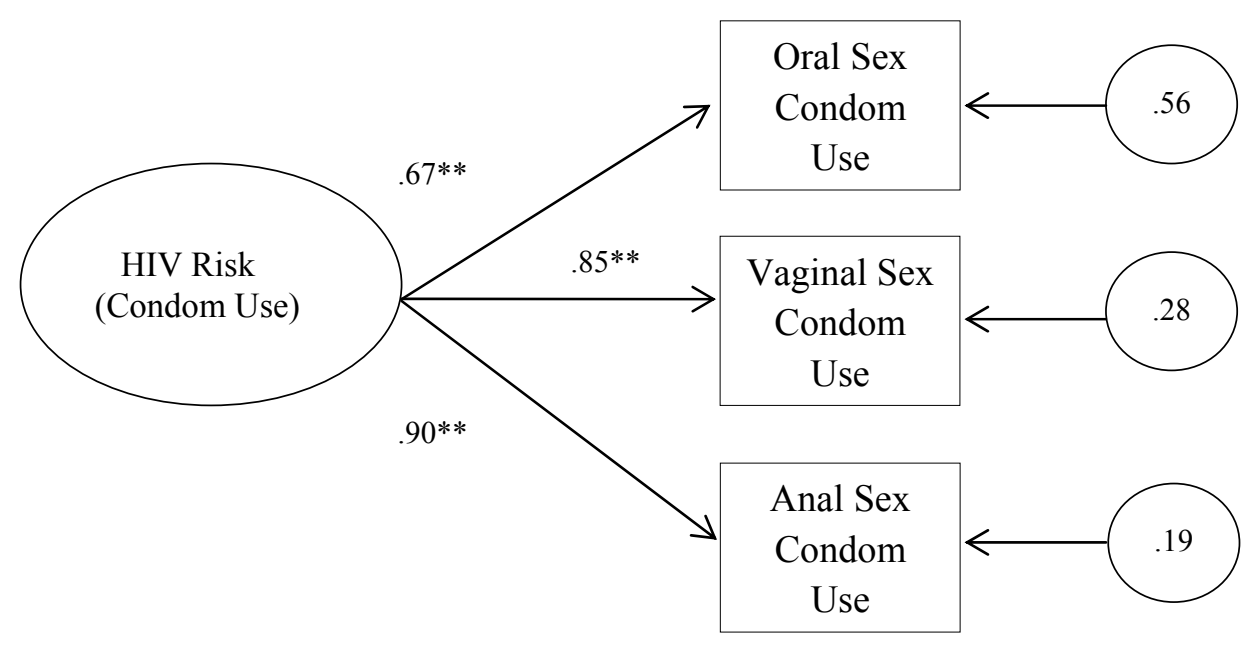

Note. $* *=p<.01$. 
Figure 5. Structural Model Testing Alcohol Use and HIV Risk Outcomes

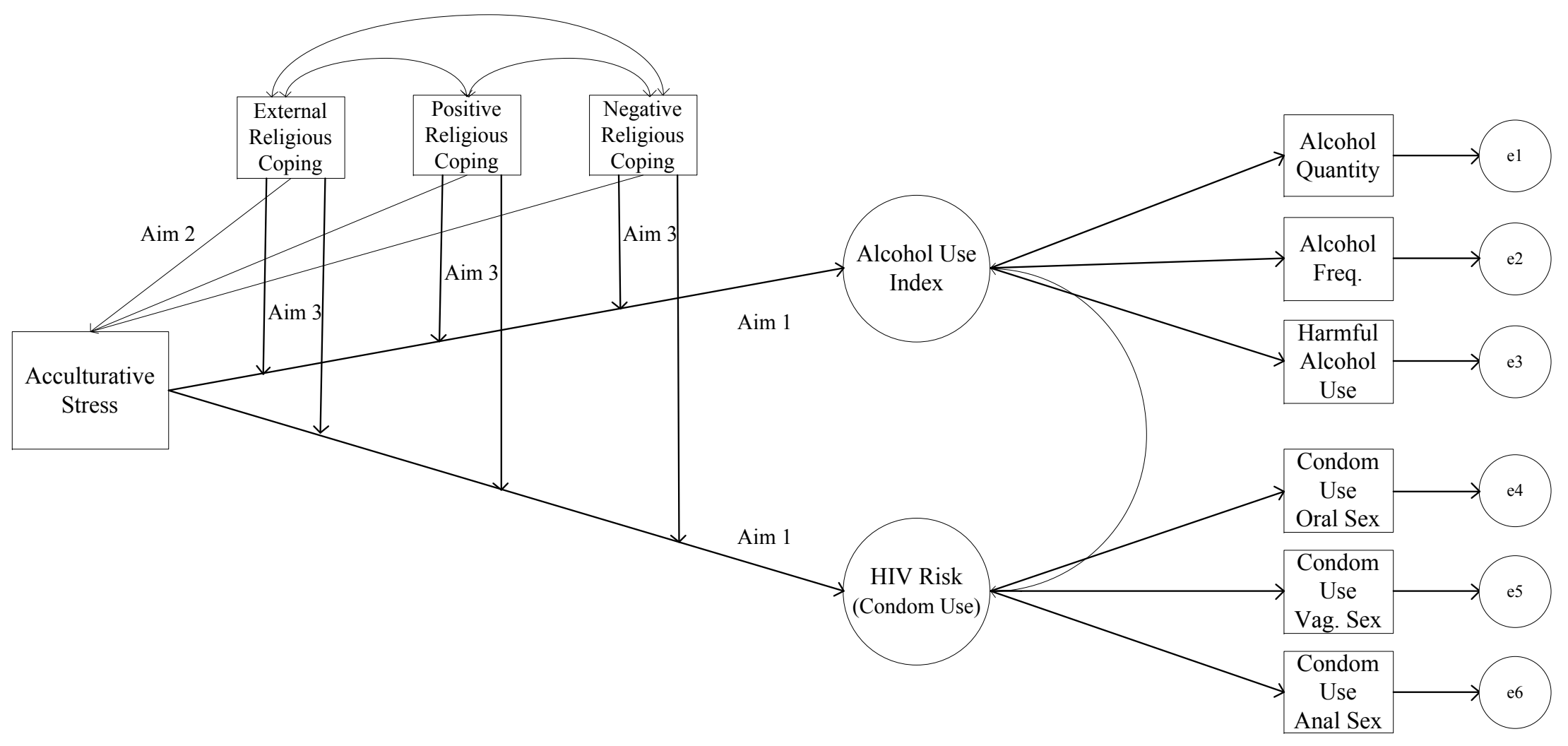


Figure 6. Zero-Inflated Poisson Model Testing Drug Use Outcomes.

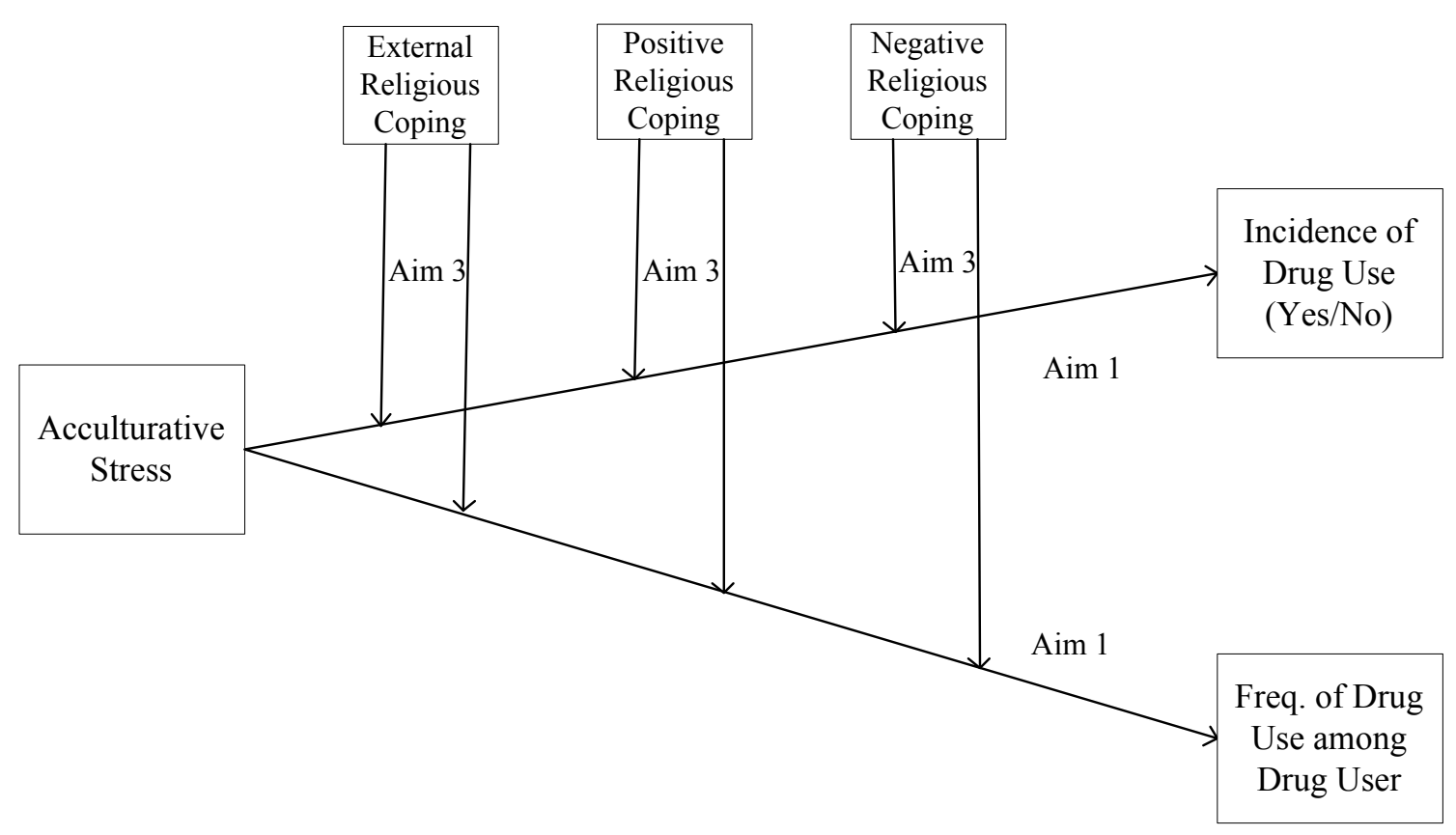


Figure 7. Results of Structural Model Testing Alcohol Use and HIV Risk Outcomes

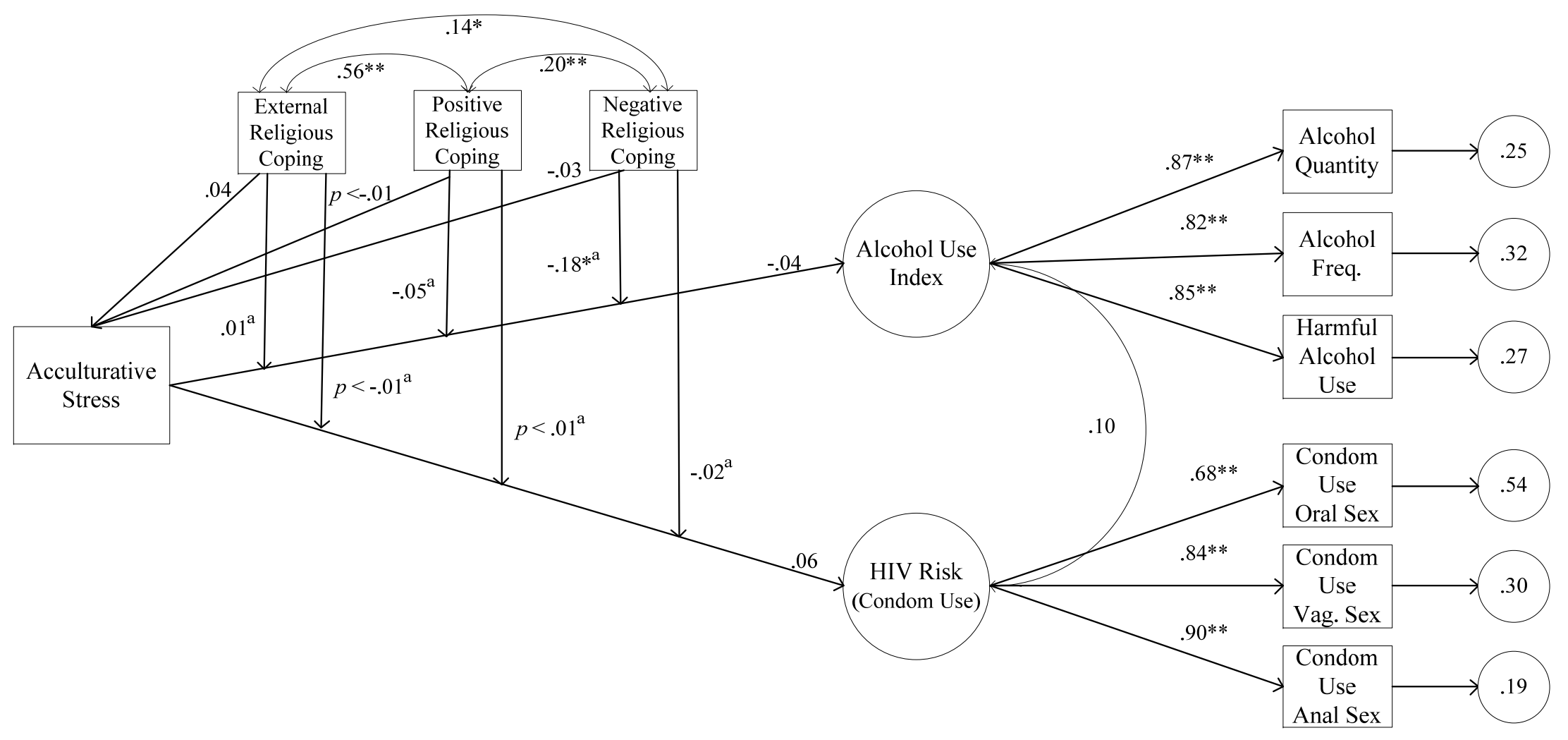

Note. ${ }^{\text {a }}$ Parameters reported in unstandarized path coefficients; $*=p<.05 ; * *=p<.01$. 
Figure 8. Results for Zero-Inflated Poisson Model.

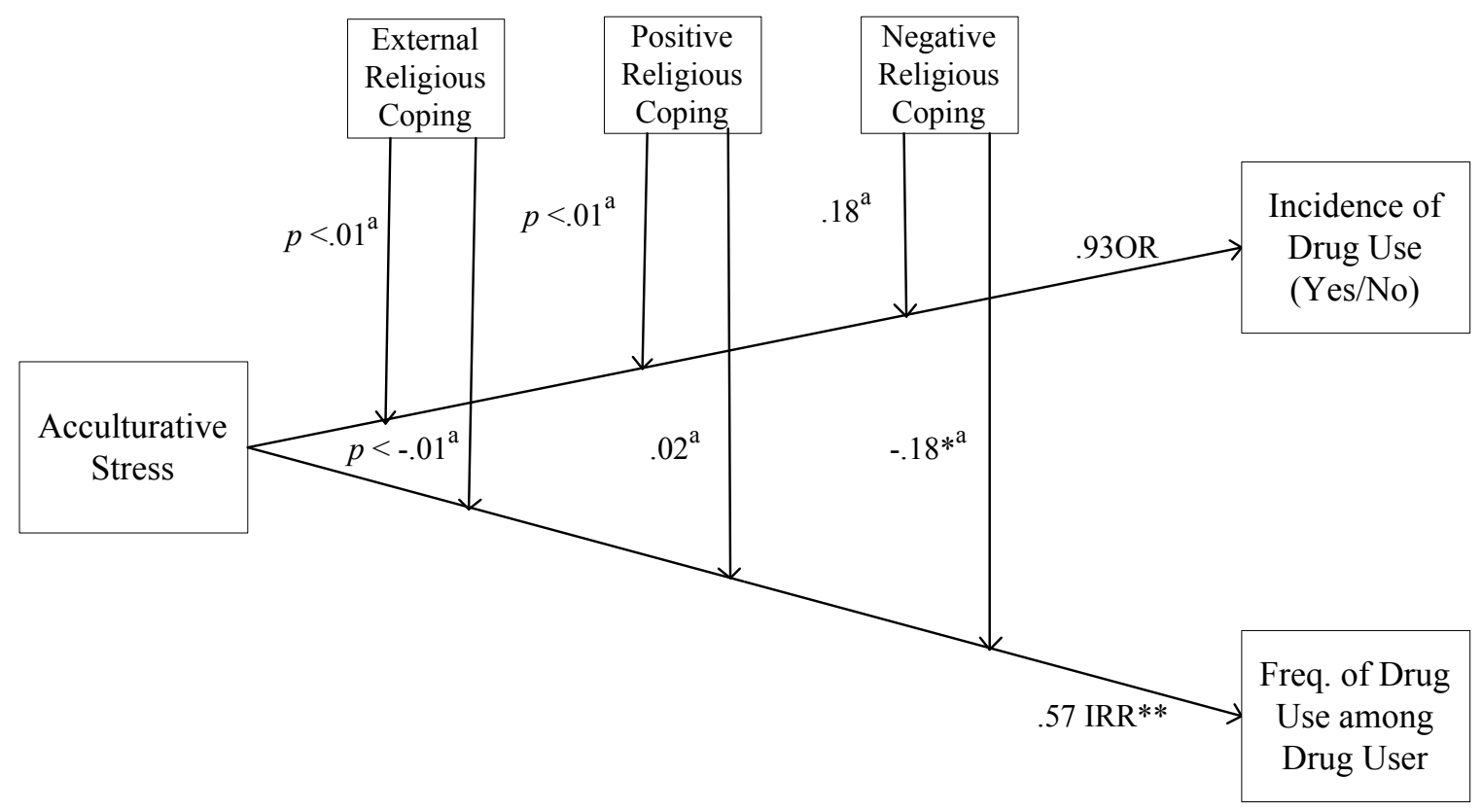

Note. ${ }^{\mathrm{a}}$ Parameters reported in unstandarized path coefficients; $*=p<.05 ; * *=p<.01$; $\mathrm{OR}=$ Odds ratio; $\mathrm{IRR}=$ Indicence rate ratio . 
Figure 9. Moderation Effect by Negative Religious Coping on Relationship between Acculturative Stress and Alcohol Use

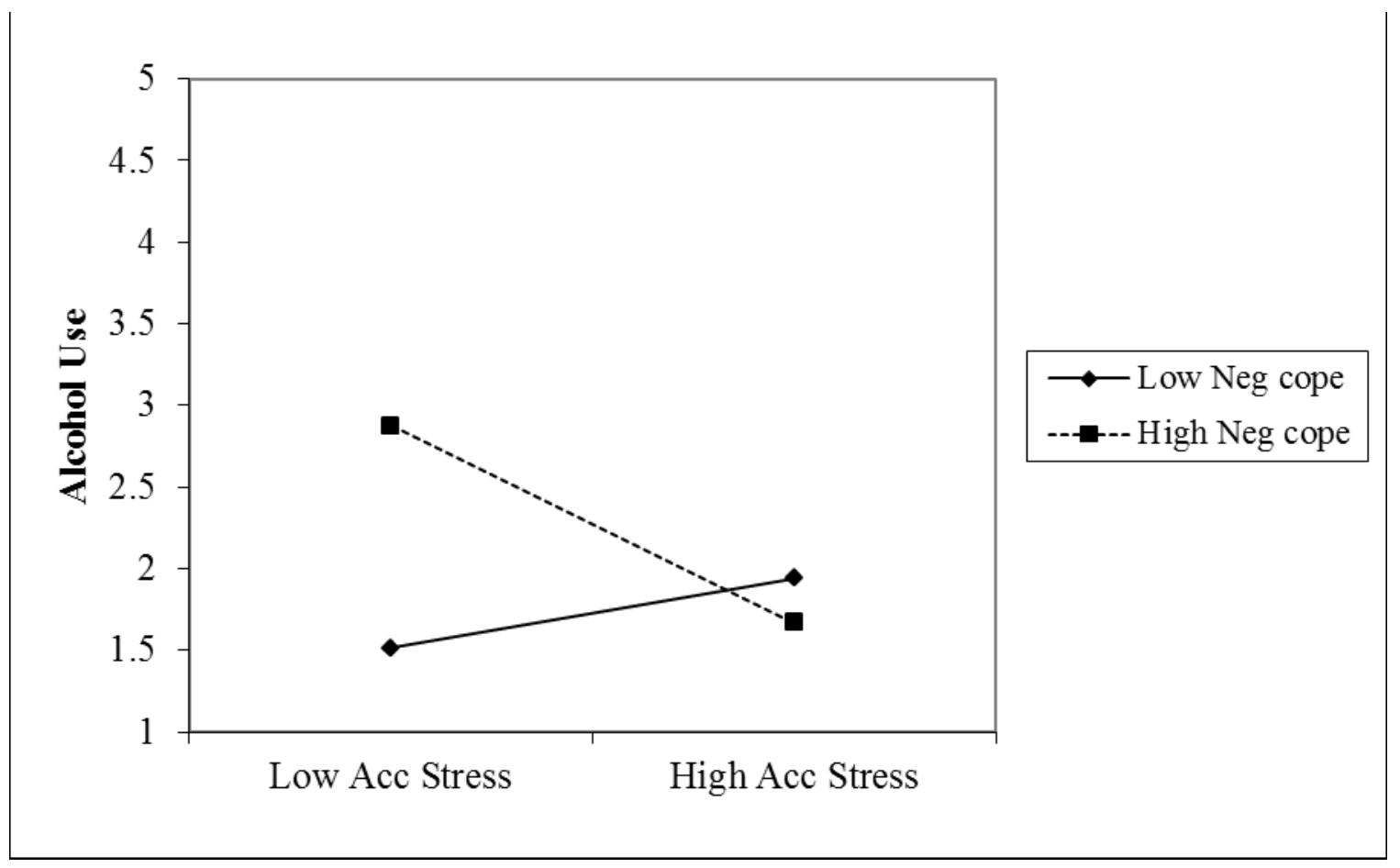


Figure 10. Moderation Effect by Negative Religious Coping on Relationship between Acculturative Stress and Drug Use Frequency

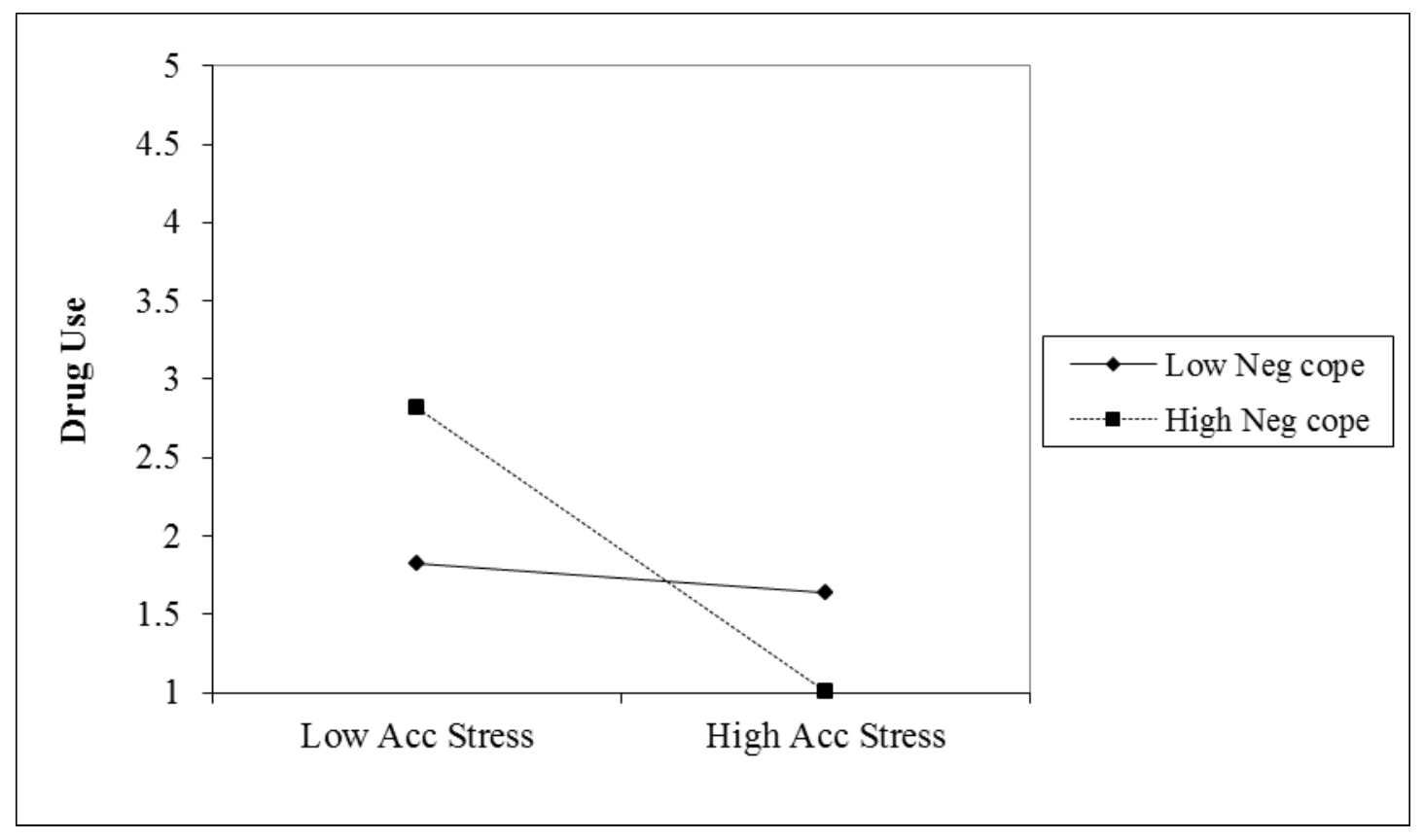


Figure 11. Supplemental Analysis Testing Direct Effects of Religious Coping Mechanisms on Alcohol Use and HIV Risk Behaviors

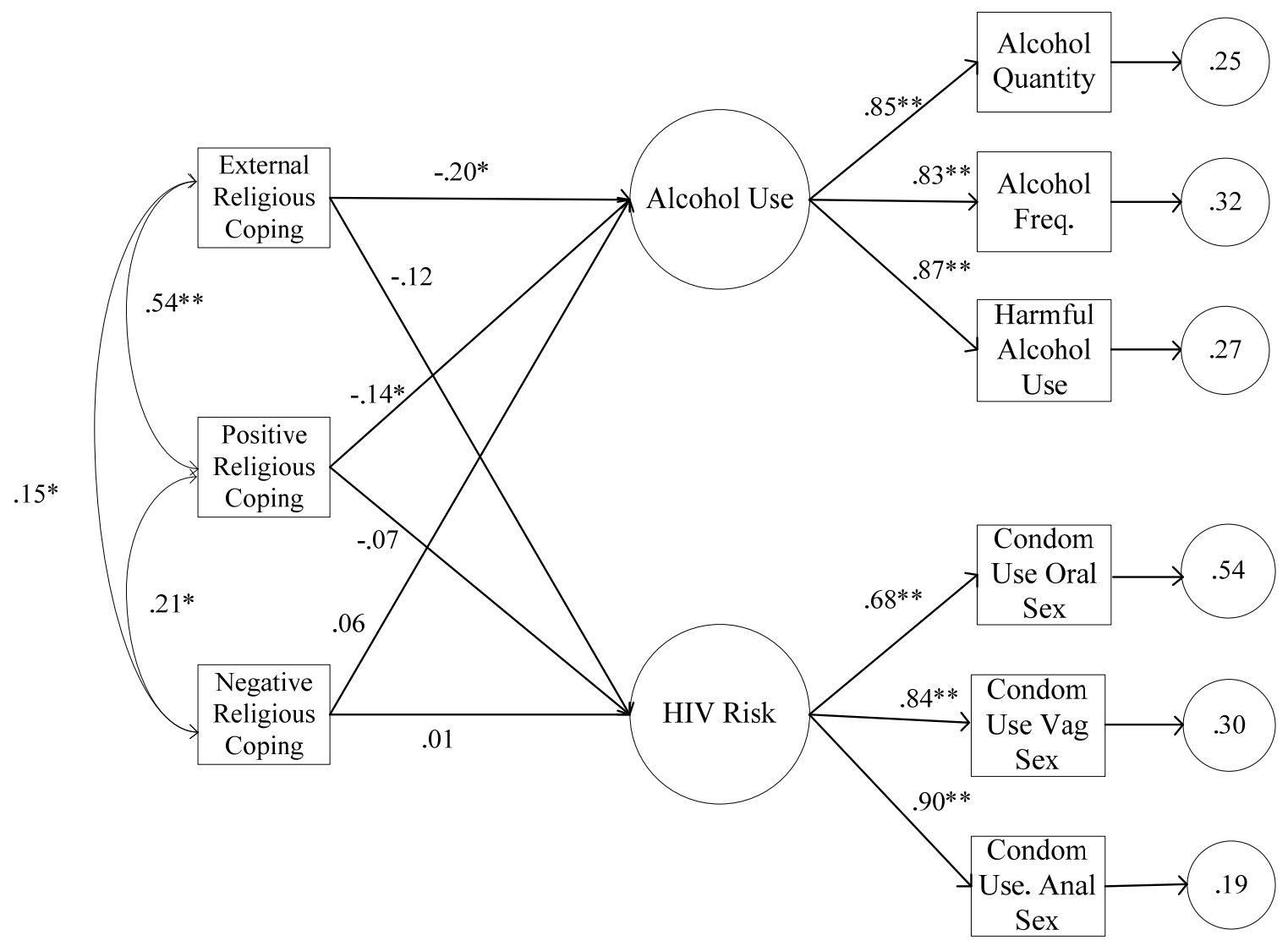

Note. $*=p<.05 ; * *=p<.01$. 
Figure 12. Supplemental Analysis Testing Direct Effects of Religious Coping Mechanisms on Drug Use

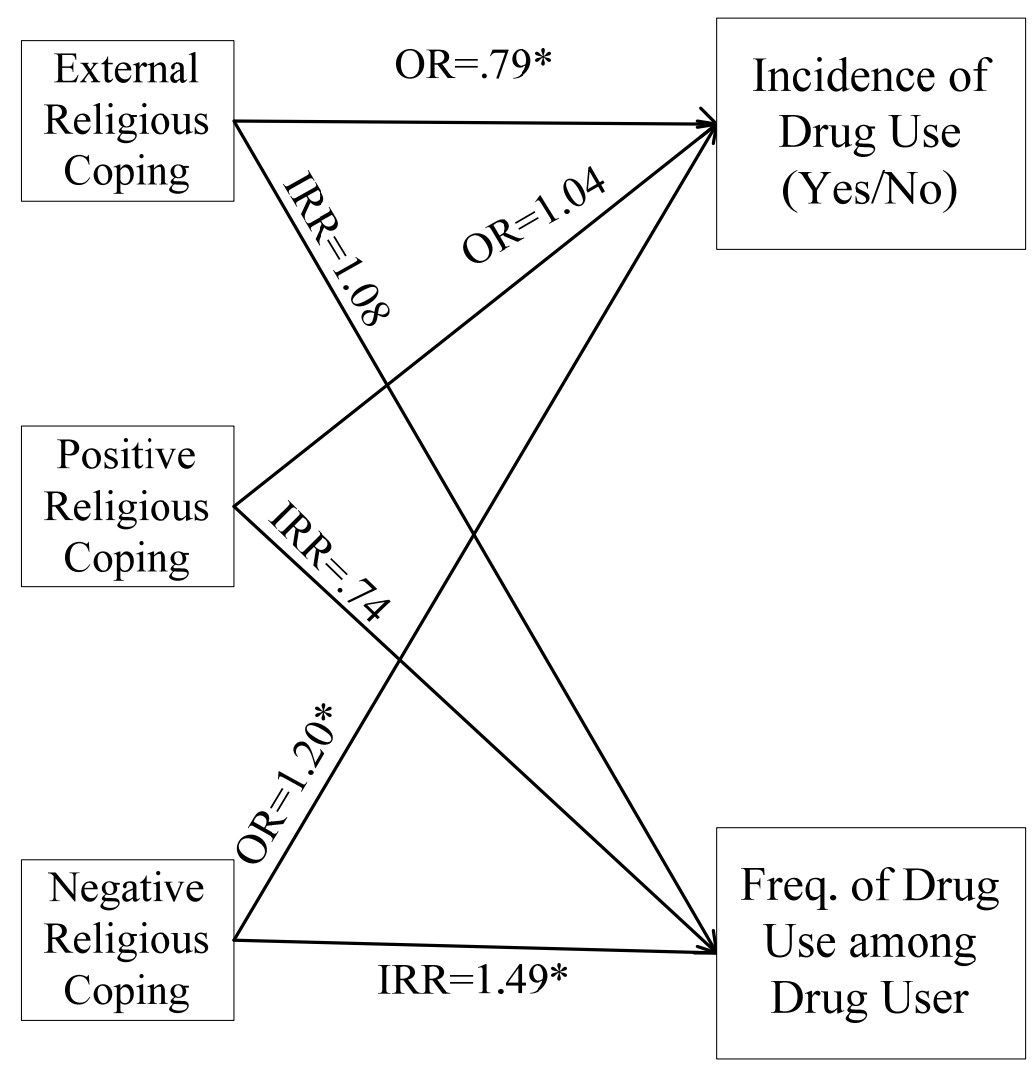

Note. $*=p<.05 ; * *=p<.01 ; \mathrm{OR}=$ odds ratio; $\mathrm{IRR}=$ incidence rate ratio. 


\section{LIST OF REFERENCES}

Abernethy, A.D., Chang, H.T., Seidlitz, L., Evinger, J.S., \& Duberstein, P.R. (2002). Religious coping and depression among spouses of people with lung cancer. Psychosomatics, 43, 456-463. doi: 10.1002/jclp.20049

Abraido-Lanza, A.F., Vasquez, E., \& Echeverria, S.E. (2004). En las Manos de Dios [in God's Hands]: Religious and other forms of coping among Latinos with arthritis. Journal of Consulting and Clinical Psychology, 72, 91-102. doi: 10.1037/0022006X.72.1.91

Abraido-Lanza, A.F., Viladrich, A., Florez, K.R., Cespedes, A., Aguirre, A.N., De La Cruz, A.A. (2007). Fatalismo reconsidered: A cautionary note for health-related research and practice with Latino populations. Ethnicity \& Disease, 17, 153-58 administration. Public Administration Review, Jan./Feb., 103-113.adolescents.

Aiken, L. S., \& West, S. G. (1991). Multiple regression: Testing and interpreting interactions. Newbury Park, CA: Sage.

Alegria, M., Canino, G., Shrout, P. E., Woo, M., Duan, N., Vila, D., . .Meng, X.-L. (2008). Prevalence of mental illness in immigrant and non-immigrant U.S. Latino groups. American Journal of Psychiatry, 165, 359-369.

Alegria, M., Page, J.B., Hansen, H., Cauce, A.M., Obles, R., Blanco, C...,Berry, P. (2006). Improving drug treatment services for Hispanics: Research gaps and scientific opportunities. Drug and Dependence, $84 S$, S76-S84. doi:10.1016/j.drugalcdep.2006.05.009

Alferi, S. M., Culver, J. L., Carver, C. S., Arena, P. L., \& Antoni, M. H. (1999). Religiosity, religious coping, and distress: A prospective study of Catholic and Evangelical Hispanic women in treatment for early-stage breast cancer. Journal of Health Psychology, 4, 1359-1053.

Allen, J.P., Litten, R.Z., Fertig, J.B., Babor, T. (1997). A review of research on the Alcohol Use Disorders Identification Test (AUDIT). Alcoholism: Clinical and Experimental Research, 21, 613-619.

Amaro, H., Magno-Gatmaytan, C., Melendez, M., Cortes, D.E., Arevalo, S. \& Margolin, A. (2010). Addiction treatment intervention: An uncontrolled prospective pilot study of Spiritual Self-Schema Therapy with Latina Women. Substance Abuse, 31, 117-125. doi: 10.1080/08897071003641602

Amaro, H., Vega, R.R., Valencia, D. (2001). Gender, context and HIV prevention among Latinos. In: Aguirre-Molina M, Molina C, Zambrana RE, eds. Health Issues in the Latino Community (pp. 301-324). San Francisco, Ca: Jossey-Bass; 2001:301-324. 
Amaro, H., Whitaker, R., Coffman, G., \& Heeren, T. (1990). Acculturation and marijuana and cocaine use: Findings from HHANES 1982-84. American Journal of Public Health, 80(Suppl.), 54-60.

Anderson, J.E., Mosher, W.D., Chandra, A. (2006). Measuring HIV risk in the U.S. population aged 15-44: Results from Cycle 6 (2002) of the National Survey of Family Growth. Advance data from vital and health statistic. Hyattsville, MD: National Center for Health Statistics.

Ano, G.G., \& Vasconcelles, E.B. (2005). Religious coping and psychological adjustment to stress: a meta-analysis. Journal of Clinical Psychology, 61, 461480 .

Antshel, K.M. (2002). Integrating culture as a means of improving treatment adherence in the Latino population. Psychology, Health \& Medicine, 7, 435-449.

Arbona, C., Olvera, N., Rodriguez, N., Hagan, J., Linares, A., \& Wiesner, M. (2010). Acculturative stress among documented and undocumented Latino immigrants in the United States. Hispanic Journal of Behavioral Sciences, 32, 362-384.

Arnada, M. (2008). Relationship between religious involvement and psychological wellbeing: A social justice perspective. Health \& Social Work, 33, 9-21.

Atkins, D.C., \& Gallop, R.J. (2007). Rethinking how family researchers model infrequent outcomes: A tutorial on count regression and zero-inflated models. Journal of Family Psychology, 21, 726-735.

Avants, S. K., Marcotte, D., Arnold, R., \& Margolin, A. (2003). Spiritual beliefs, world assumptions, and HIV risk behavior among heroin and cocaine users. Psychology of Addictive Behaviors, 17, 159-162.

Avants, S.K., Beitel, M., \& Margolin, A. (2005). Making the shift from 'addict self' to 'spiritual self': Results from a Stage I study of Spiritual Self-Schema (3-S) therapy for the treatment of addiction and HIV risk behavior. Mental Health, Religion, \& Culture, 8, 167-177.

Babor, T. F., Biddle-Higgins, J. C., Saunders, J. B., \& Monteiro, M. G. (2001). The alcohol use disorders identification test: Guidelines for use in primary health care. Geneva,Switzerland: World Health Organization.

Baez, A. \& Hernandez, D. (2001). Complementary spiritual beliefs in the Latino community: The interface with psychotherapy. American Journal of Orthopsychiatry, 71, 408-415.

Bastida, E.M., Tseng, T., McKeever, C., \& Jack, L. (2010). Ethics and community based participatory research: Perspectives from the field. Health Promotion Practice, 11, 16-20. 
Bauer, D.J., \& Curran, P.J. (2011). Introduction to Structural Equation Modeling: Course Notes.

Bazan, W.J. \& Dwyer, D. (1998). Assessing spirituality. Health Progress, 79. Retrieved March 11, 2009, from Catholic Health Association of the United States Web site via http://www.chausa.org/Pub/MainNav/News/HP/Archive/1998/03MarApr/Articles Features/hp9803d tm

Bazargan, S., Sherkat, D., \& Bazargan, M. (2004). Religion and alcohol use among African-American and Hispanic inner-city emergency care patients. Journal for the Scientific Study of Religion, 43, 419-428.

Behling, O. \& Law, K.S. (2000). Translating Questionnaires and Other Research Instruments: Problems and Solutions. Thousand Oaks: Sage Publications.

Bender, D.E., Clawson, M., Harlan, C., \& Lopez, R. (2004). Improving access for Latino immigrants: Evaluation of language training adapted to the needs of health professionals. Journal of Immigrant Health, 6(4), 197-209.

Benet-Martinez, V. (2003). The Riverside Acculturation Stress Inventory (RASI): Development and psychometric properties. Technical Report, Department of Psychology, University of California at Riverside.

Bentler, P.M. \& Chou, C.P. (1987). Practical issues in structural modeling. Sociological Methods and Research, 16, 78-117.

Berry , J. W. Kim, U. (1988). Acculturation and mental health. In P. Dasen J. W. Berry N. Sartorius (Eds.), Health and Cross Cultural Psychology (pp. 207-236). London: Sage.

Berry, J.W. (1997). Immigration, acculturation, and adaptation. Applied Psychology, 46, 5- 68. doi: 10.1080/026999497378467

Berry, J.W., \& Sam, D. (1997). Acculturation and adaptation. In Berry, J.W., Segall, M.H., \& Kagitcibasi, C. (Eds.) Handbook of Cross-Cultural Psychology. Vol.3, Social Behavior and Applications (pp.291-326). Boston: Allyn \& Bacon.

Berry, J.W., Kim, U., Minde, T., \& Mok, D. (1987). Comparative studies of acculturative stress. International Migration Review, 21, 491-511.

Bethel, J.W., \& Schenker, M.B. (2005). Acculturation and smoking patterns among Hispanics: A review. American Journal of Preventive Medicine, 29, 143-148.

Billings, A. \& Moos, R.H. (1982). Stressful life events and symptoms: A longitudinal model. Health Psychology, 1, 99-117. doi: 10.1037/0278-6133.1.2.99 
Billy, J., Tanfer, K., Grady, W.R., \& Klepinger, D.H. (1993) The sexual behavior of men in the United States. Family Planning Perspectives, 25, 52-60.

Bishop, D. (2007). Adult religious education as transformative learning: The use of religious coping strategies as a response to stress. Dissertation Abstract International Section A: Humanities and Social Sciences 68(6-A), 2278.

Blake, S.M., Ledskv, R., Goodenow, C., \& Donnell, L. (2001). Recency of immigration, substance use, and sexual behavior among Massachusetts adolescents. American Journal of Public Health, 91, 794-798. doi: 10.2105/AJPH.91.5.794

Bollen, K. \& Long, S. (1993). Testing structural equation models. Newbury Park: Sage.

Bollen, K.A. (1989). Structural Equations with Latent Variables. New York: John Wiley \& Sons, Inc.

Borges, G., Medina-Mora, M.E., Lown, A., Ye. Y., Robertson, M.J., Cherpitel, C., Greenfield, T. (2006). Alcohol use disorders in national samples of Mexicans and Mexican-Americans: The Mexican National Addiction Survey and the U.S. National Alcohol Survey. Hispanic Journal of Behavioral Science, 28, 425-449.

Boudreaux, E., Catz, S., Ryan, L., Amaral-Melendez, A., \& Brantley, P.J. (1995). The ways of religious coping scale: Reliability, validity, and scale development. Assessment, 2, 233-244. doi: 10.1177/1073191195002003004

Bowen-Reid, T.L. \& Smalls, C. (2004). Multidimensional assessment of spirituality, stress levels and health promoting behaviors amongst African-American college students. Journal of Black Western Studies, 28, 283-291.

Brown, J.M., Council, C.L., Pene, M.A., \& Gfroerer, J.C. (2005). Immigrants and substance use: Findings from the 1999-2001 national surveys on drug use and health (DHHS publication no. SMA 04-3909, analytic series A-23). Rockville, MD: Department of Health and Human Services, Substance Abuse and Mental Health Services Administration, Office of Applied Studies.

Burdette, A.M., Ellison C.G., Hill, T.D., \& Glenn, N.D. (2009). "Hooking up" at college: Does religion make a difference? Journal of the Scientific Study of Religion, 48, 535-551.

Byrne, B.M. (2001). Structural equation modeling with AMOS: Basic concepts, applications, and programming. Mahwah, NJ: Lawrence Erlbaum Associates.

Cabassa, L.J. (2003). Measuring acculturation: Where we are and where we need to go. Hispanic Journal of Behavioral Sciences, 25, 127-146. doi:

10.1177/0739986303025002001 
Caetano, R. \& Mora, M.E. (1998). Acculturation and drinking among people of Mexican descent in Mexico and the United States. Journal of Studies on Alcohol, 49, 462-71.

Canda, E.R. \& Furman, L.D. (1999). Spiritual diversity in social work practice: The heart of helping. New York: The Free Press.

Canda, E.R., Nakashima, M., Burgess, V.L., Russel, R., \& Barfield S.T. (2003). Spirituality diversity and social work: A comprehensive bibliography with annotations $\left(2^{\text {nd }}\right.$ ed.). Alexandria, VA: Council on Social Work Education.

Caplan, S. (2007) Latinos, acculturation, and acculturative stress: A dimensional concept analysis. Policy, Politics, and Nursing Practice, 8, 93-106. doi: $10.1177 / 1527154407301751$

Castro, F.G., Cota, M.K., \& Vega, S. (1999). Health promotion in Latino populations: A sociocultural model for program planning, development, and evaluation. In Huff, R.M., Kline, M.V. (Eds.) Health in Multicultural Populations: A Handbook for Practitioners. Thousand Oaks: Sage Publications,

Cavazos-Rehg, P. A., Zayas, L. H., \& Spitznagel, E. L. (2007). Legal status, emotional well-being and subjective health status of Latino immigrants. Journal of the National Medical Association, 99, 1126-1131.

Centers for Disease Control and Prevention. (2009). HIV/AIDS Surveillance Report, 2007. Vol. 19.

Centers for Disease Control and Prevention. (2010). Florida 2010 Profile.

Centers for Disease Control and Prevention. (2011). HIV among Latinos.

Cervantes, R.C., Padilla, A.M., \& Salgado de Snyder, N. (1990). The Hispanic stress inventory: A culturally relevant approach to psychosocial assessment.

Psychological Assessment, 3, 438-447. doi: 10.1037/1040-3590.3.3.438

Chatters, L.M. (2000). Religion and health: Public health research and practice. Annual Review of Public Health, 21, 335-367.

Cherpitel C.J., \& Borges G. A. (2001). Comparison of substance use and injury among Mexican American emergency room patients in the United States and Mexicans in Mexico. Alcoholism: Clinical and Experimental Research, 25,1174-1180. doi: 10.1111/j.1530-0277.2001.tb02332.x

Cherpitel, C.J. (1998). Differences in performance of screening instruments for problem drinking among blacks, whites, and Hispanics in an emergency room population. Journal of Studies on Alcohol, 59, 420-426. 
Cherpitel. C.J. (1997). Comparison of screening instruments for alcohol problems between black and white emergency room patients from two regions of the country. Alcoholism: Clinical and Experimental Research, 21, 1391-1397.

Cheung, G.W., \& Rensvold, R.B. (2002). Evaluating goodness-of-fit indexes for testing measurement invariance. Structural Equation Modeling, 9, 233-255.

Cnann, R.A., Wineburg, R.J., \& Boddie, S.C. (1999). The Newer Deal. New York: Columbia University Press

Cohen, J., Cohen, P., West, S. G., \& Aiken, L. S. (2003). Applied multiple regression/correlation analysis for the behavioral sciences (3rd ed.). Mahwah, NJ: Erlbaum.

Coon, D.W., Rupert, M., Solano, N., Mausbach, B., Kraemar, H., Argulles, T., ...Gallagher-Thompson, D. (2004). Well-being, appraisal, and coping in Latina and Caucasian female dementia caregivers: Findings from the REACH study. Aging and Mental Health, 8, 330-345. doi: 10.1080/13607860410001709683

Cortés, D. E. (1994). Acculturation and its relevance to mental health. In R. G. Malgady \& O. Rodriguez (Eds.), Theoretical and conceptual issues in Hispanic mental health (pp. 54-67). Malabar, FL: Kreiger.

Crane, K.R. (2003). Faith, Family, and Ethnicity in the Second Generation. New York: LFB Scholarly Publishing.

Cuéllar, I., Arnold, B., \& Maldonado, R. (1995). Acculturation Rating Scale for Mexican Americans-II: A revision of the original ARSMA scale. Hispanic Journal of Behavioral Sciences, 17, 275-304.

Davila, Y.R. (2000). Hispanic women and AIDS: Gendered risk factors and clinical implications. Issues in Mental Health Nursing, 21, 635-646.

Dawson, J. F., \& Richter, A. W. (2006). Probing three way interactions in moderated multiple regression: Development and application of a slope difference test. Journal of Applied Psychology, 91, 917-926.

De Frias, C.M. \& Dixon, R.A. (2005). Confirmatory factor structure and measurement invariance of the memory compensation questionnaire. Psychological Assessment, 17, 168-178.

De La Rosa, M., Babino, R., Rosario, A.,Valiente, N., Aijaz, N. (2012). Challenges and Strategies in Recruiting, Interviewing, \& Retaining Recent Latino Immigrants in substance abuse and HIV Epidemiologic Studies. American Journal on Addictions, 21, 11-22. doi: 10.1111/j.1521-0391.2011.00193.x 
De La Rosa, M., Rahill, G. J., Rojas, P., \& Pinto, E. (2007). Cultural adaptations in data collections: Field experiences. Journal of Ethnicity in Substance Abuse, 6, 163180.

De La Rosa, M., Sanchez, M., Dillon, F.R., Ruffin, B.R., Blackson, T., \& Schwartz, S. (in press). Alcohol Use among Latinos: A comparison of pre-immigration, post immigration, and U.S. Born Latinos. Journal of Immigrant and Minority Health.

Delaney, H.D., Forcehimes, A.A., Campbell, W.P., Smith, B.W. (2009). Integrating spirituality into alcohol treatment. Journal of Clinical Psychology, 65, 185-198.

Delgado, M., Lundgren, L.M., Deshpande, A., Lonsdale, J., \& Purington, T. (2008). The association between acculturation and needle sharing among Puerto Rican injection drug users. Evaluation and Program Planning, 3, 83-91.

Desrosiers, A. \& Miller, L. (2008). Substance use versus anxiety in adolescents: Are some disorders more spiritual than others? Research in the Social Scientific Study of Religion, 19, 237-253.

Dillon, F.R., Turner, C.W., Robbins, M.S., \& Szapocznik, J. (2005). The concordance among biological, interview, and self-reported measures of drug use among African American and Hispanic adolescents referred for drug abuse treatment. Psychology of Addictive Behaviors, 19, 404-413.

Dolman, J.M. \& Hawkes, N.D. (2005). Combining the audit questionnaire and biochemical markers to assess alcohol use and risk of alcohol withdrawal in medical inpatients. Alcohol \& Alcoholism, 40, 515-519.

doi:10.1093/alcalc/agh189

Dunn, M., \& O'Brien, K. (2009). Psychological health and meaning in life. Hispanic Journal of Behavioral Sciences, 31, 204-227.

Edwards, L.M., Fehring, R.J., Jarrett, K.M., Haglund, K.A. (2008). The influence of religiosity, gender, and language preference acculturation on sexual activity among Latino/a adolescents. Hispanic Journal of Behavioral Sciences, 30, $447-$ 462. doi: $10.1177 / 0739986308322912$

Elifson, K.W., Klein, H., \& Sterk, C.E. (2003). Religiosity and HIV risk behavior among at risk women. Journal of Religion and Health, 42, 47-66. doi: 10.1023/A:1022264711670

Ellison C.G. \& Levin, J.S. (1998). The religion-health connection: Evidence, theory and future directions. Health Education \& Behavior, 25, 700-720.

Ellison, C.G., Finch, B.F., Ryan, D.N., \& Salinas, J.J. (2009). Religious involvement and depressive symptoms among Mexican-origin adults in California. Journal of Community Psychology, 37, 171-193. 
Ennis, S R., Ríos-Vargas, M. and Albert, N.G. (2011). The Hispanic Population: 2010. C2010BR-04. Washington, D.C.: U.S. Census Bureau, May. http://www.census.gov/prod/cen2010/briefs/c2010br-04.pdf

Epstein, J.A., Botvin, G.J., Dusenbery, L., Diaz, T., \& Kerner, J. (1996). Validation of an acculturation measure for Hispanic adolescents. Psychological Reports, 79, $1075-1079$.

Escobar, J. I.,\& Vega, W. A. (2000). Mental Health and Immigration "AAA's": Where are we and where do we go from here? Journal of Nervous and Mental Disease, $188,736-740$.

Espinosa, G., Virgilio, E., \& Miranda, J. (2003). Hispanic churches in American public life: Summary of findings. Interim Reports, 2, 1-28.

Fierros-Gonzalez, R., Brown, J.M. (2002). High risk behaviors in a sample of MexicanAmerican college students. Psychological Reports, 90, 117-130.

Finch B.K., Kolody, B., \& Vega, W.A (2000). Perceived discrimination and depression among Mexican origin adults in California. Journal of Health \& Social Behavior, 41, 295-313.

Finch, B.F. \& Vega W.A. (2003). Acculturation stress, social support and self-rated health among Latinos in California. Journal of Immigrant Health, 5, 109-117.

Fisher, D. G., Reynolds, G. L., Moreno-Branson, C. M., Jaffe, A., Wood, M. M., Klahn, J. A., \& Muniz, J. F. (2004). Drug treatment needs of Hispanic drug users in Long Beach, CA. Journal of Drug Issues, 34, 879-894.

Fitchett, G., Murphy, P., Kim, J., Gibbons, L., Cameron, J.R, \& Davis, J. (2004). Religious struggle: Prevalence, correlates and mental health risks in diabetic, congestive heart failure, and oncology patients, International Journal of Psychiatry in Medicine, 34, 179-196.

Fitchett, G., Murphy, P.E., Kravitz, H.M., Everson-Rose, S.A., Krause, N.M. \& Powell, L.H. (2007). Racial/ethnic differences in religious involvement in a multi-ethnic cohort of midlife women. Journal for the Scientific Study of Religion, 46, 119132.

Flaskerud, J. H., \& Uman, G. (1993). Directions for AIDS education for Hispanic women based on analyses of survey findings. Public Health Reports, 108, 298304.

Florida Department of Health (2011). Fact Sheet: HIV/AIDS Among Hispanics.

Florida Department of Health. (2009). Fact Sheet: HIV/AIDS US Versus Florida. 
Ford, A. E., \& Norris, K. (1994). Condom beliefs in urban, low income, African American and Hispanic youth. Health Education Quarterly, 21, 39-53.

Ford, K., King, G., Nerenberg, L., \& Rojo, C. (2001). AIDS knowledge and risk behaviors among Midwest migrant farm workers. AIDS Education and Prevention, 13, 551-560.

Fosados, R., Valente, T.W., Caballero-Hoyos, J.R., Torres-Lopez, T. (2005). Condom use and migration to the US: The case of Mexican migrants; Paper presented at the HIV Research: The next generation, Center for HIV Identification, Prevention and Treatment Services (CHIPTS); Los Angeles, CA. 2005; 2005.

Frazier, P.A., Tix, A.P. \& Barron, K.E. (2004). Testing moderator and mediator effects in psychological research. Journal of Counseling Psychology, 15, 115-134. doi: 10.1037/0022-0167.51.1.115

Fry, R. (2008). Latino Settlement in the New Century. Washington, DC: Pew Hispanic Center.

Gallant, M. P., Spitze, G., \& Grove, J. G. (2010). Chronic illness self-care and the family lives of older adults: A synthetic review across four ethnic groups. Journal of Cross Cultural Gerontology, 25, 21-43. doi: 10.1007/s10823-0109112-z

Galvan, F.H. \& Caetano, R. (2003). Alcohol use and related problems among ethnic minorities in the United States. Alcohol Research and Health, 27, 87-94.

Garcia, C. (2005). Buscando trabajo: Social networking among immigrants from Mexico to the United States. Hispanic Journal of Behavioral Sciences, 27, 3-23.

Gfroerer J, \& De La Rosa, M. (1993). Protective and risk factors associated with drug use among Hispanic youth. Journal of Addictive Diseases. 12, 87-107.

Gfroerer, J.C. \& Tan, L.L. (2003). Substance use among foreign-born youths in the United States: Does length of residence matter? American Journal of Public Health, 93, 892-1895.

Gil, A. G., \& Vega, W. A. (2001). Latino drug use, scope, risk factors and reduction strategies. In M. Aguirre-Molina, C. W. Molina \& R. E. Zambrana (Eds.), Health issues in the Latino community (pp. 435-458). San Francisco: Jossey-Bass.

Gil, A.G. \& Vega, W.A. (1996). Two different worlds: Acculturation stress and adaptation among Cuban and Nicaraguan families. Journal of Social and Personal Relationships, 13, 435-456.

Gil, A.G., Wagner, E.F., \& Tubman, J.G. (2004). Culturally sensitive substance abuse intervention for Hispanic and African American adolescents: Empirical examples 
from the Alcohol Treatment Targeting Adolescents in Need (ATTAIN) Project. Addiction, 99(suppl2), 140-150.

Gillum, M.S., \& Holt, C.L. (2010). Association between religious involvement and behavioral risk factors of HIV/AIDS in Women and Men in a national health survey. Annals of Behavioral Medicine, 40, 284-293. doi 10.1007/s12160-0109218-0

Gloria, A. M., \& Peregoy, J. J. (1996). Counseling Latino alcohol and other substance users/abusers: Cultural considerations for Counselors. Journal of Substance Abuse Treatment, 13, 119-126. doi: 0740-5472/96

Gomez, C. A., Hernandez, M., \& Faigeles, B. (1999). Sex in the new world: An empowerment model for HIV prevention in Latina immigrant women. Health Education and Behavior, 26, 200-212.

Gonzalez-Morkos, B. (2005). Preliminary reliability analysis of a Spanish translation of the Brief RCOPE. Dissertation Abstracts International: Section B: The Sciences and Engineering, 66(10-B), 5680.

Gonzalez-Whippler, M. (2001). Santeria: The Religion. St. Paul, MN: Llewellyn.

Gorsuch, R. L. (1993). Assessing spiritual variables in Alcoholics Anonymous research. In B. S. McCrady \& W. R. Miller (Eds.), Research on Alcoholics Anonymous: Opportunities and alternatives (pp. 301-318). New Brunswick, NJ: Rutgers Center on Alcohol Studies.

Grewal, R., Cote, J.A., \& Baumgartner, H. (2004). Multicollinearity and measurement error in structural equation models: Implications for theory testing. Marketing Science, 23, 519-529. doi: $10.1287 / \mathrm{mksc} .1040 .0070$

Guarnaccia, P.J., P. Parra, A. Deschamps, G. Milstein, and N. Argiles. (1992). Si Dios quiere: Hispanic families' experiences of caring for a seriously mentally ill family member. Culture, Medicine, and Psychiatry 16, 187-215.

H., Palinkas, L. A., \& Edelman, S. V. (2010). Ethnic differences in complementary and alternative medicine use among patients with diabetes. Complementary Therapies in Medicine, 18, 241-248. doi: 10.1016/j.ctim.2010.09.007

Hair, J.F., Black, B., Babin, B., Anderson, R.E., \& Tatham, R.L. (2006). Multivariate Data Analysis, Sixth Edition. Pearson Prentice Hall: Upper Saddle River, NJ.

Hancock, G.R., \& Mueller, R.O. (2001). Rethinking construct reliability within latent variable systems. In R. Cudeck, S. du Toit, D. Sorebom (Eds.), Structural equation modeling: Present and future - a festschrift in honor of Karl Jöreskog, (pp. 195-21). Lincolnwood, IL: Scientific Software International. 
Harrison, M.O., Koenig, H.G., Hays, J.C., Eme-Akwari, A.G., \& Pargament, K. (2001). The epidemiology of religious coping: A review of the literature. International Review of Psychiatry, 13, 86-93.

Hasnain, M., Sinacore, J.M., Mensah, E.K., \& Levy, J.A. (2005). Influence of religiosity on HIV risk behaviors in active injection drug users. AIDS Care: Psychological and Socio-medical Aspects of AIDS/HIV, 17, 892-901. doi: $10.1080 / 09540120500038280$

Herrera, A.P., Lee, J.W., Nanyonjo, R.D., Laufman, L.E., \& Torres-Vigil, I. (2009). Religious coping and caregiver well-being in Mexican-American families. Aging \& Mental Health, 13, 84-91.

Hilfinger, M. Deanne, K, Rubio, M. (2004). Immigration and Health. Annual Review of Nursing Research, 22, 101-134.

Hill, P.C., \& Pargament, K.I. (2008). Advances in the conceptualization and measurement of religion and spirituality: Implications for physical and mental health research. Psychology of Religion and Spirituality, 1, 3-17.

Hill, T.D., Burdette, A., Ellison, C., \& Musick, M. (2006). Religious attendance and the health behaviors of Texas adults. Preventive Medicine, 42, 309-312.

Hill,T. D., Angel,J. L., Ellison, C. G., \& Angel R. J. (2005). Religious attendance and mortality: An 8-year follow-up of Mexican Americans. Journal of Gerontology, Series B: Psychological Sciences and Social Sciences, 60, S102-S109.

Hines, A. M \& Caetano, R. (1998). Alcohol and AIDS sexual behavior among Hispanic: Acculturation and gender differences. AIDS Education \& Prevention, 10, 533547.

Hodge, D.R. (2006) A template for spiritual assessment: A review of the JCAHO requirements and guidelines for implementation. Social Work, 51, 317-326.

Hodge, D.R. (1996). Religion in America: The demographics of belief and affiliation. In Shafranske (Ed.), Religion and the clinical practice of psychology (pp21-41). Washington DC: American Psychological Association.

Hodge, D.R., Andereck, K., Montoya, H. (2007). The protective influence of spiritualreligious lifestyle profiles on tobacco use, alcohol use, and gambling. Social Work Research, 31, 211-219.

Hoffman, N. A. (2011). The requirements for culturally and linguistically appropriate services in health care. Journal of Nursing Law, 14, 49-57. doi: 10.1891/10737472.14.2.49 
Holt, C.L., Haire-Joshu D.L., Lukwago, S.N., Lewellyn, L.A., Kreuter, M.W. (2005). The role of religiosity in dietary beliefs and behaviors among urban African American women. Cancer Control, 12, 84-90.

Honaker J., Joseph, A., King, G., Scheve, K., Singh, N., \& Amelia A. (2003). A program for missing data. Department of Government, Harvard University.

Horn, J.L., \& McArdle, J.J. (1992). A practical and theoretical guide to measurement invariance in aging research. Experimental Aging Research, 18, 117-144.

Hough, R., Karno, M., Burnam, M.A., Escobar, J.I. \& Timbers, D.M. (1983). The Los Angeles epidemiologic catchment area research program and the epidemiology of psychiatric disorders among Mexican Americans. Journal of Psychiatry, 14, 4251.

Hovey, J. D., \& Magana, C. (2000). Acculturative stress, anxiety, and depression among Mexican farm workers in the Midwest United States. Journal of Immigrant Health, 2, 119-131.

Interian, A., \& Diaz-Martinez, A.M. (2007). Considerations for culturally cognitivebehavioral therapy for depression with Hispanic patients. Cognitive and Behavioral Practice, 14, 84-97.

Jaccard, J., \& Wan, C. K. (1996). USREL approaches to interaction effects in multiple regression. Thousand Oaks, CA: Sage.

Jaccard, J., Turrisi, R., \& Wan, C. K. (1990). Interaction effects in multiple regression. Newbury Park, CA: Sage.

Jackson, D.L. (2003). Revisiting sample size and the number of parameters estimates: Some support for the N:q hypothesis. Structural Equation Modeling, 10, 128141.

Jackson, D.L. (2006). The effect of the number of observation per parameter in misspecified confirmatory factor analytic models. Structural Equation Modeling, 14, 48-76.

Jemmott, L.S., Jemmott, J.B. 3rd, \& Villarruel, A.M. (2002). Predicting intentions and condom use among Latino college students. Journal of the Association of Nurses in AIDS Care, 13, 59-69.

Kail, B. \& Elberth, M. (2003). Engaging and treating the substance-abusing Latina. Journal of Ethnicity in Substance Abuse, 2, 19-30.

Kazdin, A.E. (Ed.) (2000). Encyclopedia of psychology. London: Oxford University Press 
Kendler, K., Liu, X, Gardner, C., McCullough, M., Larson, D., \& Prescott, C. (2003). Dimensions of religiosity and their relationship to lifetime psychiatric and substance use disorders. American Journal of Psychiatry, 160, 496-503.

King, S.M. (2007). Religion, spirituality, and the workplace: Challenges for public administration. Public Administration Review, Jan./Feb., 103-113.

Kinsler, J.J., Lee. S., Sayles, J.N., Newman, P.A., Diamant, A. \& Cunningham, W. (2009). The impact of acculturation on utilization of HIV prevention services and access to care among an at-risk Hispanic population. Journal of Health Care for the Poor and Underserved, 20, 996-1011.

Kline, R.B. (2005). Principles and practice of structural equation modeling (2nd ed.). New York: Guilford.

Koenig, H.G. (1998). Handbook of religion and mental health. San Diego: Academic.

Koenig, H.G., McCullough, M.E., \& Larson, D.B. (2001). Handbook of religion and health. New York. Oxford University Press.

Lara, M., Gamboa, C., Kahramanian, M.I., Morales, L.S., \& Hayes Bautista, D.E. (2005) Acculturation and Latino health in the United States: A review of the literature and its sociopolitical context. Annual Review of Public Health, 26, 367-397. doi: 10.1146/annurev.publhealth.26.021304.144615.

Larkey, L.K., Hecht, M.L., Miller, K., \& Alatorre, C. (2001). Hispanic cultural norms for health-seeking behaviors in the face of symptoms. Health Education \& Behavior, 28, 65-80.

Lazarus, R.S. \& Folkman, S. (1984). Stress, appraisal, and coping. New York: Springer.

Lefkowitz, E.S., Gillen, M.M., Shearer, C.L., Boone, T.L. (2004). Religiosity, sexual behaviors, and sexual attitudes during emerging adulthood. Journal of Sex Research, 41, 150-159.

Levin, J. S., Markides, K. S., \& Ray, L. A. (1996). Religious attendance and psychological well-being in Mexican Americans: A panel analysis of threegenerations data. Gerontologist, 36, 454-463.

Levy, V., Page-Shafer, K., Evans, J., Ruiz, J., Morrow, S., Reardon J.,...McFarland, W. (2005). HIV-related risk behavior among Hispanic immigrant men in a population-based household survey in low-income neighborhoods of Northern California. Sexually Transmitted Diseases, 32, 487-490. 
Lewis-Coles, M.E. \& Constantine, M.G. (2006). Racism-related stress, Africultural coping, and religious problem-solving among African Americans. Cultural Diversity and Ethnic Minority Psychology, 12, 433-443.

Lieber, C.S. (2001). Liver diseases by alcohol and hepatitis C: Early detection and new insights in pathogenesis lead to improved treatment. American Journal on Addictions 10(Suppl.), 29-50.

Lipsky, S., \& Caetano, R. (2009). Epidemiology of substance abuse among Latinos. Journal of Ethnicity in Substance Abuse, 8, 242-260.

Little, T. D., Card, N. A., Bovaird, J. A., Preacher, K. J., \& Crandall, C. S. (2007). Structural equation modeling of mediation and moderation with contextual factors. In T. D. Little, J. A. Bovaird, \& N. A. Card (Eds.), Modeling contextual effects in longitudinal studies (pp. 207-230). Mahwah, NJ: Lawrence Erlbaum.

Lobell, M. Bay, C., Rhoads, K., \& Keske, B. (1994). Barriers to cancer screening in Hispanic women. American Society of Clinical Oncology, 13, 169.

Loue, S., Cooper, M., \& Fiedler, J. (2003). HIV knowledge among a sample of Puerto Rican and Mexican men and women. Journal of Immigrant Health, (5), 59-65.

Loury, S., \& Kulbok, P. (2007). Correlates of alcohol and tobacco use among Mexican immigrants in rural North Carolina. Farm Community Health, 30, 247-256.

Lucas, I. (1981). The browning of America: The Hispanic revolution in the American church. Chicago: Fides/Claretian.

Lueck, K. \& Wilson, M. (2011). Acculturative stress in Latino immigrants: The impact of social, socio-psychological and migration-related factors. International Journal of Intercultural Relations, 35, 186-195.

Lundgren, L., Chassler, D., Ben-Ami, L., Schilling, R., \& Purington, T. (2005). Factors associated with emergency room use among injection drug users of African American, Hispanic, and While European Background. The American Journal of Addictions, 14, 268-280.

MacCallum, R.C., Browne, M.W., \& Sugawara, H.M. (1996). Power analysis and determination of sample size for covariance structure modeling. Psychological Methods, 1, 130-149.

Magaña, A. \& Clark, N.M. (1995). Examining a paradox: Does religiosity contribute to positive birth outcomes in Mexican American populations. Health Education \& Behavior, 22, 96-109. 
Magaña, J.R., de la Rocha, O., Amsel, J., Magana, H.A., Fernandez, M.I., \& Rulnick, S. (1996). Revisiting the dimensions of acculturation: Cultural theory and psychometric practice. Hispanic Journal of Behavioral Sciences, 18, 444-468.

Marín, B.V. \& Marín, G. (1992). Predictors of condom accessibility among Hispanics in San Francisco. American Journal of Public Health, 82, 592-595.

Marín, G. (1992). Issues in the measurement of acculturation among Hispanics. In KF Geisinger (Eds.), Psychological Testing of Hispanics (pp. 23-51). Washington, DC: American Psychological Association.

Marín, G., Gamba, R.J. (1996). Issues in the measurement of acculturation among Hispanics. In K.F. Geisinger (Eds.), Psychological Testing of Hispanics (pp. 23-51). Washington, DC: American Psychological Association.

Marín, G., Sabogal, F., Marín, B.V., Otero-Sabogal, R., \& Perez-Stable, E.J. (1987). Development of a short acculturation scale for Hispanics. Hispanic Journal of Behavioral Science, 9, 183-205.

Marín. G. \& Posner, S.F. (1995). The role of gender and acculturation on determining the consumption of alcoholic beverages among Mexican-Americans and Central Americans in the United States. International Journal of the Addictions, 30, 779794.

Markides, K. S., Levin,J. S., \& Ray, L. A. (1987). Religion, aging, and life satisfaction: An eight-year, three-wave longitudinal study. Gerontologist, 21, 660-678.

Markides, K.S., Krause, N., Mendes de Leon, C.F. (1988). Acculturation and alcohol consumption among Mexican Americans: A three-generational study. American Journal of Public Health, 78, 1178-1181.

Marler, P. L., \& Hadaway, C. K. (2002). Being religious or being spiritual in America: A zero- sum proposition? Journal for the Scientific Study of Religion, 41, 289300

Marsh, H.W., Balla, J.R., \& McDonald, R.P. (1988). Goodness-of-fit indexes in confirmatory factor analysis: The effect of sample size. Psychological Bulletin, 103, 391-410.

Martens, M. P. (2005). The use of structural equation modeling in counseling psychology research. The Counseling Psychologist, 33, 269-298.

Martinez, C.R, McClure, H.H., Eddy, J.M., \& Wilson, D.M. (2011). Time in U.S. residency and the social, behavioral, and emotional adjustment of Latino immigrant families. Hispanic Journal of Behavioral Sciences, 33, 323-349. 
Mausbach, B.T., Coon, D.W., Cardenas, V., \& Thompson, L.W. (2003). Religious coping among Caucasian and Latina dementia caregivers. Journal of Mental Health and Aging, 9, 97-100.

McConnell, K.M., Pargament, K.I., Ellison, C.G., \& Flannelly. (2006). Examining the links between spiritual struggles and symptoms of psychopathology in a national sample. Journal of Clinical Psychology, 62, 1469-1484. doi: 10.1002/jclp.20325

McDonald, R. P., \& Ho, R. (2002). Principles and practice in reporting structural equation analyses. Psychological Methods, 7, 64-82.

McKeever, M \& Klineberg, S.L. (1999). Generational differences in attitudes and socioeconomic status among Hispanics in Houston. Sociological Inquiry, 69, 33.

McNeill, B. W., \& Cervantes, J. M. (2008). Counselors and curanderas/os: Parallels in the healing process. In B. W. McNeil \& J. M. Cervantes (Eds.), Latina/o Healing Practices: Mestizo and Indigenous Perspectives (pp. xvii-xxxiii). New York: Routledge.

Menagi, F.S., Harrell, Z. \& June, L.N. (2008). Religiousness and college student alcohol use: Examining the role of social support. Journal of Religion \& Health, 47, 217226.

Mendoza, R.H. (1989). An empirical scale to measure type and degree of acculturation in Mexican-American adolescents and adults. Journal of Cross Cultural Psychology, 20, 372-385.

Meredith, W. (1993). Measurement invariance, factor analysis and factorial invariance. Psychometrika, 58, 535-543.

Miami-Dade County Health Department. (2011). HIV/AIDS among Hispanics Fact Sheet.

Michalak, L., Trocki, K., \& Bond, J. (2007). Religion and alcohol in the U.S. National Alcohol Survey: How important is religion for abstention and drinking? Drug and Alcohol Dependence, 87, 268-280.

Miller, W. R. (1998). Researching the spiritual dimensions of alcohol and other drug problems. Addiction, 93, 979-990. doi:10.1046/j.1360-0443.1998.9379793.x.

Miller, W. R., \& Thoresen, C. E. (2003). Spirituality, religion, and health: An emerging research field. American Psychologist, 58, 24-35. doi:10.1037/0003066X.58.1.24.

Miller, W.R (1999). Integrating spirituality into treatment: Resources for practitioners. Washington D.C.: American Psychological Association. 
Miner, M.H., \& McKnight, J. (1999). Religious attributions: Situational factors and effects on coping. Journal for the Scientific Study of Religion, 38, 274-286

Molina, C.W., Zambrana, R.E., \& Aguirre-Molina, M. (1994). The influence of culture, class, and environment on health care, in C.W. Molina \& M. Molina (Eds.), Latino Health in the U.S.: A Growing Challenge (pp. 23-43). Washington, DC: American Public Health Association.

Moody-Smithson, M. (2001). Religion's effects on health outcomes. A literature review. Presented at CSAT's Faith and Community Partners Initiative National Focus Group Meeting, July 26-27, 2001. Washington, D.C. Rockville, MD: Logicon/Row Sciences, Inc.

http://www.casacolumbia.org/absolutenm/templates/PressReleases.asp?articleid= $\underline{48}$.

Moos, R.H., \& Schaefer, J.A. (1982). Coping resources and processes: Current concepts and measures. In L. Goldberger \& S. Brenznitz (eds.), Handbook of stress: Theoretical and clinical aspects ( $2^{\text {nd }}$ ed., pp. 234-257). New York: The Free Press.

Moreno, C. L. (2007). Relationship between culture, gender, structural factors, abuse, trauma, and HIV/AIDS for Latinas. Qualitative Health Research, 17, 340-352.

Muthén, L.K. and Muthén, B.O. (2010). Mplus User's Guide. Sixth Edition. Los Angeles, CA: Muthén \& Muthén

National Association of Social Workers. (2010, August). Immigration and Refugee Resettlement. Retrieved April 5, 2011 from http://www.naswdc.org/pubs/news/2006/02/desilva.asp

National Center on Minority Health and Health Disparities. (2009). Vision and mission. Retrieved on June 16, 2009 from http://ncmhd.nih.gov/about ncmhd/mission.asp.

National Institute on Drug Abuse. (1993). Community Research Branch. Risk Behavior Assessment (3rd ed.). Rockville, MD: NIDA.

National Institute on Drug Use. (2006). HIV AIDS: How does drug use impact the HIV/AIDS Epidemic. Research Report Series. NIH Publication Number 06-5760.

Needle, R., Fisher D.G., Weatherby, N., Chitwood, D., Brown, B., Cesari, H.,...Braunstein, M. (1995). Reliability of self-reported HIV risk behaviors of drug users. Psychology of Addictive Behaviors, 9, 242-250.

Neff, J.A. \& Hoppe, S.K. (1993). Race/ethnicity, acculturation, and psychological distress: fatalism and religiosity as cultural resources. Journal of Community Psychology, 21, 3-20. 
Negy, C., \& Woods, D.J. (1992). The importance of between acculturation and socioeconomic status. Hispanics Journal of Behavioral Science, 14, 248-251.

Newcomb, M.D., Wyatt, G.E., Romero, G.J., Tucker, B.M., Wayment, H.A., Carmona, J.,...Mitchell-Kernan, C. (1998). Acculturation, sexual risk taking and HIV health promotion among Latinas. Journal of Counseling Psychology, 45, 454467.

Nyamathi, A., Bennet, C., Leake, B., Lewis, C., \& Flaskerud, J. (1993). AIDS-related knowledge, perceptions, and behaviors among impoverished minority women. American Journal of Public Health, 83, 65-71.

Ortiz, F. A., Davis, K. G., \& McNeill, B. W. (2008). Curanderismo: Religious and spiritual worldviews and indigenous healing traditions. In B. W. McNeil \& J. M. Cervantes (Eds.), Latina/o Healing Practices: Mestizo and Indigenous Perspectives (pp. 271-300). New York: Routledge.

Otero-Sabogal, R., Stewart, S., Sabogal, F., Brown, B.A., Perez- Stable, E.J. (2003). Access and attitudinal factors related to breast and cervical cancer rescreening: Why are Latinas still under screened? Health Education \& Behavior, 30, $337-$ 359.

Pardini, D., Plane, T.G., Sherman, A., \& Stump. J.E. (2000). Religious faith and spirituality in substance abuse recovery: Determining the mental health benefits. Journal of Substance Abuse Treatment, 19, 347-354. doi:10.1016/S07405472(00)00125-2.

Pargament, K.I. (2002b). The bitter and sweet: An evaluation of the costs and benefits of religiousness. Psychological Inquiry, 13, 168-181.

Pargament, K.I. (1997). The psychology of religion and coping: Theory, research, and practice. New York: Guilford Press.

Pargament, K.I. (2002a). Is religion nothing but...? Explaining religion versus explaining religion away. Psychological Inquiry, 13, 239-244.

Pargament, K.I., Ensing, D.S., Falgout, K., Olsen, H., Reilly, B., Van Haitsma, K., Warren. R. (1990). God help me: Religious coping efforts as predictors of the outcomes to significant negative life events. American Journal of Community Psychology, 18, 83-824.

Pargament, K.I., Kennell, J., Hathaway, W., Grevengoed, N., Newman, J., \& Jones, W. (1988). Religion and the problem solving process: Three styles of coping. Journal for the Scientific Study of Religion, 27, 90-104. 
Pargament, K.I., Koenig, H.G., \& Perez, L. (2000). The many methods of religious coping: Initial development and validation of the RCOPE. Journal of Clinical Psychology, 56, 519-543.

Pargament, K.I., Koenig, H.G., \& Tarakeshwar, N. (2001). Religious struggles as a predictor of mortality among medically ill elderly patients: A 2-year longitudinal study. Archives of Internal Medicine, 161, 1881-1885.

Pargament, K.I., Smith, B.W., Koenig, H.G., \& Perez, L. (1998). Patterns of positive and negative religious coping with major life stressors. Journal for the Scientific Study of Religion, 37, 710-724.

Park, C., Cohen, L. H., \& Herb, L. (1990). Intrinsic religiousness and religious coping as life stress moderators for Catholics versus Protestants. Journal of Personality and Social Psychology, 59, 562-574.

Pearlin, L.I. (1989). The sociological study of stress. Journal of Health and Social Behavior, 30, 241-256.

Pearlin, L.I., Lieberman, M.A., Menaghan, E.G.,\& Mullan, J.T. (1981). The stress process. Journal of Health and Social Behavior, 22, 237-256

Perez, M. C., \& Fortuna, L. (2005). Psychosocial stressors, psychiatric diagnosis, and utilization of mental health services among undocumented immigrant Latinos. Journal of Immigrant and Refugee Services, 3, 107-123.

Pew Resarch Center. (2009). U.S. population projections 2005-2050. Retrieved July 3, 2009 from http://pewhispanic.org/reports/report-php?RepostID=85.

Plante, T.G., \& Sherman, A.C. (Eds.). (2001). Faith and health: Psychological perspectives. New York: Guilford Press.

Plante, T.G., Maunel, G., Menendez, A., \& Marcotte, D. (1995). Coping with stress among Salvadoran immigrants. Hispanic Journal of Behavioral Sciences, 17, 471-479.

Prado, G., Feaster, D.J., Schwartz, S.J., Pratt, I.A., Smith, L., \& Szapocznik, J. (2004). Religious involvement, coping, social support, and psychological distress in HIVseropositive African American mothers. AIDS Behavior, 221-235.

Prado, G., Szapocznik, J., Maldonado-Molina, M.M., Schwartz, S., \& Pantin, H. (2008). Drug use/abuse prevalence, etiology, prevention, and treatment in Hispanic adolescents: A cultural perspective. Journal of Drug Issues, 38, 5-36.

Rabinowitz, Y.G., Hartlaub, M.G., Saenz, E.C., Thompson, L.W., \& GallagherThompson. (2010). Is religious coping associated with cumulative health risk? An examination of religious coping styles and health behaviors patterns in 
Alzheimer's dementia caregivers. Journal of Religion and Health, 49, 498-512. doi: $10.1007 / \mathrm{s} 10943-009-9300-8$

Redfield, R., Linton, R., Herskovits, M.J. (1936). Memorandum for the study of acculturation. American Anthropologist, 36, 149-152.

Ritt-Olson, A., Milam, J., Unger, J. B., Trinidad, D., Teran, L., Dent, C. W., \& Sussman, S. (2004). The protective influence of spirituality and health-as-a-value against monthly substance use among adolescents varying in risk. Journal of Adolescent Health, 34, 192-199.

Robbins L.N. \& Regier, D.A. (1991). Psychiatric disorders in America: The epidemiological Catchment Area Study. New York: Free Press.

Robinson, E., Cranford, J.A., Webb, J.R., \& Brower, K.J. (2007). Six-month changes in spirituality, religiousness, and heavy drinking in a treatment-seeking sample. Journal of Studies on Alcohol and Drugs, 68, 282-290.

Rojas-Guyler, L., Ellis, N., \& Sanders, S. (2005). Acculturation, health protective sexual communication, and HIV/AIDS risk behavior among Hispanic women in a large midwestern city. Health Education \& Behavior, 32, 767-779.

Romero, G. J., Arguelles, L., \& Rivero, A. (1993). Latinas and HIV infection/AIDS: Reflections on impacts, dilemmas and struggles. In B. Blair \& S. Cayleff (Eds.), Wings of gauze: Women and the experience of illness (pp. 340-352). Detroit, MI: Wayne State University Press.

Rote, S.M., \& Starks, B. (2010). Racial/ethnic differences in religiosity and drug use. Journal of Drug Issues, 40, 729-754

Rudmin, F. (2009). Constructs, measurements and models of acculturation and acculturative stress. International Journal of Intercultural Relations, 33, 106-123.

Ruiz, P. (2002). Commentary: Hispanic access to health/mental health services. Psychiatric Quarterly, 73, 85-91.

Sabogal, F., Marin, G., Otero-Sabogal, R. (1987). Hispanic familism and acculturation: What changes and what doesn't? Hispanic Journal of Behavioral Sciences, 9 , 397-412.

Sabogal, F., Perez-Stable, E., \& Otero-Sabogal, R. (1995). Gender, ethnic and acculturation differences in sexual behaviors: Hispanic and non- Hispanic White adults. Hispanic Journal of Behavioral Sciences, 17, 139-159.

Salganik, M.J. \& Heckathorn, D.D. Sampling and estimation in hidden populations using respondent-driven sampling. Sociological Methodology, 34, 193-240. 
Scarinci, E.G., Griffin, M.T., Grogoriu, A., \& Fitzpatrick, J.J. (2009). Spiritual wellbeing and spiritual practices in HIV-infected women: A preliminary study. Journal of the Association of Nurses in AIDS Care, 20, 69-76.

Schwartz, S.J. \& Zamboango, B.L. (2008). Testing Berry's Model of acculturation: A confirmatory latent class approach. Cultural Diversity and Ethnic Minority Psychology, 14, 275-285.

Schwartz, S.J., Unger, J., Zamboanga, B.L., \& Szapocznik, J. (2010). Rethinking the concept of acculturation: Implications for theory and research. American Psychologist, 65, 237-251. doi: 10.1037/a0019330

Schwartz, S.J., Waterman, A.S., Vazsonyi, A.T., Zamboanga, B.L., Whitbourne, S.K., Weisskirch, R.S...Ham., L.S. (2011). The association of well-being with health risk behaviors in college-attending youth adults. Applied Developmental Science, 15, 20-36. doi: 10.1080/10888691.2011.538817.

Scribner, R. (1996). Paradox as paradigm: The health outcomes of Mexican Americans. American Journal of Public Health, 86, 303-305.

Seeman, T.E., Dubin, L.F., \& Seeman, M. (2003). Religiosity/spirituality and health: A critical review of the evidence for biological pathways. American Psychologist, $58,53-63$.

Seybold, K.S., \& Hill, P.C. (2001). The role of religion and spirituality in mental and physical health. Current Directions in Psychological Science, 10, 21-24.

Shedlin, M.G., Ulises, C., \& Oliver-Velez, D. (2005). Initial acculturation and HIV risk among new Hispanic immigrants. Journal of the National Medical Association, 97, 32S-37S.

Shorkey, C., Uebel, M., Windsor, L.C. (2008). Measuring dimensions of spirituality in chemical dependence treatment and recovery: Research and practice. International Journal of Mental Health \& Addiction, 6, 286-305. doi: 10:1007/s11469-9065-9.

Simons, L.G., Burt, C.H., Peterson, F.R. (2009). The effect of religion on risky sexual behavior among college students. Deviant Behavior, 30, 467-485.

Skinner, D.G., Correa, V., Skinner, M., \& Baily, D.B. (2001). The role of religion in the lives of Latino families of young children with developmental delays. American Journal of Mental Retardation, 106, 297-313.

Smiley, M. R., McMillan, S. C., Johnson, S., \& Ojeda, M. (2000). Comparison of Florida Hispanic and Non-Hispanic Caucasian women in their health belief related to breast cancer and health locus of control. Oncology Nurse Forum, 27, 975-984. 
Sobell, L.C., \& Sobell, M.B. (1992). Timeline followback: A technique for assessing self-reported alcohol consumption. In R.Z. Litten \& J. Allen (Eds.), Measuring alcohol consumption: Psychosocial and biological methods (pp. 41-72). New Jersey: Humana Press.

Steinbauer, J.R., Cantor, S.B., Holzer, C.E, Volk, R.J. (1998). Ethnic and sex bias in primary care screening tests for alcohol use disorders. Annals Internal Medicine, 129, 353- 362.

Sterk, C.E., Klein, H, Elifson, K.W. (2004). Predictors of condom-related attitudes among at-risk women. Journal of Women's Health,13, 676-688.

Stinson, F., Grant, B., Dufour, M. (2001). The critical dimension of ethnicity in liver cirrhosis mortality statistics. Alcoholism: Clinical \& Experimental Research, 25, $1181-1187$.

Substance Abuse and Mental Health Services Administration, Office of Applied Studies. (2007). The NSDUH Report: Religious Involvement and Substance Use among Adults. Rockville, MD.

Szymanski, D.M. \& Obiri, O. (2010). Do religious coping styles moderate or mediate the external and internalized racism-distress links? The Counseling Psychologist, 39, 438-462. doi: 10.1177/0011000010378895

Tanaka, J.S. (1987). How much is big enough?: Sample size and goodness of fit in structural equation models with latent variables. Child Development, 58, 134146.

Tarakeshwar, N., Stanton, J., \& Pargament, K.I. (2003). Religion: An overlooked dimension in cross-cultural psychology. Journal of Cross-Cultural Psychology, 34, 377-394. doi: 10.1177/0022022103034004001

Taylor, R.J., Chatters, L.M., \& Levin, J.S. (2004). Religion in the Lives of African Americans. Thousand Oaks, CA: Sage.

Thoresen, C. E. (1998). Spirituality, health and science. In S. Roth- Roemer, S. R. Kurpius, \& C. Carmin (Eds.), The emerging role of counseling psychology in health care (pp. 409-431). New York: Norton.

Tix, A.P. \& Frazier, P.A. (1998). The use of religious coping during stressful life events: Main effects, moderation, and mediation. Journal of Counseling and Clinical Psychology, 66, 411-422.

Turner, R.J. \& Avision, W.R. (2003). Status variations in stress exposure: Implications for the interpretation of research on race, socioeconomic status, and gender. International Journal of Intercultural Relations, 44, 488-505. 
Turner, R.J., Lloyd, D.A, \& Taylor, J. (2005). Stress burden, drug dependency and the nativity paradox among U.S. Hispanics. Drug and Alcohol Dependence, 83, 7989. doi: $10.1016 / /$ j.drugalcdep.2005.11.003.

U.S. Census Bureau. (2011). The Hispanic Population: 2010 Census Briefs.

U.S. Department of Health and Human Services, Office of Minority Health. (2001). National standards for culturally and linguistically appropriate services (CLAS) in health care. Federal Registry, 65(247), 80865-80879.

U.S. Department of Health and Human Services. (2009). Substance abuse among Latinos: Organizations help on the Path to Recovery. Retrieved on June 11, 2009 from http://www.omhrc.gov/npa/templates/content.aspx?!ID=52\&lvl=2\&lvllD=4.

Ullman, J.B. (1996). Structural equation modeling. In B.G. Tabachnick \& L.S. Fidell (Eds.), Using multivariate statistics (pp. 709-811). New York: Harper Collins.

Valle, R. (1994). Culture-fair behavioral symptom differential assessment and intervention in dementing illness. Alzheimer Disease \& Associates Disorders, 8(Suppl. 3), 21-45.

VanOss Marin, B. (2003). HIV prevention in the Hispanic community: Sex, culture, and empowerment. Journal of Transcultural Nursing, 14, 186-192.

Vega, W. (1980). The Hispanics natural healer: A case study. In R. Valle \& . Vega (Eds.), Hispanic Natural Support Systems (N80-620047). Sacramental, CA: Department of Mental Health.

Vega, W. A. \& Sribney, W. M. (2005). Seeking care for alcohol problems: Patterns of need and treatment among Mexican-origin adults in central California. Alcoholism Treatment Quarterly, 23, 29-51.

Vega, W.A. \& Gil, A.G. (1998). Drug use and ethnicity in early adolescence. New York: Plenium Press.

Vega, W.A., \& Amaro, H. (1994). Latino outlook: Good health, uncertain prognosis. Annual Review of Public Heatlh, 15, 39-67.

Vega, W.A., Alderete, E., Kolody, B., \& Aguilar Gaxiola, S. (1998). Illicit drug use among Mexican and Mexican Americans in California: The effects of gender and acculturation. Addiction, 93, 1839-1850.

Vega, W.A., Kolody, B., Hwang, J., Noble, A., \& Poret, P.A., (1997). Perinatal drug use among immigrant and native-born Latinas. Substance Use \& Misuse, 32, 43-62. 
Velez, C.N. \& Ungemack, J.A. (1989). Drug use among Puerto Rican youth: An exploration of generational status differences. Social Science \& Medicine, 29, 779-789.

Viladrich, A., \& Abraido-Lanza, A. F. (2009). Religion and mental health among minorities and immigrants in the U.S. In S. Loue \& M. Sajatovic (Eds.), Determinants of Minority Health and Wellness (pp. 149-174). doi: 10.1007/9780-387-75659-2 8

Villa-Caballero, L., Morello, C. M., Chynoweth, M. E., Prieto-Rosinol, A., Polonsky, W. Palinkas, L.A., \& Ede;man, S.V. (2010). Ethnic differences in complementary and alternative medicine use among patients with diabetes. Complementary Therapies in Medicine, 18, 241-248.

Wagner, E.F. (2003). Conceptualizing alcohol treatment for Hipnic/Latino adolescents. Alcoholism: Clinical and Experimental Research, 27, 1349-1352.

Wallace, K. A., \& Bergman, C. S. (2002). Spirituality and religiosity in a sample of African American elders: A life story approach. Journal of Adult Development, 9,141-154.

Wallerstein, N.B. \& Duran, B. (2006). Using Community-Based Participatory Research to Address Health Disparities. Health Promotion Practice, 7, 312.

Wang, S.C., Schwatrz, S.J., \& Zamboanga, B. (2010). Acculturative stress among Cuban American college students: Exploring the mediating pathways between acculturation and psychosocial functioning. Journal of Applied Social Psychology, 40, 2862-2887.

Waters, J. A., Fazio, S. L., Hernandez, L., \& Segarra, J. (2002). The Story of CURA, a Hispanic/Latino Drug Therapeutic Community. Journal of Ethnicity in Substance Abuse, 1, 113-134.

Wayment, H.A., Wyatt, G.E., Tucker, M., Romero, G.J., Carmona, J.V., Newcomb, M.D.,...Mitchell-Kernan, C. (2003). Predictors of risky and precautionary behaviors among single and married white women. Journal of Applied Social Psychology, 33, 791-816. doi: 10.1111/j.1559-1816.2003.tb01925.x

Weatherby, N.L., Needle, R., Cesari, H., Booth, R., McCoy, C.B., Watters, J.K.,... Chitwood, D.D. (1994). Validity of self-reported drug use among injection drug users and crack cocaine users recruited through street outreach. Evaluation and Program Planning, 17, 347-355.

Weidel, J.J., Provenscio-Vazquez, E., Watson, S.D., \& Gonzalez-Guarda, R. (2008). Cultural considerations for intimate partner violence and HIV risk in Hispanics. Journal of the Association of Nurses in AIDS Care, 19, 247-251. 
Whiteside A. (2008). HIV/AIDS: A very short introduction. Oxford, UK: Oxford University Press.

Wothke, W. (2000). Longitudinal and multi-group modeling with missing data. In T. D. Little, K. U. Schnabel, \& J. Baumert (Eds.) Modeling longitudinal and multiple group data: Practical issues, applied approaches and specific examples. Mahwah, NJ: Erlbaum.

Wyatt, G.E., Carmona, J.V., Loeb, T.B., Guthrie, D., Chin, D., \& Gordon, G. (2000). Factors affecting HIV contraceptive decision-making among women. Sex Roles, 42, 495-521. doi: 10.1023/A: 1007091121084

Yakushko, O., Watson, M., \& Thompson, S. (2008). Stress and coping in the lives of recent immigrant and refugees: Consideration for counseling. International Journal for the Advancement of Counseling, 30, 167-178. doi: 10.1007/s10447008-9054-0

Zea, M.C., Asner-Self, K.K., Birman, D., \& Buki, L.P. (2003). The Abbreviated Multidimentional Acculturation Scale: Empirical validation with two Latino/Latina samples. Cultural Diversity and Ethnic Minority Psychology, 9, $107-126$.

Zemore, S.E., Mulia, N., Ye, Y., Borges, G., \& Greenfield, T.K. (2009). Gender, acculturation, and other barriers to alcohol treatment utilization among Latinos in three National Alcohol Surveys. Journal of Substance Abuse Treatment, 36, 446456. doi: $10.1016 /$ j.sat.2008.09.005

Zinnbauer, B. J., Pargament, K. I., Cole, B., Rye, M. S., Butter, E. M., Belavich, T. G.,...Kadar, J.L. (1997). Religion and spirituality: Unfuzzying the fuzzy. Journal for the Scientific Study of Religion, 36, 549-564. 
APPENDICES 


\section{Appendix A}

\section{Risk Behavior Survey}

\{Module Name\} Module

\section{Risk Behavior Survey}

Agency Name:

ID\#:
Site Name:

Date: $--1+-1---1$

The RBS contains sensitive information on drug use and sexual behavior. Please ensure that you have developed rapport with the participant before asking these questions.

A. DRUG USE

\begin{tabular}{|c|c|c|c|c|c|c|c|c|c|c|}
\hline & & & 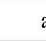 & . & & b. & c. & d. & e. & f. \\
\hline & $\begin{array}{l}\text { going to ask you some } \\
\text { ons about your drug use. } \\
\text { ask what types of drugs } \\
\text { ve used and how often } \\
\text { you use them. }\end{array}$ & & $\begin{array}{l}\text { Te you } \\
\text { ouse, } \\
\text { ed skip }\end{array}$ & $\begin{array}{l}\text { ever use } \\
\text { inknown, } \\
\text { to next d }\end{array}$ & & $\begin{array}{l}\text { How many days did } \\
\text { you use _ in the last } \\
30 \text { days? } \\
\text { (ffo, do not ask purts c-f, } \\
\text { and skip to next trugg) }\end{array}$ & $\begin{array}{c}\text { How many days did you } \\
\text { inject_ in the last } 30 \\
\text { days? } \\
\text { (If } 0 \text {, ship to parte) }\end{array}$ & $\begin{array}{c}\text { How many times a } \\
\text { day did you inject } \\
? \\
\text { (Average } \# \text { of } \\
\text { injections idgy) }\end{array}$ & $\begin{array}{l}\text { How many days did you } \\
\text { use without injecting } \\
\text { (smoking, snorting, } \\
\text { swallowing) in the last } 30 \\
\text { days? } \\
\text { (If } 0 \text {, then ship to next drug) }\end{array}$ & $\begin{array}{l}\text { How many times a } \\
\text { day did you use } \\
\text { without injecting? }\end{array}$ \\
\hline & & \begin{tabular}{|l|} 
YES \\
\end{tabular} & NO & $\overline{\mathrm{INK}}$ & REF & & & & & \\
\hline 1. & $\begin{array}{l}\text { Cocaine by itself } \\
\text { (injected or snorted) }\end{array}$ & a & ] & J & $\square$ & -- & -- & -- & -- & -- \\
\hline 2. & Heroin by itself & 0 & a & a & $\square$ & -- & -- & -- & -- & -- \\
\hline 3. & $\begin{array}{l}\text { Heroin \& Cocaine } \\
\text { mixed together } \\
\text { (Speedball) }\end{array}$ & a & $\square$ & ] & $\square$ & -- & -- & -- & -- & -- \\
\hline 4. & $\begin{array}{c}\text { Other Opiates } \\
\text { (Demerol, Codeine, } \\
\text { Dilaudid) }\end{array}$ & $\square$ & ] & J & व & -- & -- & -- & -- & -- \\
\hline 5. & $\begin{array}{l}\text { Amphetamines } \\
\text { (Speed, } \\
\text { Methamphetamine, } \\
\text { Crank) }\end{array}$ & D & 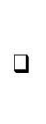 & ] & $\square$ & -- & -- & -- & -- & -- \\
\hline
\end{tabular}


B. DRUG INJECTION (if no injection use in past 30 days, skip to Section C)

1. In the last 30 days, how many times (\# of injections) did you inject using works (needle/syringes) that you know had been used by somebody else? If none, enter 000 and skip to B3.

2. Of the times you injected after someone, how many times did you clean the works with full-strength bleach? Number cannot exceed total number of times used after somebody else (B1).

3. How many times in the last 30 days did you use a cooker/cotton/rinse water that had been used by another injector?

4. How many times in the last 30 days did you fix drugs with another person, then split the drug solution (through use of the same cooker/spoon or through front or back loading)?

\section{SEXUAL ACTIVITY} Now I'm going to ask you some questions about sex. I'm referring here to anybody you've had sex with in the last 30 days.

1. During the last 30 days, with how many people did you have vaginal, oral or anal sex? IF NONE, ENTER 000 AND THE QUESTIONNAIRE IS COMPLETED.

2. How many of your partners were female? Number cannot exceed total number of people (Cl).

3. How many of your partners were male? Number cannot exceed total number of people (CI).

4. Interviewer: Code gender of respondent

Male $\square 1$ If Male, complete sections $D, E, F, G \& I$ If Female, complete sections $D, G, H \& I$

If Don't Know, ask ALL sex/gender specific questions and allow client to answer as they like.

Use the following coding for frequency of sexual events and condombarrier use.

$\begin{array}{llll}\text { Frequency scale: } & & \text { Condom/Barrier use scale: } \\ \text { Once or irregularly } & 01 & \text { Never } & 0 \\ \text { Less than once a week } & 02 & \text { Less than half the time } & 1 \\ \text { About once a week } & 03 & \text { About half the time } & 2 \\ \text { 2-6 times a week } & 04 & \text { More than half the time } & 3 \\ \text { About once a day } & 05 & \text { Always } & 4 \\ \text { 2-3 times a day } & 06 & \text { Don't know/unsure } & -2 \\ \text { 4 or more times a day } & 07 & \text { Refused } & -1 \\ \text { Don't know/unsure } & -2 & & \\ \text { Refused } & -1 & & \end{array}$

\section{Ask Male/Female/Gender Unknown Clients who had Female Partners}

1a. How many women performed oral sex ("went down") on you?

If 0 , then skip to question $2 a$. Number cannot exceed total number of female partners (C2).

1b. How often did your partner(s) perform oral sex ("go down") on you? (Use frequency scale)

1c. How often did you use condoms/dental dams when your partner(s) performed oral sex ("went down") on you? (Use condom/barrier use scale)

2a. How many women did you perform oral sex ("go down") on?

If 0, then skip to next section appropriate for the sex of this client. Number cannot exceed total number of female partners (C2).

2b. How often did you perform oral sex ("go down") on your partner(s)? (Use frequency scale)

2c. How often did you use condoms/dental dams when you performed oral sex ("went down") on your partner(s)? (Use condom/barrier use scale) 


$\begin{array}{lr}\text { Condom/Barrier use scale: } \\ \text { Never } & 0 \\ \text { Less than half the time } & 1 \\ \text { About half the time } & 2 \\ \text { More than half the time } & 3 \\ \text { Always } & 4 \\ \text { Don't know/unsure } & -2 \\ \text { Refused } & -1 \\ \end{array}$

$\begin{array}{ll}\text { Frequency scale: } & \\ \text { Once or irregularly } & 01 \\ \text { Less than once a week } & 02 \\ \text { About once a week } & 03 \\ \text { 2-6 times a week } & 04 \\ \text { About once a day } & 05 \\ \text { 2-3 times a day } & 06 \\ \text { 4 or more times a day } & 07 \\ \text { Don't know/unsure } & -2 \\ \text { Refused } & -1\end{array}$

E. Ask Male/Gender Unknown Clients who had Female Partners

1a. How many women did you have vaginal sex with?

If 0 , then skip to question $2 a$. Number cannot exceed total number of female partners (C2).

1b. How often did you have vaginal sex? (Use frequency scale)

1c. How often did you use a condom? (Use condom/barrier use scale)

2a. How many women did you have (insertive) anal sex with?

If 0 , then skip to next section appropriate for the sex of this client. Number cannot exceed total number of female partners $(C 2)$.

2b. How often did you have (insertive) anal sex? (Use frequency scale)

2c. How often did you use a condom? (Use condom/barrier use scale)

F. Ask Male/Gender Unknown Clients who had Male Partners

1a. How many men did you have (insertive) anal sex with?

If 0 , then skip to question $2 a$. Number cannot exceed total number of male partners (C3).

1b. How often did you have (insertive) anal sex? (Use frequency scale)

1c. How often did you use a condom? (Use condom/barrier use scale)

G. Ask Male/Female/Gender Unknown Clients who had Male Partners

1a. How many men performed oral sex ("went down") on you?

If 0 , then skip to question $2 a$. Number cannot exceed total number of male partners (C3).

1b. How often did your partner(s) perform oral sex ("go down") on you? (Use frequency scale)

1c. How often did you use condoms/dental dams when your partner(s) performed oral sex ("went down") on you? (Use condom/barrier use scale)

2a. How many men did you perform oral sex ("go down") on?

If 0 , then skip to next section appropriate for the sex of this client. Number cannot exceed total number of male partners (C3).

2b. How often did you perform oral sex ("go down") on your partner(s)? (Use frequency scale)

2c. How often did you use condoms/dental dams when you performed oral sex ("went down") on your partner(s)? (Use condom/barrier use scale)

H. Ask Female/Gender Unknown Clients who had Male Partners

1a. How many men did you have vaginal sex with?
If 0 , then skip to next section appropriate for the sex of this client. Number cannot exceed total number of male
partners (C3).
1b. How often did you have vaginal sex? (Use frequency scale)
1c. How often did you use a condom? (Use condom/barrier use scale)

ADAI Sound Data Source-3/23/2007

\{Project information\}

http://adai.washington.edu/sounddatasource 


\section{Appendix B}

\section{Risk Behavior Survey (Spanish Version)}

\section{I1. USO DE DROGAS}

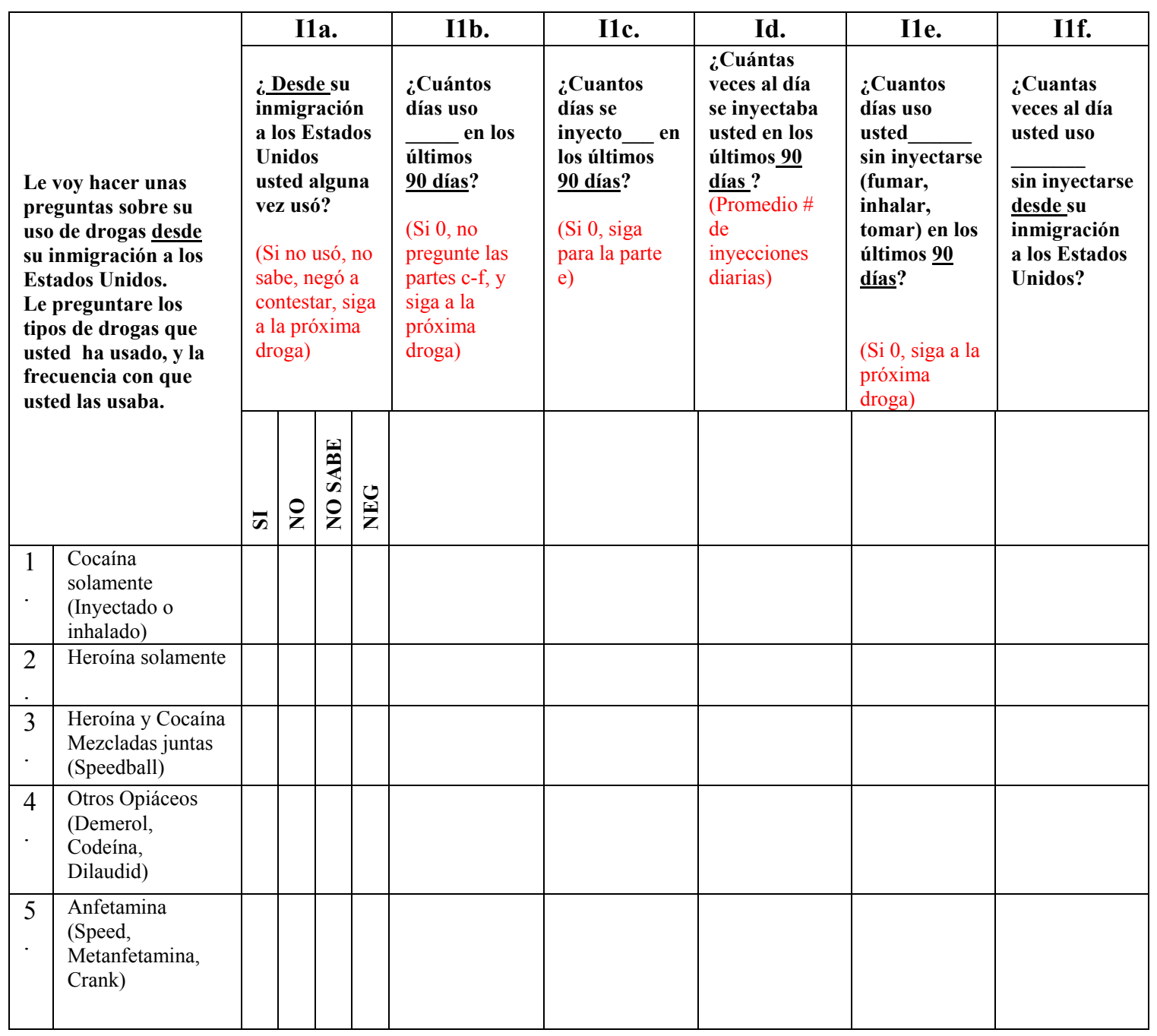

\section{(Si todas las respuestas son "No" $\rightarrow$ I3)}

I2. DROGAS INYECTADAS (si no hubo inyección en los últimos 90 días, siga para la Sección H3)

I2a. ¿En los 90 días cuántas veces (\# de inyecciones) usted se inyectó usando (aguja/jeringuillas) qué usted sabe que fueron usadas por alguien más? (Si ninguno, entre 000 y siga a I2c).

I2b ¿De las veces que usted se inyectó después de otra persona, cuántas veces limpió usted las (aguja/jeringuillas) con cloro de máxima potencia? (El número no puede exceder el número total de veces usados después de que alguien más I2a).

I2c. ¿Cuántas veces en los últimos 90 días usó usted un (cooker/cotton/rinse water) que había sido 
usada por otro inyector?

I2d. ¿Cuántas veces en los 90 días antes preparo drogas con otra persona, y luego compartió la solución de drogas (por el uso de la misma cocina/cuchara o por (front or back loading)?

\section{I3. ACVITIVAD SEXUAL}

Ahora le voy a hacer algunas preguntas sobre sexo. Me refiero aquí a cualquier persona con quien usted tuvo sexo en los últimos $\underline{90 \text { días }}$.

I3a. ¿En los últimos 90 días, con cuántas personas usted tuvo sexo vaginal, oral o anal? SI NINGUNO, ENTRE 000 Y ESTA COMPLETADO ESTA SECCION, IR A SECCION "J" Si>1, ir a I3b

I3b. ¿Cuántos de sus compañeros eran femeninos? número total de gente (I 3a). (Numero no puede exceder el

I3c. ¿Cuántos de sus compañeros eran masculinos? número total de gente (I3a).

(Numero no puede exceder el

I3d. Entrevistador: Codifique el género de la persona: (Marque uno) Hombre $=1$ Mujer $=2$ No Sabe $=9$

Hombre, complete las secciones H.4, H.5, H.6, H.7 \& H.9 Mujer, complete las secciones H.4, H.7, H.8 \& H.9

If Don't Know, ask ALL sex/gender specific questions and allow client to answer as they like

I4. Pregunte a Hombres/Mujeres/Género Desconocido que hayan tenido Parejas Femeninas

I4a ¿Cuántas mujeres le han hecho a usted sexo oral? Si O, siga a la pregunta $\mathrm{H} 4 \mathrm{~d}$. El número no puede exceder el número total de parejas femeninos ( $H$ 3b).

I4b ¿Con que frecuencia le hizo sexo oral su pareja (s) a usted? (TARJETA 15)

I4c. ¿Con qué frecuencia usó usted condones / dental dam, plástico cuando su pareja (s) le hizo sexo oral a usted? (TARJETA 16)

I4d. ¿A Cuántas mujeres le a hecho usted sexo oral? (Si 0, siga a la siguiente sección apropiada para el genero de la persona. El número no puede exceder el número total de parejas femeninas) (I 3b).

I4e. ¿Con qué frecuencia le hizo usted sexo oral a su pareja(s)? (TARJETA 15)

I4f. ¿Con qué frecuencia usó usted condones / dental dam-plásticos cuando usted le hizo sexo oral a su pareja (s)? (TARJETA 16)

\section{I5. Pregunte a Hombres/Género Desconocido que hayan tenido Parejas Femeninas}

I5a. ¿Con cuántas mujeres a tenido usted sexo vaginal? Si 0 , siga a la pregunta $H 5 b$. El número no puede exceder el número total de parejas femeninas (I 3b).

I5b. ¿Con qué frecuencia tuvo usted sexo vaginal? (TARJETA 15)

I5c. ¿Con qué frecuencia usó usted un condón? (TARJETA 16) 
I5d. ¿Con cuántas mujeres a tenido usted sexo anal? (Si 0, siga a la siguiente sección apropiado para el género de la persona. El número no puede exceder el número total de pareja femeninos) (I3b).

I5e. ¿Con qué frecuencia tuvo usted sexo anal? (TARJETA 15)

I5f. ¿Con qué frecuencia usó usted un condón? (TARJETA 16)

I6. Pregunte a Hombres/Género Desconocido que hayan tenido Parejas Masculinos

I6a. ¿Con cuántos hombres tuvo usted sexo anal insertivo? (Si 0, siga a la pregunta I 7a. El número no puede exceder el número total de parejas masculinos) (I 3c).

I6b. ¿Con que frecuencia tuvo usted sexo anal insertivo? (TARJETA 15)

I6c. ¿Con que frecuencia uso usted un condón? (TARJETA 16)

I7. Pregunte a Hombres/Mujeres/Género Desconocido que hayan tenido Parejas Masculinos

I7a. ¿Cuántos hombres le han hecho a usted sexo oral? (Si 0, siga a la pregunta I7d. El número no puede exceder el número total de parejas masculinos) (I 3c).

I7b. ¿Con que frecuencia le hizo sexo oral su pareja (s) a usted? (TARJETA 15)

I7c. ¿Con qué frecuencia uso condones / dental dam, plástico su pareja (s) cuando le hizo sexo oral a usted? (TARJETA 16)

I7d. ¿A Cuántos hombres le ha hecho usted el sexo oral? (Si 0, siga a la siguiente sección apropiado para el género de la persona. El número no puede exceder el número total de parejas masculinos) (I3C)

I7e. ¿Con qué frecuencia le hizo usted el sexo oral a su pareja(s)? (TARJETA 15)

I7f. ¿Con qué frecuencia usó usted condones / dental dam-plásticos cuando usted le hizo sexo oral a su pareja(s)? (TARJETA 16)

\section{I8. Pregunte a Mujeres/Género Desconocido que hayan tenido Parejas Masculinos}

I8a. ¿Con cuántos hombres ha tenido usted sexo vaginal? (Si 0 , siga a la siguiente sección apropiada para el género de la persona. El número no puede exceder el número total de parejas masculinos) (I3c).

I8b. ¿Con que frecuencia tuvo usted sexo vaginal? (TARJETA 15)

I8c. ¿Con que frecuencia uso usted un condón? (TARJETA 16)

I9. Pregunte a Hombres/Mujeres/Género Desconocido que hayan tenido Parejas Masculinos

19.1. ¿Con cuántos hombres tuvo usted sexo anal receptivo? (Si 0, siga a la sección I) El número no puede exceder el número total de parejas masculinos) (I 3c).

19.2. ¿Con que frecuencia tuvo usted sexo anal receptivo? (TARJETA 15)

19.3. ¿Con que frecuencia uso usted un condón? (TARJETA 16) 


\section{The Alcohol Use Disorders Identification Test}

The next questions will ask about your use of alcohol in the last 12 months. These questions refer to your use of alcohol. Remember to please answer honestly and to the best of your ability. In the last 12 months...

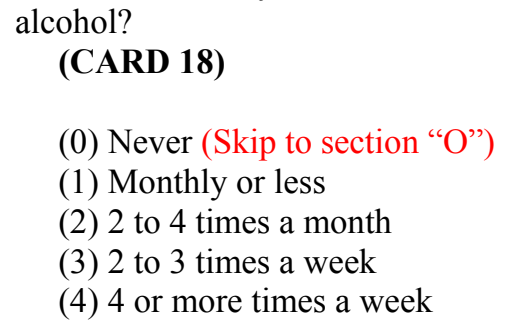
alcohol?

\section{(CARD 18)}

(0) Never (Skip to section "O")

(1) Monthly or less

(2) 2 to 4 times a month

(3) 2 to 3 times a week

(4) 4 or more times a week

1. How often do you have a drink containing

3. How often do you have 5 or more a drinks on one occasion? (CARD 20)
(0) Never
(1) Less than monthly
(2) Monthly
(3) Weekly
(4) Daily or almost daily

2. How many drinks containing alcohol do you have on a typical day when you are drinking? (CARD 19)
(0) 1 or 2
(1) 3 or 4
(2) 5 or 6
(3) 7,8 or 9
(4) 10 or more

4. How often during the last year have you found that you were not able to stop drinking once you had started?

(CARD 20)
(0) Never
(1) Less than monthly
(2) Monthly
(3) Weekly
(4) Daily or almost daily

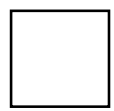

5. How often during the last year have you failed to do what was normally expected from you because of your drinking? (CARD 20)
(0) Never
(1) Less than monthly
(2) Monthly
(3) Weekly
(4) Daily or almost daily

6. How often during the last year have you needed a first drink in the morning to get yourself going after a heavy drinking session? (CARD 20)
(0) Never
(1) Less than monthly
(2) Monthly
(3) Weekly
(4) Daily or almost daily

7. How often during the last year have you had feelings of guilt or remorse after drinking? (CARD 20)

(0) Never

(1) Less than monthly

(2) Monthly

(3) Weekly

(4) Daily or almost daily
8. How often during the last year have you been unable to remember what happened the night before because you had been drinking ?

(CARD 20)
(0) Never
(1) Less than monthly
(2) Monthly
(3) Weekly
(4) Daily or almost daily 
9. Have you or someone else been injured as a result of your drinking? (CARD 21)

(0) No

(2) Yes, but not in the last year

(4) Yes, during the last year
10. Has a relative or friend or a doctor or other health worked been concerned about your drinking or suggested you cut down? (CARD 21)

(0) No

(2) Yes, but not in the last year

(4) Yes, during the last year 


\section{The Alcohol Use Disorders Identification Test (Spanish Version)}

\section{Las siguientes preguntas son sobre su uso de alcohol en los últimos 12 meses. Estas preguntas se refieren a su uso de alcohol. Acuérdese de por favor responder lo más honesto posible y a su mejor capacidad. En los últimos 12 meses.}

\begin{tabular}{l|l|}
$\begin{array}{l}\text { 1. ¿Con que frecuencia ingiere bebidas alcohólicas? } \\
\text { (TARJETA 18) }\end{array}$ & $\begin{array}{l}\text { 2. ¿Cuántas bebidas alcohólicas ingiere en un día } \\
\text { típico cuando está tomando? (TARJETA 19) }\end{array}$ \\
& (0) 1 o 2 \\
(0) Nunca (Siga a sección "O") & (1) 3 o 4 \\
(1) Mensualmente o Menos & (2) 5 o 6 \\
(2) 2 a 4 veces por mes & (3) 7,8 or 9 \\
(3) 2 a 3 veces por semana & (4) 10 o more \\
(4) 4 veces o mas por semana &
\end{tabular}

3. ¿Con que frecuencia toma más de cinco cervezas o tragos en la misma ocasión? (TARJETA 20)

(0) Nunca

(1) Menos de una vez al mes

(2) Mensualmente

(3) Semanalmente

(4) Diario o casi diario
4. ¿Le ocurrió, durante el último año, que no pudo parar de beber una vez que había empezado?

(TARJETA 20)

(0) Nunca

(1) Menos de una vez al mes

(2) Mensualmente

(3) Semanalmente

(4) Diario o casi diario

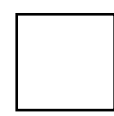

5. ¿Que tan frecuentemente, durante el último año, dejo de hacer algo que debería haber hecho por beber?

(TARJETA 20)

6. ¿Que tan frecuentemente, durante el último año, necesito beber un trago a la mañana siguiente después de haber bebido en exceso? (TARJETA 20)

(0) Nunca

(1) Menos de una vez al mes

(0) Nunca

(2) Mensualmente

(3) Semanalmente

(4) Diario o casi diario

(1) Menos de una vez al mes

(2) Mensualmente

(3) Semanalmente

(4) Diario o casi diario

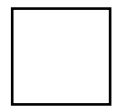

7. ¿Que tan frecuentemente, durante el último año, se sintió culpable o tuvo remordimientos por haber bebido?

(TARJETA 20)

8. ¿Que tan frecuentemente, durante el último año, olvido algo de lo que había pasado la noche anterior debido a que estuvo bebiendo?

(TARJETA 20)

(0) Nunca

(1) Menos de una vez al mes

(0) Nunca

(2) Mensualmente

(1) Menos de una vez al mes

(3) Semanalmente

(4) Diario o casi diario

(2) Mensualmente

(3) Semanalmente

(4) Diario o casi diario 
9. ¿Se ha lastimado o alguien a resultado lastimado como consecuencia de su consumo de bebidas alcohólicas?

(0) No

(2) $\mathrm{Si}$, pero no en el último año

(4) $\mathrm{Si}$, en el último año
10. ¿Algún amigo, familiar, o doctor se ha preocupado por la forma en que usted bebe o le ha sugerido que disminuya el consumo?

(0) No

(2) $\mathrm{Si}$, pero no en el último año

(4) $\mathrm{Si}$, en el último año

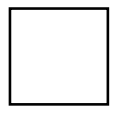


Appendix E

Timeline Followback

\section{TIMELINE FOLLOWBACK METHOD (ALCOHOL)}

The next set of questions will ask about your use of alcohol in the last 90 days. We will be looking at a calendar together to help you recall this time period. Filling out the calendar is not hard. Try to be as accurate as possible. We recognize that you won't have perfect recall. If you are unsure of how many drinks you had on any particular day, your best guess is ok. We will be putting a number in each of the days of the calendar. If you did not drink on any particular day we will mark a " 0 ." If you did drink alcohol we will mark the number of drinks that you had on that day. The idea is to put a number in for each day on the calendar. If you have regular drinking patterns you may use these to help you recall your drinking. For example, if you have a daily or weekend/weekday drinking pattern, or drink on Wednesdays after playing sports, etc. Remember, the time period we are talking about in the last 3 months.

(INTERVIEWER: Please fill out months accordingly on the calendar.)

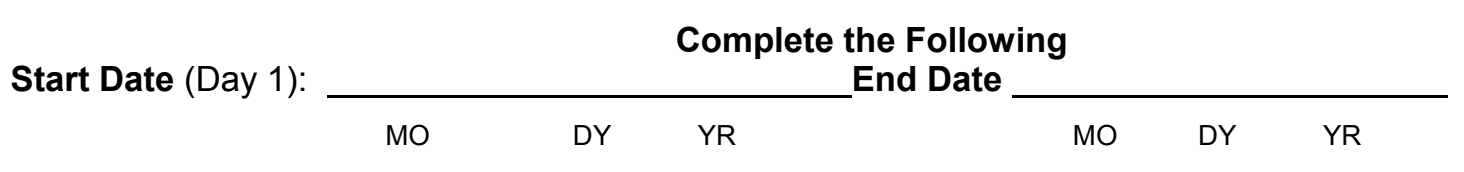

\section{TIMELINE FOLLOWBACK METHOD (DRUGS)}

The next set of questions asks about the drugs you have used on your own in the last 90 days. By "on your own" mean any drugs that you have used without a prescription from a doctor, or for longer periods or larger quantities than prescribed. This includes and is not limited to marijuana, sedatives (valium, xanax,), stimulants (cocaine, amphetamines, crystal meth), heroin, opioids (morphine, methadone, codeine), hallucinogens (LSD, mushrooms, etc.) We will, again, be looking at a calendar together to help you recall this time period. Please try to be as accurate and honest as possible. On the days you did not use drugs we will mark a "0." On the days you did use drugs we will make a check mark. Remember, the time period we are talking about in the last 3 months. 


\section{Appendix F}

Timeline Followback (Spanish Version)

\section{Metodo Timeline Followback (Alcohol)}

Las siguientes preguntas son sobre su uso de alcohol en los 90 días. Miraremos un calendario junto para ayudarle a recordar este período de tiempo. Llenar el calendario no es difícil. Trate de ser lo más exacto posible. Reconocemos que usted no recordara con exactitud. Si usted no está seguro de cuantas bebidas tomo durante algún día en particular, su mejor estimación está bien. Marcaremos un número en cada uno de los días del calendario. Si usted no bebió un día en particular marcaremos "un 0". Si usted si bebió marcaremos el número de bebidas que usted tomo durante ese día. La idea es marcar un número en cada día del calendario. Si usted tiene un patrón regular de beber puede usarlo para ayudarle a recordar cuanto ha bebido. Por ejemplo, si usted bebe diariamente o en los fines de semana/día laborables, o si bebe los miércoles después de jugar deportes, etc. Recuerde, el período de tiempo del que hablamos serán los últimos $\mathbf{3}$ meses. (ENTREVISTOR: Por favor llene los meses apropiados en el calendario)

Complete lo Siguiente

Día de Empiezo (Día 1): Día de Termino

MO / DY / YR MO / DY / YR

\section{Metodo Timeline Followback (Drogas)}

Las siguientes preguntas son sobre las drogas que usted ha usado por su propia decisión en los últimos 90 días. Por "su propia decisión" queremos decir cualquier medicina o droga que usted ha usado sin una prescripción de un doctor, o durante períodos más largos o cantidades más grandes que prescribidlo. Esto incluye pero no es limitado a la marihuana, sedantes (valium, xanax,), estimulantes (cocaína, anfetaminas, cristal meth), heroína, opioids (morfina, metadona, codeína), alucinógenos (LSD, setas, etc.)

Miraremos, otra vez, el calendario junto para ayudarle a recordar este período de tiempo. Por favor trate de ser lo más exacto y honesto posible. Durante los días en que usted no uso drogas marcaremos "un 0". Durante los días en que usted si uso drogas haremos una marca de verificación. Recuerde, el período de tiempo del que hablamos serán los últimos 3 meses. 


\section{Appendix G}

Hispanic Stress Inventory-Immigrant Version

The next set of questions will talk about how things have been for you since you have been living in the United States. For the following statements please tell me whether any of these situations have occurred to you during the time you have been living in the United States. Then if it did occur to you, indicate how worried or tense the situation was to you. Remember there is no right or wrong answer so try and be as honest as you can.

J1. Because I don't know enough English, it has been difficult for me to interact with others.

\begin{tabular}{|c|c|c|c|c|} 
Yes [ ] No [ ] & Moderately \\
\hline $\begin{array}{c}\text { Not at all } \\
\text { worried/tense }\end{array}$ & $\begin{array}{c}\text { A little } \\
\text { worried/tense }\end{array}$ & $\begin{array}{c}\text { Very } \\
\text { worried/tense }\end{array}$ & $\begin{array}{c}\text { Extremely } \\
\text { worried/tense } \\
\text { worried/tense }\end{array}$ \\
\hline 1 & 2 & 3 & 4 & 5 \\
\hline
\end{tabular}

J2. I have felt pressured to learn English.

\begin{tabular}{|c|c|c|c|c|}
\multicolumn{4}{c}{ Yes [ ] } & No [ ] N/A [ ] \\
\hline $\begin{array}{c}\text { Not at all } \\
\text { worried/tense }\end{array}$ & $\begin{array}{c}\text { A little } \\
\text { worried/tense }\end{array}$ & $\begin{array}{c}\text { Moderately } \\
\text { worried/tense }\end{array}$ & $\begin{array}{c}\text { Very } \\
\text { worried/tense }\end{array}$ & $\begin{array}{c}\text { Extremely } \\
\text { worried/tense }\end{array}$ \\
\hline 1 & 2 & 3 & 4 & 5 \\
\hline
\end{tabular}

J3. Because I am Latino, I have had difficulty finding the type of work I want.

Yes [ ] No [

\begin{tabular}{|c|c|c|c|c|}
\hline $\begin{array}{c}\text { Not at all } \\
\text { worried/tense }\end{array}$ & $\begin{array}{c}\text { A little } \\
\text { worried/tense }\end{array}$ & $\begin{array}{c}\text { Moderately } \\
\text { worried/tense }\end{array}$ & $\begin{array}{c}\text { Very } \\
\text { worried/tense }\end{array}$ & $\begin{array}{c}\text { Extremely } \\
\text { worried/tense }\end{array}$ \\
\hline 1 & 2 & 3 & 4 & 5 \\
\hline
\end{tabular}

J4. I have thought if I went to a social or government agency I would be deported.

\begin{tabular}{|c|c|c|c|c|}
\multicolumn{1}{c|}{ Yes [ ] No [ ] at all } \\
\begin{tabular}{|c|c|c|c|} 
Not little \\
worried/tense
\end{tabular} & $\begin{array}{c}\text { Moderately } \\
\text { worried/tense }\end{array}$ & $\begin{array}{c}\text { Very } \\
\text { worried/tense } \\
\text { worried/tense }\end{array}$ & $\begin{array}{c}\text { Extremely } \\
\text { worried/tense }\end{array}$ \\
\hline 1 & 2 & 3 & 4 & 5 \\
\hline
\end{tabular}

J5. Because of my poor English people have treated me badly.

\begin{tabular}{|c|c|c|c|c|}
\multicolumn{1}{|c}{ Yes [ ] No [ ] } \\
\begin{tabular}{|c|c|c|} 
Not at all \\
worried/tense
\end{tabular} & $\begin{array}{c}\text { A little } \\
\text { worried/tense }\end{array}$ & $\begin{array}{c}\text { Moderately } \\
\text { worried/tense }\end{array}$ & $\begin{array}{c}\text { Very } \\
\text { worried/tense }\end{array}$ & $\begin{array}{c}\text { Extremely } \\
\text { worried/tense }\end{array}$ \\
\hline 1 & 2 & 3 & 4 & 5 \\
\hline
\end{tabular}


J6. Because my poor English, it has been difficult for me to deal with day to day situations.

\begin{tabular}{|c|c|c|c|c|}
\hline \multicolumn{5}{|c|}{ Yes [ ] No [ ] } \\
\hline $\begin{array}{c}\text { Not at all } \\
\text { worried/tense }\end{array}$ & $\begin{array}{c}\text { A little } \\
\text { worried/tense }\end{array}$ & $\begin{array}{c}\text { Moderately } \\
\text { worried/tense }\end{array}$ & $\begin{array}{c}\text { Very } \\
\text { worried/tense }\end{array}$ & $\begin{array}{c}\text { Extremely } \\
\text { worried/tense }\end{array}$ \\
\hline 1 & 2 & 3 & 4 & 5 \\
\hline
\end{tabular}

J7. I feared the consequences of deportation.

\begin{tabular}{|c|c|c|c|c|}
\multicolumn{1}{c|}{ Yes [ ] Not at all } & $\begin{array}{c}\text { A little } \\
\text { worried/tense }\end{array}$ & $\begin{array}{c}\text { Moderately } \\
\text { worried/tense }\end{array}$ & $\begin{array}{c}\text { Very } \\
\text { worried/tense }\end{array}$ & $\begin{array}{c}\text { Extremely } \\
\text { worried/tense }\end{array}$ \\
\hline worried/tense & 2 & 3 & 4 & 5 \\
\hline 1 & 2 & & 4 & 5
\end{tabular}

J8.I have avoided immigration officials.

Yes [ ] No [ ]
\begin{tabular}{|c|c|c|c|c|}
\hline $\begin{array}{c}\text { Not at all } \\
\text { worried/tense }\end{array}$ & $\begin{array}{c}\text { A little } \\
\text { worried/tense }\end{array}$ & $\begin{array}{c}\text { Moderately } \\
\text { worried/tense }\end{array}$ & $\begin{array}{c}\text { Very } \\
\text { worried/tense }\end{array}$ & $\begin{array}{c}\text { Extremely } \\
\text { worried/tense }\end{array}$ \\
\hline 1 & 2 & 3 & 4 & 5 \\
\hline
\end{tabular}

J9. Due to problems understanding English, I have had difficulties in school.

\begin{tabular}{|c|c|c|c|c|}
\multicolumn{2}{c|}{ Yes [ ] } & No [ ] N/A [ ] & Extremely \\
\hline $\begin{array}{c}\text { Not at all } \\
\text { worried/tense }\end{array}$ & $\begin{array}{c}\text { A little } \\
\text { worried/tense }\end{array}$ & $\begin{array}{c}\text { Moderately } \\
\text { worried/tense }\end{array}$ & $\begin{array}{c}\text { Very } \\
\text { worried/tense }\end{array}$ & $\begin{array}{c}\text { Extried/tense } \\
\text { worried }\end{array}$ \\
\hline 1 & 2 & 3 & 4 & 5 \\
\hline
\end{tabular}

J10. I have had difficulties finding legal services.

\begin{tabular}{|c|c|c|c|c|}
\multicolumn{2}{c|}{ Yes [ ] } & No [ ] N/A [ ] & [ \\
\hline $\begin{array}{c}\text { Not at all } \\
\text { worried/tense }\end{array}$ & $\begin{array}{c}\text { A little } \\
\text { worried/tense }\end{array}$ & $\begin{array}{c}\text { Moderately } \\
\text { worried/tense }\end{array}$ & $\begin{array}{c}\text { Very } \\
\text { worried/tense }\end{array}$ & $\begin{array}{c}\text { Extremely } \\
\text { worried/tense }\end{array}$ \\
\hline 1 & 2 & 3 & 4 & 5 \\
\hline
\end{tabular}

J11. I felt guilty about leaving my family and friends in my home country.

\begin{tabular}{|c|c|c|c|c|}
\multicolumn{1}{c}{ Yes [ ] } & No [ ] N/A [ ] & Very \\
\hline $\begin{array}{c}\text { Not at all } \\
\text { worried/tense }\end{array}$ & $\begin{array}{c}\text { A little } \\
\text { worried/tense }\end{array}$ & $\begin{array}{c}\text { Moderately } \\
\text { worried/tense }\end{array}$ & $\begin{array}{c}\text { Extremely } \\
\text { worried/tense } \\
\text { worried/tense }\end{array}$ \\
\hline 1 & 2 & 3 & 4 & 5 \\
\hline
\end{tabular}

J12. My legal status has limited my contact with family and friends. Yes [ ] No [ ]

\begin{tabular}{|c|c|c|c|c|}
\hline $\begin{array}{c}\text { Not at all } \\
\text { worried/tense }\end{array}$ & $\begin{array}{c}\text { A little } \\
\text { worried/tense }\end{array}$ & $\begin{array}{c}\text { Moderately } \\
\text { worried/tense }\end{array}$ & $\begin{array}{c}\text { Very } \\
\text { worried/tense }\end{array}$ & $\begin{array}{c}\text { Extremely } \\
\text { worried/tense }\end{array}$ \\
\hline 1 & 2 & 3 & 4 & 5 \\
\hline
\end{tabular}


J13. I have felt that I will never regain the status and respect I had in my home country.

\begin{tabular}{|c|c|c|c|c|}
\hline $\begin{array}{c}\text { Not at all } \\
\text { worried/tense }\end{array}$ & $\begin{array}{c}\text { A little } \\
\text { worried/tense }\end{array}$ & $\begin{array}{c}\text { Moderately } \\
\text { worried/tense }\end{array}$ & $\begin{array}{c}\text { Very } \\
\text { worried/tense }\end{array}$ & $\begin{array}{c}\text { Extremely } \\
\text { worried/tense }\end{array}$ \\
\hline 1 & 2 & 3 & 4 & 5 \\
\hline
\end{tabular}

J14. I have felt unaccepted by others due to my Latino culture.

\begin{tabular}{|c|c|c|c|c|}
\hline $\begin{array}{c}\text { Not at all } \\
\text { worried/tense }\end{array}$ & $\begin{array}{c}\text { A little } \\
\text { worried/tense }\end{array}$ & $\begin{array}{c}\text { Moderately } \\
\text { worried/tense }\end{array}$ & $\begin{array}{c}\text { Very } \\
\text { worried/tense }\end{array}$ & $\begin{array}{c}\text { Extremely } \\
\text { worried/tense }\end{array}$ \\
\hline 1 & 2 & 3 & 4 & 5 \\
\hline
\end{tabular}

J15. I have been discriminated against.

\begin{tabular}{|c|c|c|c|c|}
\hline $\begin{array}{c}\text { Not at all } \\
\text { worried/tense }\end{array}$ & $\begin{array}{c}\text { A little } \\
\text { worried/tense }\end{array}$ & $\begin{array}{c}\text { Moderately } \\
\text { worried/tense }\end{array}$ & $\begin{array}{c}\text { Very } \\
\text { worried/tense }\end{array}$ & $\begin{array}{c}\text { Extremely } \\
\text { worried/tense }\end{array}$ \\
\hline 1 & 2 & 3 & 4 & 5 \\
\hline
\end{tabular}

J16. I've been questioned about my legal status

\begin{tabular}{|c|c|c|c|c|}
\multicolumn{1}{c|}{ Yes [ ] } & No [ ] \\
\begin{tabular}{|c|c|c|c|} 
Not at all \\
worried/tense
\end{tabular} & $\begin{array}{c}\text { A little } \\
\text { worried/tense }\end{array}$ & $\begin{array}{c}\text { Moderately } \\
\text { worried/tense }\end{array}$ & $\begin{array}{c}\text { Very } \\
\text { worried/tense }\end{array}$ & $\begin{array}{c}\text { Extremely } \\
\text { worried/tense }\end{array}$ \\
\hline 1 & 2 & 3 & 4 & 5 \\
\hline
\end{tabular}

J17. I have not been able to forget the war related deaths, which have happened to friend and family members.

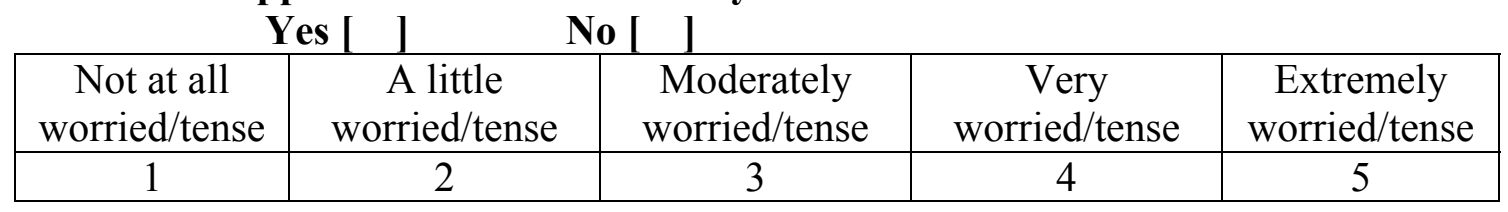

J18. I have not been able to forget the last few months in my home country.

\begin{tabular}{|c|c|c|c|c|}
\multicolumn{1}{c}{ Yos [ ] at all } & $\begin{array}{c}\text { A little } \\
\text { worried/tense }\end{array}$ & $\begin{array}{c}\text { Moderately } \\
\text { worried/tense }\end{array}$ & $\begin{array}{c}\text { Very } \\
\text { worried/tense }\end{array}$ & $\begin{array}{c}\text { Extremely } \\
\text { worried/tense }\end{array}$ \\
\hline 1 & 2 & 3 & 4 & 5 \\
\hline
\end{tabular}




\section{Appendix $\mathrm{H}$}

\section{Hispanic Stress Inventory-Immigrant Version (Spanish Version)}

Las siguiente preguntas serán sobre como las cosas le han ido a usted en los últimos 12 meses des de que ha estado viviendo en los Estados Unidos. Para las siguientes frases por favor dígame si cualquiera de estas situaciones le ha ocurrido durante los últimos 12 meses. Entonces si esto si le ocurrió, indique cuanta preocupación o tensión esta situación le ha hecho sentir. Recuerde que no hay repuestas correctas o incorrectas así que por favor trate de ser lo más honesto posible.

K1. Por no saber suficiente Ingles ha sido difícil para mí interactuar con otros.

\begin{tabular}{|c|c|c|c|c|}
\multicolumn{1}{c|}{ Si [ ] No [ ] } \\
\hline $\begin{array}{c}\text { Nada } \\
\text { Preocupado/Tenso }\end{array}$ & $\begin{array}{c}\text { Un Poco } \\
\text { Preocupado/Tenso }\end{array}$ & $\begin{array}{c}\text { Moderadamente } \\
\text { Preocupado/Tenso }\end{array}$ & $\begin{array}{c}\text { Muy } \\
\text { Preocupado/Tenso }\end{array}$ & $\begin{array}{c}\text { Extremadamente } \\
\text { Preocupado/Tenso }\end{array}$ \\
\hline 1 & 2 & 3 & 4 & 5 \\
\hline
\end{tabular}

K2. Me he sentido presionado/a para aprender Ingles.

\begin{tabular}{|c|c|c|c|c|}
\multicolumn{1}{c}{ Si [ ] No [ ] } & N/A [ ] & Muy & $\begin{array}{c}\text { Extremadamente } \\
\text { Preocupado/Tenso }\end{array}$ \\
\hline Nada & Un Poco & Moderadamente & Preocupado/Tenso & Preocupado/Tenso \\
\hline 1 & 2 & 3 & 4 & 5 \\
\hline
\end{tabular}

K3. Debido a que soy Latino/a he tenido dificultad para encontrar el tipo de trabajo que

\begin{tabular}{|c|c|c|c|c|}
\hline quiero. & Si [ ] & ] & & \\
\hline $\begin{array}{c}\text { Nada } \\
\text { Preocupado/Tenso }\end{array}$ & $\begin{array}{c}\text { Un Poco } \\
\text { Preocupado/Tenso }\end{array}$ & $\begin{array}{c}\text { Moderadamente } \\
\text { Preocupado/Tenso }\end{array}$ & $\begin{array}{c}\text { Muy } \\
\text { Preocupado/Tenso }\end{array}$ & $\begin{array}{l}\text { Extremadamente } \\
\text { Preocupado/Tenso }\end{array}$ \\
\hline 1 & 2 & 3 & 4 & 5 \\
\hline
\end{tabular}

K4. He pensado que si fuera a una agencia de servicios social o del gobierno, sería deportado/a

\begin{tabular}{|c|c|c|c|c|}
\multicolumn{1}{c|}{ Si [ ] No [ ] } & N/A [ ] & Muy & $\begin{array}{c}\text { Extremadamente } \\
\text { Preocupado/Tenso }\end{array}$ \\
\hline Nada & $\begin{array}{c}\text { Un Poco } \\
\text { Preocupado/Tenso }\end{array}$ & $\begin{array}{c}\text { Moderadamente } \\
\text { Preocupado/Tenso }\end{array}$ & $\begin{array}{c}\text { Preocupado/Tenso } \\
\text { Preocupado/Tenso }\end{array}$ & 3 \\
\hline 1 & 2 & 3 & 4 & 5 \\
\hline
\end{tabular}

K5. Debido a mi mal Ingles, la gente me ha tratado mal.

\begin{tabular}{|c|c|c|c|c|}
\multicolumn{1}{c|}{ Si [ ] No [ ] } & \multicolumn{4}{c|}{} \\
\hline Nada & Un Poco & Moderadamente & Muy & Extremadamente \\
Preocupado/Tenso & Preocupado/Tenso & Preocupado/Tenso & Preocupado/Tenso & Preocupado/Tenso \\
\hline 1 & 2 & 3 & 4 & 5 \\
\hline
\end{tabular}


K6. Por no saber suficiente Ingles ha sido difícil el enfrentar situaciones diarias como ir de compras o tomar el auto bus.

\begin{tabular}{|c|c|c|c|c|}
\multicolumn{1}{c|}{ Si [ ] No [ ] } & \multicolumn{1}{c|}{} \\
\hline $\begin{array}{c}\text { Nada } \\
\text { Preocupado/Tenso }\end{array}$ & $\begin{array}{c}\text { Un Poco } \\
\text { Preocupado/Tenso }\end{array}$ & $\begin{array}{c}\text { Moderadamente } \\
\text { Preocupado/Tenso }\end{array}$ & $\begin{array}{c}\text { Muy } \\
\text { Preocupado/Tenso }\end{array}$ & $\begin{array}{c}\text { Extremadamente } \\
\text { Preocupado/Tenso }\end{array}$ \\
\hline 1 & 2 & 3 & 4 & 5 \\
\hline
\end{tabular}

K7. He tenido miedo a las consecuencias de la deportación.

\begin{tabular}{|c|c|c|c|c|}
\hline $\begin{array}{c}\text { Si } \\
\text { Nada } \\
\text { Preocupado/Tenso }\end{array}$ & $\begin{array}{c}\text { No [ ] } \\
\text { Un Poco } \\
\text { Preocupado/Tenso }\end{array}$ & $\begin{array}{c}\text { N/A [ ] } \\
\text { Moderadamente } \\
\text { Preocupado/Tenso }\end{array}$ & $\begin{array}{c}\text { Muy } \\
\text { Preocupado/Tenso }\end{array}$ & $\begin{array}{l}\text { Extremadamente } \\
\text { Preocupado/Tenso }\end{array}$ \\
\hline 1 & 2 & 3 & 4 & 5 \\
\hline
\end{tabular}

K8. He evitado enfrentamientos con agentes de inmigración.

\begin{tabular}{|c|c|c|c|c|}
\multicolumn{1}{c|}{ Si [ ] } & No [ ] & \multicolumn{1}{c|}{} \\
\hline Nada & Un Poco & Moderadamente & Muy & Extremadamente \\
Preocupado/Tenso & Preocupado/Tenso & Preocupado/Tenso & Preocupado/Tenso & Preocupadonso \\
\hline 1 & 2 & 3 & 4 & 5 \\
\hline
\end{tabular}

K9. Debido a problemas con el Ingles, he tenido dificultades en la escuela o Universidad.

\begin{tabular}{|c|c|c|c|c|}
\multicolumn{1}{c|}{ Si [ ] No [ ] } & N/A [ ] & \multicolumn{1}{c|}{} \\
\hline Nada & $\begin{array}{c}\text { Un Poco } \\
\text { Preocupado/Tenso }\end{array}$ & $\begin{array}{c}\text { Moderadamente } \\
\text { Preocupado/Tenso }\end{array}$ & $\begin{array}{c}\text { Muy } \\
\text { Preocupado/Tenso }\end{array}$ & $\begin{array}{c}\text { Extremadamente } \\
\text { Preocupado/Tenso }\end{array}$ \\
\hline 1 & 2 & 3 & 4 & 5 \\
\hline
\end{tabular}

K10. He tenido dificultad para encontrar ayuda/servicios legales.

\begin{tabular}{|c|c|c|c|c|}
\hline $\begin{array}{c}\text { Nada } \\
\text { Preocupado/Tenso }\end{array}$ & $\begin{array}{c}\text { Un Poco } \\
\text { Preocupado/Tenso }\end{array}$ & $\begin{array}{c}\text { Moderadamente } \\
\text { Preocupado/Tenso }\end{array}$ & $\begin{array}{c}\text { Muy } \\
\text { Preocupado/Tenso }\end{array}$ & $\begin{array}{l}\text { Extremadamente } \\
\text { Preocupado/Tenso }\end{array}$ \\
\hline 1 & 2 & 3 & 4 & 5 \\
\hline
\end{tabular}

K11. Me he sentido culpable por haber dejado a mi familia y a mis amigo/a en mi país.

\begin{tabular}{|c|c|c|c|c|}
\multicolumn{1}{|c}{ Si [ ] No [ ] } & N/A [ ] & \multicolumn{2}{c|}{} \\
\hline $\begin{array}{c}\text { Nada } \\
\text { Preocupado/Tenso }\end{array}$ & $\begin{array}{c}\text { Un Poco } \\
\text { Preocupado/Tenso }\end{array}$ & $\begin{array}{c}\text { Moderadamente } \\
\text { Preocupado/Tenso }\end{array}$ & $\begin{array}{c}\text { Muy } \\
\text { Preocupado/Tenso }\end{array}$ & $\begin{array}{c}\text { Extremadamente } \\
\text { Preocupado/Tenso }\end{array}$ \\
\hline 1 & 2 & 3 & 4 & 5 \\
\hline
\end{tabular}

K12. Mi situación legal en este país ha limitado mi contacto con mi familia y amigos.

\begin{tabular}{|c|c|c|c|c|}
\multicolumn{2}{c|}{ Si [ ] } & No [ ] & \multicolumn{3}{c|}{} \\
\hline Nada & Un Poco & Moderadamente & Muy & Extremadamente \\
Preocupado/Tenso & Preocupado/Tenso & Preocupado/Tenso & Preocupado/Tenso & Preocupado/Tenso \\
\hline 1 & 2 & 3 & 4 & 5 \\
\hline
\end{tabular}


K13. He sentido que en este país nunca podré recuperar la posición y el respeto que tenía en mí país de origen.

\begin{tabular}{|c|c|c|c|c|}
\multicolumn{1}{c|}{ Si [ ] No [ ] } & \multicolumn{1}{c|}{} \\
\hline $\begin{array}{c}\text { Nada } \\
\text { Preocupado/Tenso }\end{array}$ & $\begin{array}{c}\text { Un Poco } \\
\text { Preocupado/Tenso }\end{array}$ & $\begin{array}{c}\text { Moderadamente } \\
\text { Preocupado/Tenso }\end{array}$ & $\begin{array}{c}\text { Muy } \\
\text { Preocupado/Tenso }\end{array}$ & $\begin{array}{c}\text { Extremadamente } \\
\text { Preocupado/Tenso }\end{array}$ \\
\hline 1 & 2 & 3 & 4 & 5 \\
\hline
\end{tabular}

K14. No me he sentido aceptado/a por otros debido a mi cultura Latina

\begin{tabular}{|c|c|c|c|c|}
\multicolumn{1}{c|}{ Si [ ] No [ ] } & \multicolumn{1}{c|}{} \\
\hline $\begin{array}{c}\text { Nada } \\
\text { Preocupado/Tenso }\end{array}$ & $\begin{array}{c}\text { Un Poco } \\
\text { Preocupado/Tenso }\end{array}$ & $\begin{array}{c}\text { Moderadamente } \\
\text { Preocupado/Tenso }\end{array}$ & $\begin{array}{c}\text { Muy } \\
\text { Preocupado/Tenso }\end{array}$ & $\begin{array}{c}\text { Extremadamente } \\
\text { Preocupado/Tenso }\end{array}$ \\
\hline 1 & 2 & 3 & 4 & 5 \\
\hline
\end{tabular}

K15. Se me ha discriminado.

\begin{tabular}{|c|c|c|c|c|}
\multicolumn{1}{c|}{ Si [ ] No [ ] } & \multicolumn{1}{l|}{} \\
\hline $\begin{array}{c}\text { Nada } \\
\text { Preocupado/Tenso }\end{array}$ & $\begin{array}{c}\text { Un Poco } \\
\text { Preocupado/Tenso }\end{array}$ & $\begin{array}{c}\text { Moderadamente } \\
\text { Preocupado/Tenso }\end{array}$ & $\begin{array}{c}\text { Muy } \\
\text { Preocupado/Tenso }\end{array}$ & $\begin{array}{c}\text { Extremadamente } \\
\text { Preocupado/Tenso }\end{array}$ \\
\hline 1 & 2 & 3 & 4 & 5 \\
\hline
\end{tabular}

K16. Se me ha preguntado sobre mi situación legal en este país.

\begin{tabular}{|c|c|c|c|c|}
\multicolumn{1}{c|}{ Si [ ] } & No [ ] \\
\hline $\begin{array}{c}\text { Nada } \\
\text { Preocupado/Tenso }\end{array}$ & $\begin{array}{c}\text { Un Poco } \\
\text { Preocupado/Tenso }\end{array}$ & $\begin{array}{c}\text { Moderadamente } \\
\text { Preocupado/Tenso }\end{array}$ & $\begin{array}{c}\text { Muy } \\
\text { Preocupado/Tenso }\end{array}$ & $\begin{array}{c}\text { Extremadamente } \\
\text { Preocupado/Tenso }\end{array}$ \\
\hline 1 & 2 & 3 & 4 & 5 \\
\hline
\end{tabular}

K17. No he podido olvidar la muerte de familiares o amigos debido a violencia en mi país?

\begin{tabular}{|c|c|c|c|c|}
\multicolumn{1}{c|}{ Si [ ] No N ] } & \multicolumn{2}{c|}{ N/A [ ] } \\
\hline Nada & $\begin{array}{c}\text { Un Poco } \\
\text { Preocupado/Tenso }\end{array}$ & $\begin{array}{c}\text { Moderadamente } \\
\text { Preocupado/Tenso }\end{array}$ & $\begin{array}{c}\text { Muy } \\
\text { Preocupado/Tenso }\end{array}$ & $\begin{array}{c}\text { Extremadamente } \\
\text { Preocupado/Tenso }\end{array}$ \\
\hline 1 & 2 & 3 & 4 & 5 \\
\hline
\end{tabular}

K18. No he podido olvidar los últimos meses en mi país de origen.

\begin{tabular}{|c|c|c|c|c|}
\hline $\begin{array}{c}\text { Nada } \\
\text { Preocupado/Tenso }\end{array}$ & $\begin{array}{c}\text { Un Poco } \\
\text { Preocupado/Tenso }\end{array}$ & $\begin{array}{c}\text { Moderadamente } \\
\text { Preocupado/Tenso }\end{array}$ & $\begin{array}{c}\text { Muy } \\
\text { Preocupado/Tenso }\end{array}$ & $\begin{array}{c}\text { Extremadamente } \\
\text { Preocupado/Tenso }\end{array}$ \\
\hline 1 & 2 & 3 & 4 & 5 \\
\hline
\end{tabular}


Appendix I

\section{Ways of Religious Coping Scale (WORSC)}

The following questions relate to how you handled stressful situations in your life since you have been in the United States. A "stressful" situation is any situation that is difficult or troubling for you, either because you feel distressed about what is happening or because you have to use considerable effort to deal with the situation. The situation may involve your family, your job, your friends, or something else important to you. Please listen to each statement carefully and indicate how often you engaged in the following behaviors when you experienced a stressful situation since to coming to the United States. (CARD 14)

F1. I said prayers.

\begin{tabular}{|c|c|c|c|c|}
\hline Not used at all & Used sometimes & Used often & Used very often & Always \\
\hline 1 & 2 & 3 & 4 & 5 \\
\hline
\end{tabular}

F2. I read scriptures

\begin{tabular}{|c|c|c|c|c|}
\hline Not used at all & Used sometimes & Used often & Used very often & Always \\
\hline 1 & 2 & 3 & 4 & 5 \\
\hline
\end{tabular}

F3. I attended a religious support group.

\begin{tabular}{|c|c|c|c|c|}
\hline Not used at all & Used sometimes & Used often & Used very often & Always \\
\hline 1 & 2 & 3 & 4 & 5 \\
\hline
\end{tabular}

F4. Allowed the holy spirit to direct my actions

\begin{tabular}{|c|c|c|c|c|}
\hline Not used at all & Used sometimes & Used often & Used very often & Always \\
\hline 1 & 2 & 3 & 4 & 5 \\
\hline
\end{tabular}

F5. I confessed to God

\begin{tabular}{|c|c|c|c|c|}
\hline Not used at all & Used sometimes & Used often & Used very often & Always \\
\hline 1 & 2 & 3 & 4 & 5 \\
\hline
\end{tabular}

F6. I did not pray ${ }^{\circledR}$

\begin{tabular}{|c|c|c|c|c|}
\hline Not used at all & Used sometimes & Used often & Used very often & Always \\
\hline 1 & 2 & 3 & 4 & 5 \\
\hline
\end{tabular}

F7. I got support from church/mosque/temple leaders

\begin{tabular}{|c|c|c|c|c|}
\hline Not used at all & Used sometimes & Used often & Used very often & Always \\
\hline 1 & 2 & 3 & 4 & 5 \\
\hline
\end{tabular}


F8. I talked to church/mosque/temple leaders.

\begin{tabular}{|c|c|c|c|c|}
\hline Not used at all & Used sometimes & Used often & Used very often & Always \\
\hline 1 & 2 & 3 & 4 & 5 \\
\hline
\end{tabular}

F9. I looked for a lesson from God in the situation.

\begin{tabular}{|c|c|c|c|c|}
\hline Not used at all & Used sometimes & Used often & Used very often & Always \\
\hline 1 & 2 & 3 & 4 & 5 \\
\hline
\end{tabular}

F10. I tried to be a less sinful person.

\begin{tabular}{|c|c|c|c|c|}
\hline Not used at all & Used sometimes & Used often & Used very often & Always \\
\hline 1 & 2 & 3 & 4 & 5 \\
\hline
\end{tabular}

F11. I prayed to God for inspiration.

\begin{tabular}{|c|c|c|c|c|}
\hline Not used at all & Used sometimes & Used often & Used very often & Always \\
\hline 1 & 2 & 3 & 4 & 5 \\
\hline
\end{tabular}

F12. I tried to make up for my mistakes.

\begin{tabular}{|c|c|c|c|c|}
\hline Not used at all & Used sometimes & Used often & Used very often & Always \\
\hline 1 & 2 & 3 & 4 & 5 \\
\hline
\end{tabular}

F13. I put my problems in God's hands .

\begin{tabular}{|c|c|c|c|c|}
\hline Not used at all & Used sometimes & Used often & Used very often & Always \\
\hline 1 & 2 & 3 & 4 & 5 \\
\hline
\end{tabular}

F14. I prayed for strength.

\begin{tabular}{|c|c|c|c|c|}
\hline Not used at all & Used sometimes & Used often & Used very often & Always \\
\hline 1 & 2 & 3 & 4 & 5 \\
\hline
\end{tabular}

F15. I talked to church/mosque/temple members.

\begin{tabular}{|c|c|c|c|c|}
\hline Not used at all & Used sometimes & Used often & Used very often & Always \\
\hline 1 & 2 & 3 & 4 & 5 \\
\hline
\end{tabular}

F16. I counted my blessings.

\begin{tabular}{|c|c|c|c|c|}
\hline Not used at all & Used sometimes & Used often & Used very often & Always \\
\hline 1 & 2 & 3 & 4 & 5 \\
\hline
\end{tabular}

F17. I talked to my minister/preachers/rabbi/priest.

\begin{tabular}{|c|c|c|c|c|}
\hline Not used at all & Used sometimes & Used often & Used very often & Always \\
\hline 1 & 2 & 3 & 4 & 5 \\
\hline
\end{tabular}


F18. I recalled a Bible passage.

\begin{tabular}{|c|c|c|c|c|}
\hline Not used at all & Used sometimes & Used often & Used very often & Always \\
\hline 1 & 2 & 3 & 4 & 5 \\
\hline
\end{tabular}

F19. I stopped going to religious services. ${ }^{\circledR}$

\begin{tabular}{|c|c|c|c|c|}
\hline Not used at all & Used sometimes & Used often & Used very often & Always \\
\hline 1 & 2 & 3 & 4 & 5 \\
\hline
\end{tabular}

F20. I got help from clergy

\begin{tabular}{|c|c|c|c|c|}
\hline Not used at all & Used sometimes & Used often & Used very often & Always \\
\hline 1 & 2 & 3 & 4 & 5 \\
\hline
\end{tabular}

F21. I used a Bible story to help solve a problem.

\begin{tabular}{|c|c|c|c|c|}
\hline Not used at all & Used sometimes & Used often & Used very often & Always \\
\hline 1 & 2 & 3 & 4 & 5 \\
\hline
\end{tabular}

F22. I prayed for the help of a religious figure.

\begin{tabular}{|c|c|c|c|c|}
\hline Not used at all & Used sometimes & Used often & Used very often & Always \\
\hline 1 & 2 & 3 & 4 & 5 \\
\hline
\end{tabular}

F23. I solved problems without God's help. ${ }^{\circledR}$

\begin{tabular}{|c|c|c|c|c|}
\hline Not used at all & Used sometimes & Used often & Used very often & Always \\
\hline 1 & 2 & 3 & 4 & 5 \\
\hline
\end{tabular}

F24. I asked for God's forgiveness.

\begin{tabular}{|c|c|c|c|c|}
\hline Not used at all & Used sometimes & Used often & Used very often & Always \\
\hline 1 & 2 & 3 & 4 & 5 \\
\hline
\end{tabular}

F25. I donated time to a religious cause or activity.

\begin{tabular}{|c|c|c|c|c|}
\hline Not used at all & Used sometimes & Used often & Used very often & Always \\
\hline 1 & 2 & 3 & 4 & 5 \\
\hline
\end{tabular}

F26. I asked my religious leader for advice.

\begin{tabular}{|c|c|c|c|c|}
\hline Not used at all & Used sometimes & Used often & Used very often & Always \\
\hline 1 & 2 & 3 & 4 & 5 \\
\hline
\end{tabular}

F27. I shared my religious beliefs with others.

\begin{tabular}{|c|c|c|c|c|}
\hline Not used at al & Used sometimes & Used often & Used very often & Always \\
\hline 1 & 2 & 3 & 4 & 5 \\
\hline
\end{tabular}


F28. I thought about Jesus (God) as my friend.

\begin{tabular}{|c|c|c|c|c|}
\hline Not used at all & Used sometimes & Used often & Used very often & Always \\
\hline 1 & 2 & 3 & 4 & 5 \\
\hline
\end{tabular}

F29. I got involved with $\mathrm{church} / \mathrm{mosque} / \mathrm{temple}$ activities.

\begin{tabular}{|c|c|c|c|c|}
\hline Not used at all & Used sometimes & Used often & Used very often & Always \\
\hline 1 & 2 & 3 & 4 & 5 \\
\hline
\end{tabular}

F30. I gave money to a religious organization.

\begin{tabular}{|c|c|c|c|c|}
\hline Not used at all & Used sometimes & Used often & Used very often & Always \\
\hline 1 & 2 & 3 & 4 & 5 \\
\hline
\end{tabular}

F31. I based life decisions on my religious beliefs.

\begin{tabular}{|c|c|c|c|c|}
\hline Not used at all & Used sometimes & Used often & Used very often & Always \\
\hline 1 & 2 & 3 & 4 & 5 \\
\hline
\end{tabular}

F32. I found peace by going to a religious place.

\begin{tabular}{|c|c|c|c|c|}
\hline Not used at all & Used sometimes & Used often & Used very often & Always \\
\hline 1 & 2 & 3 & 4 & 5 \\
\hline
\end{tabular}

F33. I asked someone to pray for me.

\begin{tabular}{|c|c|c|c|c|}
\hline Not used at all & Used sometimes & Used often & Used very often & Always \\
\hline 1 & 2 & 3 & 4 & 5 \\
\hline
\end{tabular}

F34. I asked for blessings.

\begin{tabular}{|c|c|c|c|c|}
\hline Not used at all & Used sometimes & Used often & Used very often & Always \\
\hline 1 & 2 & 3 & 4 & 5 \\
\hline
\end{tabular}

F35. I prayed for help.

\begin{tabular}{|c|c|c|c|c|}
\hline Not used at all & Used sometimes & Used often & Used very often & Always \\
\hline 1 & 2 & 3 & 4 & 5 \\
\hline
\end{tabular}

F36. I went to a religious counselor.

\begin{tabular}{|c|c|c|c|c|}
\hline Not used at all & Used sometimes & Used often & Used very often & Always \\
\hline 1 & 2 & 3 & 4 & 5 \\
\hline
\end{tabular}


F37. I worked with God to solve problems.

\begin{tabular}{|c|c|c|c|c|}
\hline Not used at all & Used sometimes & Used often & Used very often & Always \\
\hline 1 & 2 & 3 & 4 & 5 \\
\hline
\end{tabular}

F38. I found peace by sharing my problems with God.

\begin{tabular}{|c|c|c|c|c|}
\hline Not used at all & Used sometimes & Used often & Used very often & Always \\
\hline 1 & 2 & 3 & 4 & 5 \\
\hline
\end{tabular}

F39. I stopped reading scripture. ${ }^{\circledR}$

\begin{tabular}{|c|c|c|c|c|}
\hline Not used at all & Used sometimes & Used often & Used very often & Always \\
\hline 1 & 2 & 3 & 4 & 5 \\
\hline
\end{tabular}

F40. I recite a psalm.

\begin{tabular}{|c|c|c|c|c|}
\hline Not used at all & Used sometimes & Used often & Used very often & Always \\
\hline 1 & 2 & 3 & 4 & 5 \\
\hline
\end{tabular}




\section{Appendix $\mathbf{J}$}

\section{Ways of Religious Coping Scale (WORSC Spanish Version)}

Las siguientes preguntas están relacionadas con como usted a manejado situaciones estresantes en su vida desde llegar a los Estados Unidos. Una situación "estresante" es cualquier situación que es dificil o preocupante para usted, porque usted se sentía angustiado sobre lo que pasaba o porque usted tenía que usar un esfuerzo considerable para lidiar con la situación. La situación puede implicar su familia, su trabajo, sus amigos, o algo más importante para usted. Por favor escuche cada frase con cuidado e indique con qué frecuencia usted tuvo los siguientes comportamientos cuando estuvo en una situación estresante desde su inmigración a los Estados Unidos. Cuando decimos "Dios" en las próximas preguntas nos referimos a un ser supremo. (TARJETA 14)

F1. Recé.

\begin{tabular}{|c|c|c|c|c|}
\hline Nunca & Algunas veces & A menudo & Muy a menudo & Siempre \\
\hline 1 & 2 & 3 & 4 & 5 \\
\hline
\end{tabular}

F2. Leí escrituras religiosas

\begin{tabular}{|c|c|c|c|c|}
\hline Nunca & Algunas veces & A menudo & Muy a menudo & Siempre \\
\hline 1 & 2 & 3 & 4 & 5 \\
\hline
\end{tabular}

F3. Asistí a un grupo de apoyo religioso.

\begin{tabular}{|c|c|c|c|c|}
\hline Nunca & Algunas veces & A menudo & Muy a menudo & Siempre \\
\hline 1 & 2 & 3 & 4 & 5 \\
\hline
\end{tabular}

F4. Permití que un ser supremo dirija mis acciones

\begin{tabular}{|c|c|c|c|c|}
\hline Nunca & Algunas veces & A menudo & Muy a menudo & Siempre \\
\hline 1 & 2 & 3 & 4 & 5 \\
\hline
\end{tabular}

F5. Yo me confesé con Dios

\begin{tabular}{|c|c|c|c|c|}
\hline Nunca & Algunas veces & A menudo & Muy a menudo & Siempre \\
\hline 1 & 2 & 3 & 4 & 5 \\
\hline
\end{tabular}

F6. No recé ${ }^{\circledR}$

\begin{tabular}{|c|c|c|c|c|}
\hline Nunca & Algunas veces & A menudo & Muy a menudo & Siempre \\
\hline 1 & 2 & 3 & 4 & 5 \\
\hline
\end{tabular}

F7. Conseguí el apoyo de líderes de la iglesia/mezquita/templo/ o espirituales

\begin{tabular}{|c|c|c|c|c|}
\hline Nunca & Algunas veces & A menudo & Muy a menudo & Siempre \\
\hline 1 & 2 & 3 & 4 & 5 \\
\hline
\end{tabular}


F8. Hable con líderes de la iglesia/mezquita/templo/ o espirituales.

\begin{tabular}{|c|c|c|c|c|}
\hline Nunca & Algunas veces & A menudo & Muy a menudo & Siempre \\
\hline 1 & 2 & 3 & 4 & 5 \\
\hline
\end{tabular}

F9. Busqué una lección de Dios en la situación.

\begin{tabular}{|c|c|c|c|c|}
\hline Nunca & Algunas veces & A menudo & Muy a menudo & Siempre \\
\hline 1 & 2 & 3 & 4 & 5 \\
\hline
\end{tabular}

F10. Traté de ser una persona menos pecadora.

\begin{tabular}{|c|c|c|c|c|}
\hline Nunca & Algunas veces & A menudo & Muy a menudo & Siempre \\
\hline 1 & 2 & 3 & 4 & 5 \\
\hline
\end{tabular}

F11. Le recé a Dios por inspiración.

\begin{tabular}{|c|c|c|c|c|}
\hline Nunca & Algunas veces & A menudo & Muy a menudo & Siempre \\
\hline 1 & 2 & 3 & 4 & 5 \\
\hline
\end{tabular}

F12. Traté de compensar mis errores.

\begin{tabular}{|c|c|c|c|c|}
\hline Nunca & Algunas veces & A menudo & Muy a menudo & Siempre \\
\hline 1 & 2 & 3 & 4 & 5 \\
\hline
\end{tabular}

F13. Puse mis problemas en las manos de Dios.

\begin{tabular}{|c|c|c|c|c|}
\hline Nunca & Algunas veces & A menudo & Muy a menudo & Siempre \\
\hline 1 & 2 & 3 & 4 & 5 \\
\hline
\end{tabular}

F14. Recé por fuerza.

\begin{tabular}{|c|c|c|c|c|}
\hline Nunca & Algunas veces & A menudo & Muy a menudo & Siempre \\
\hline 1 & 2 & 3 & 4 & 5 \\
\hline
\end{tabular}

F15. Hable con miembros de iglesia/mezquita/templo.

\begin{tabular}{|c|c|c|c|c|}
\hline Nunca & Algunas veces & A menudo & Muy a menudo & Siempre \\
\hline 1 & 2 & 3 & 4 & 5 \\
\hline
\end{tabular}

F16. Conté mis bendiciones.

\begin{tabular}{|c|c|c|c|c|}
\hline Nunca & Algunas veces & A menudo & Muy a menudo & Siempre \\
\hline 1 & 2 & 3 & 4 & 5 \\
\hline
\end{tabular}

F17. Hable con mi ministro/predicadores/rabino/sacerdote/u otro líder religioso.

\begin{tabular}{|c|c|c|c|c|}
\hline Nunca & Algunas veces & A menudo & Muy a menudo & Siempre \\
\hline 1 & 2 & 3 & 4 & 5 \\
\hline
\end{tabular}


F18. Recordé un verso de la Biblia u otra escritura religiosa.

\begin{tabular}{|c|c|c|c|c|}
\hline Nunca & Algunas veces & A menudo & Muy a menudo & Siempre \\
\hline 1 & 2 & 3 & 4 & 5 \\
\hline
\end{tabular}

F19. Dejé de ir a servicios religiosos. ${ }^{\circledR}$

\begin{tabular}{|c|c|c|c|c|}
\hline Nunca & Algunas veces & A menudo & Muy a menudo & Siempre \\
\hline 1 & 2 & 3 & 4 & 5 \\
\hline
\end{tabular}

F20. Conseguí la ayuda del clero u otro líder religioso

\begin{tabular}{|c|c|c|c|c|}
\hline Nunca & Algunas veces & A menudo & Muy a menudo & Siempre \\
\hline 1 & 2 & 3 & 4 & 5 \\
\hline
\end{tabular}

F21. Usé una historia de la Biblia u otra escritura religiosa para ayudarme a solucionar un problema.

\begin{tabular}{|c|c|c|c|c|}
\hline Nunca & Algunas veces & A menudo & Muy a menudo & Siempre \\
\hline 1 & 2 & 3 & 4 & 5 \\
\hline
\end{tabular}

F22. Recé para la ayuda de una figura religiosa.

\begin{tabular}{|c|c|c|c|c|}
\hline Nunca & Algunas veces & A menudo & Muy a menudo & Siempre \\
\hline 1 & 2 & 3 & 4 & 5 \\
\hline
\end{tabular}

F23. Solucioné problemas sin la ayuda de Dios. ${ }^{\circledR}$

\begin{tabular}{|c|c|c|c|c|}
\hline Nunca & Algunas veces & A menudo & Muy a menudo & Siempre \\
\hline 1 & 2 & 3 & 4 & 5 \\
\hline
\end{tabular}

F24. Pedí el perdón de Dios.

\begin{tabular}{|c|c|c|c|c|}
\hline Nunca & Algunas veces & A menudo & Muy a menudo & Siempre \\
\hline 1 & 2 & 3 & 4 & 5 \\
\hline
\end{tabular}

F25. Doné de mi tiempo a una causa religiosa.

\begin{tabular}{|c|c|c|c|c|}
\hline Nunca & Algunas veces & A menudo & Muy a menudo & Siempre \\
\hline 1 & 2 & 3 & 4 & 5 \\
\hline
\end{tabular}

F26. Le pedí consejos a mi líder religioso.

\begin{tabular}{|c|c|c|c|c|}
\hline Nunca & Algunas veces & A menudo & Muy a menudo & Siempre \\
\hline 1 & 2 & 3 & 4 & 5 \\
\hline
\end{tabular}

F27. Compartí mis creencias religiosas con otros.

\begin{tabular}{|c|c|c|c|c|}
\hline Nunca & Algunas veces & A menudo & Muy a menudo & Siempre \\
\hline 1 & 2 & 3 & 4 & 5 \\
\hline
\end{tabular}


F28. Pensé en Jesús (Dios) u otro ser supremo como mi amigo.

\begin{tabular}{|c|c|c|c|c|}
\hline Nunca & Algunas veces & A menudo & Muy a menudo & Siempre \\
\hline 1 & 2 & 3 & 4 & 5 \\
\hline
\end{tabular}

F29. Estuve involucrado con actividades religiosas como las de iglesia/mezquita/templo.

\begin{tabular}{|c|c|c|c|c|}
\hline Nunca & Algunas veces & A menudo & Muy a menudo & Siempre \\
\hline 1 & 2 & 3 & 4 & 5 \\
\hline
\end{tabular}

F30. Le di dinero a una organización religiosa.

\begin{tabular}{|c|c|c|c|c|}
\hline Nunca & Algunas veces & A menudo & Muy a menudo & Siempre \\
\hline 1 & 2 & 3 & 4 & 5 \\
\hline
\end{tabular}

F31. Base las decisiones de la vida en mis creencias religiosas.

\begin{tabular}{|c|c|c|c|c|}
\hline Nunca & Algunas veces & A menudo & Muy a menudo & Siempre \\
\hline 1 & 2 & 3 & 4 & 5 \\
\hline
\end{tabular}

F32. Encontré la paz yendo a un lugar religioso.

\begin{tabular}{|c|c|c|c|c|}
\hline Nunca & Algunas veces & A menudo & Muy a menudo & Siempre \\
\hline 1 & 2 & 3 & 4 & 5 \\
\hline
\end{tabular}

F33. Le pedí a alguien que rezara por mí.

\begin{tabular}{|c|c|c|c|c|}
\hline Nunca & Algunas veces & A menudo & Muy a menudo & Siempre \\
\hline 1 & 2 & 3 & 4 & 5 \\
\hline
\end{tabular}

F34. Pedí por bendiciones.

\begin{tabular}{|c|c|c|c|c|}
\hline Nunca & Algunas veces & A menudo & Muy a menudo & Siempre \\
\hline 1 & 2 & 3 & 4 & 5 \\
\hline
\end{tabular}

F35. Recé por ayuda.

\begin{tabular}{|c|c|c|c|c|}
\hline Nunca & Algunas veces & A menudo & Muy a menudo & Siempre \\
\hline 1 & 2 & 3 & 4 & 5 \\
\hline
\end{tabular}

F36. Fui a un consejero religioso.

\begin{tabular}{|c|c|c|c|c|}
\hline Nunca & Algunas veces & A menudo & Muy a menudo & Siempre \\
\hline 1 & 2 & 3 & 4 & 5 \\
\hline
\end{tabular}

F37. Trabajé con Dios para solucionar problemas.

\begin{tabular}{|c|c|c|c|c|}
\hline Nunca & Algunas veces & A menudo & Muy a menudo & Siempre \\
\hline 1 & 2 & 3 & 4 & 5 \\
\hline
\end{tabular}

F38. Encontré la paz compartiendo mis problemas con Dios.

\begin{tabular}{|c|c|c|c|c|}
\hline Nunca & Algunas veces & A menudo & Muy a menudo & Siempre \\
\hline 1 & 2 & 3 & 4 & 5 \\
\hline
\end{tabular}


F39. Dejé de leer las escrituras religiosas. ${ }^{\circledR}$

\begin{tabular}{|c|c|c|c|c|}
\hline Nunca & Algunas veces & A menudo & Muy a menudo & Siempre \\
\hline 1 & 2 & 3 & 4 & 5 \\
\hline
\end{tabular}

F40. Recite un salmo u otra escritura religiosa.

\begin{tabular}{|c|c|c|c|c|}
\hline Nunca & Algunas veces & A menudo & Muy a menudo & Siempre \\
\hline 1 & 2 & 3 & 4 & 5 \\
\hline
\end{tabular}




\section{Appendix K}

\section{The Brief RCOPE}

The following items deal with ways you coped with negative life events since you have been in the United States. There are many ways to try to deal with problems. These items ask what you did to cope with these negative life events. Obviously different people deal with things in different ways. Each item says something about a particular way of coping. We want to know to what extent you did what the item says. How much or frequently. Don't answer on the basis of what worked or not just whether you did it. Use these response choices. Try to rate each item separately in your mind from the others. Make your answers as true for you as you can.

1. Looked for a stronger connection with God.
$1-$ not at all
2-somewhat
3-quite a bit
4-a great deal

2. Sought God's love and care.

1-not at all 2-somewhat 3-quite a bit 4-a great deal

3. Sought help from God in letting go.

1-not at all 2-somewhat 3-quite a bit 4-a great deal

4. Tried to put my plans into action together with God.

1-not at all 2-somewhat 3-quite a bit 4-a great deal

5. Tried to see how God might be trying to strengthen me in the situation.

1-not at all 2-somewhat 3-quite a bit 4-a great deal

6. Asked for forgiveness for my sins.
$1-$ not at all
2-somewhat
3-quite a bit
4-a great deal 
7. Focused on religion to stop worrying about my problems.

1-not at all 2-somewhat 3-quite a bit $\quad$ 4-a great deal

8. Wondered whether God had abandoned me.

1-not at all 2-somewhat 3-quite a bit $\quad$ 4-a great deal

9. Felt punished by God for my lack of devotion

1-not at all 2-somewhat 3-quite a bit $\quad$ 4-a great deal

10. Wondered what I did for God to punish me.

1-not at all 2-somewhat $\quad 3$-quite a bit $\quad$ 4-a great deal

11. Questioned God' love for me.

1-not at all 2-somewhat $\quad 3$-quite a bit $\quad$ 4-a great deal

12. Wondered whether my church had abandoned me.

1-not at all 2-somewhat $\quad 3$-quite a bit $\quad$ 4-a great deal

13. Decided the devil made this happened

1-not at all 2-somewhat 3-quite a bit $\quad$ 4-a great deal

14. Questioned the power of God

1-not at all 2-somewhat 3-quite a bit $\quad$ 4-a great deal 


\section{Appendix L}

\section{The Brief RCOPE (Spanish Version)}

Las siguientes frases tratan con maneras en que usted se ajusto o supero eventos negativos en su vida desde su inmigración a los Estados Unidos. Hay muchas maneras de cómo superar problemas. Estas frases preguntan que hizo usted para superar estos eventos negativos de su vida. Obviamente cada persona supera cosas en maneras diferentes, pero nos interesa saber como usted intento de superarlas. Cada frase dice algo sobre una manera particular de superar situaciones. Nosotros queremos saber que tanto hizo usted lo que indica cada frase. Cuanto o con que frecuencia. No responda según a lo que le funciono o no, solamente responda si lo hizo o no. Use las respuestas incluidas. Trate de responder en su mente cada pregunta independientemente de las otras. Responda a cada pregunta lo mas cierto que sea para usted. Cuando decimos "Dios" en las próximas preguntas nos referimos a un ser supremo.

1. Busque una conexión más fuerte con Dios.
1-nunca
2-de vez en cuando
3-mucho
4-muchisimo

2. Busque el amor y el cuidado de Dios.
1-nunca
2-de vez en cuando
3-mucho
4-muchisimo

3. Busque ayuda de Dios para dejar ir mi coraje.
1-nunca
2-de vez en cuando
3-mucho
4-muchisimo

4. Trate de poner mis planes en acción junto con Dios.
1-nunca
2-de vez en cuando
3-mucho
4-muchisimo

5. Trate de ver como Dios podría estar tratando de fortalecerme en la situación.

1-nunca 2-de vez en cuando 3-mucho 4-muchisimo

6. Pedí perdón por mis pecados.

1-nunca 2-de vez en cuando 3-mucho 4-muchisimo

7. Me enfoque en la religión para dejar de preocuparme en mis problemas.

1-nunca 2-de vez en cuando 3-mucho 4-muchisimo 
8. Me pregunte si Dios me había abandonado.

1-nunca 2-de vez en cuando 3-mucho 4-muchisimo

9. Me sentí castigado por Dios por mi falta de devoción.

1-nunca 2-de vez en cuando 3-mucho 4-muchisimo

10. Me pregunte que había hecho yo para que Dios

1-nunca 2-de vez en cuando 3-mucho 4-muchisimo

11. Cuestione el amor de Dios hacia me.

1-nunca 2-de vez en cuando 3-mucho 4-muchisimo

12. Me pregunte si mi Iglesia me había abandonado.

1-nunca 2-de vez en cuando 3-mucho 4-muchisimo

13. Decidí que el diablo hizo que esto pasara.

1-nunca 2-de vez en cuando 3-mucho 4-muchisimo

14. Cuestione el poder de Dios.

1-nunca 2-de vez en cuando 3-mucho 4-muchisimo 
VITA

\section{MARIANA SANCHEZ}

1998

2003

$2008-2012$
B.A., Psychology

Florida International University

Miami, Florida

M.S.W, Social Work

Florida International University

Miami, Florida

Doctoral Candidate in Social Welfare

Florida International University

Miami, Florida

\section{PUBLICATIONS AND PRESENTATIONS}

De La Rosa, M., Sanchez, M., Dillon, F., Ruffin, B.A., Blackson, T., \& Schwartz. (2011). Alcohol use among Latinos: A comparison of pre-immigration, post-immigration, and U.S. born Latinos. Journal of Immigrant and Minority Health. doi: 10.1007/s10903-0119498-x

Dillon, F.R., De La Rosa, M.R., \& Sanchez, M., \& Schwartz, S. (in press). Preimmigration family cohesion and drug/alcohol abuse recent Latino immigrants. The Family Journal.

De la Rosa, M., Rojas, P., Sanchez, M. (in press) A Case Study in Advocating for Health Care Change at the Community level: The Community Voices Health care Initiative. In R. Braithwaite Community Voices: Health Matters. Jossey Bass Publishers, San Francisco, CA.

Sanchez, M., Dillon, F.R., Ruffin, B.A., \& De La Rosa, M. (2011, October). Examining the Relationship between Positive/Negative Religious Coping and Acculturative Stress among Recent Latino Immigrants. Poster presented at the Annual Meeting for the American Public Health Association. Washington D.C. October 2011.

De La Rosa, M. \& Sanchez, M. (2011, April). The Recent Latino Immigrant Study: Examining the influence of pre-immigration assets on the substance use and HIV-risk behaviors of recent Latino immigrants. Paper presented at the $\mathrm{Bi}$-Annual Conference of the Center for Substance Use \& HIV/AIDS Research on Latinos in the United States.. North Miami, FL. April 2011.

De La Rosa, M., Sanchez, M., Ruffin, B., \& Dillon, F. (2011, March). Alcohol use among Latino adults: A comparison of pre-immigration, post-immigration, and U.S. born 
Latinos. Paper presented at Florida International University Social Work Scholars Colloquium Series. Miami, FL.

Sanchez, M., Dillon, F., Ruffin, B. \& De La Rosa, M. (2011, January). The influence of religious coping on the acculturative stress of recent Latino immigrants. Poster presented at Society for Social Work Research Annual Conference. Tampa, FL. January 2011.

Sanchez, M., Rojas, P., Dillon, F. \& De La Rosa, M. (2010, January). Religiosity as a Cultural Protective Factor against Illicit Drug Use among Latina Women. Poster presented at the Annual Meeting for the Society for Social Work Research. San Francisco, CA. January 2010. 